ESAIM: M2AN 53 (2019) 1-34

https://doi.org/10.1051/m2an/2019002
ESAIM: Mathematical Modelling and Numerical Analysis

www.esaim-m2an.org

\title{
REPRESENTATION OF CAPACITY DROP AT A ROAD MERGE VIA POINT CONSTRAINTS IN A FIRST ORDER TRAFFIC MODEL
}

\author{
Edda Dal Santo ${ }^{1}$, Carlotta Donadello $^{2, *}$, Sabrina F. Pellegrino ${ }^{3}$, \\ and Massimiliano D. Rosini ${ }^{4,5}$
}

\begin{abstract}
We reproduce the capacity drop phenomenon at a road merge by implementing a non-local point constraint at the junction in a first order traffic model. We call capacity drop the situation in which the outflow through the junction is lower than the receiving capacity of the outgoing road, as too many vehicles trying to access the junction from the incoming roads hinder each other. In this paper, we first construct an enhanced version of the locally constrained model introduced by Haut et al. (Proceedings 16th IFAC World Congress. Prague, Czech Republic 229 (2005) TuM01TP/3), then we propose its counterpart featuring a non-local constraint and finally we compare numerically the two models by constructing an adapted finite volumes scheme.
\end{abstract}

Mathematics Subject Classification. 35L65, 35R02, 90B20, 76M12.

Received March 12, 2018. Accepted December 28, 2018.

\section{INTRODUCTION AND MAIN RESULTS}

In macroscopic vehicular traffic modeling a particular attention is devoted to the dynamics of crossroads, as it is the essential building block to the modeling of traffic in a road network. From the mathematical point of view, the basic model for a crossroad is given by a system of conservation laws on an oriented star shaped graph. In this setting, the mere assumption of conservation of the number of vehicles through the junction is not enough to ensure the uniqueness of solutions. Further conditions, depending on the specific situation we aim at describing, should be imposed. This explains the huge number of such models available in the literature, see for instance $[8,12,19-22]$ and the references therein.

The aim of this paper is to introduce and compare simple first order models able to reproduce the capacity drop phenomenon at a merge. We call capacity drop the situation in which the outflow through the junction is

Keywords and phrases. Scalar conservation law, LWR model, traffic flow on networks, point constraint on the flux, finite volumes schemes.

1 Dipartimento di Ingegneria e Scienze de l'Informazione e Matematica, Università dell'Aquila, Via Vetoio, 67100 L'Aquila, Italy.

2 Laboratoire de mathématiques, CNRS UMR 6623, Université de Bourgogne Franche-Comté, 16 route de Gray, 25030 Besançon, France.

3 Dipartimento di Matematica, Università di Bari, Via E. Orabona 4, 70126 Bari, Italy.

4 Dipartimento di Matematica e Informatica, Università di Ferrara, Via Machiavelli 35, 44121 Ferrara, Italy.

5 Uniwersytet Marii Curie-Skłodowskiej, Plac Marii Curie-Skłodowskiej 1, 20-031 Lublin, Poland.

*Corresponding author: carlotta.donadello@univ-f comte.fr 
lower than the receiving capacity of the outgoing road, as too many vehicles trying to access the junction from the incoming roads hinder each other.

To the best of our knowledge, the first model aiming to capture such phenomenon is the Haut-Bastin-Chitour (HBC) model introduced in [22]. Roughly speaking, the authors introduce a decreasing function of the sending capacity of the incoming roads to bound the receiving capacity of the outgoing one. This model suffers two drawbacks: from the modeling point of view, it can produce a persistent traffic jam even starting from initial conditions which lead to moderate transient congestion in the unconstrained model; from the mathematical point of view, this model is not consistent, see Definition 2.6. In the present paper, we first construct an enhanced version of the HBC model, then we propose a new model in which the capacity drop is reproduced by imposing a non-local point constraint at the junction, and finally compare numerically the two models.

The non-local model can be seen as the natural generalization to the framework of a merge of the approach used in [2-5], where the authors developed analytical and numerical tools for the representation of capacity drop in vehicular and pedestrian traffic models on a single road by means of non-local point constraints on the flux. One of the main advantages of using non-local point constraints instead of local point constraints, as it was done in [9-11,13-17], is that the non-local approach allows for a more realistic representation of the transient behavior between congested traffic and free flow, see for instance [3] where the model presented in [16] is compared with its non-local counterpart. We stress that the introduction of a non-local point constraint in the model does not substitute the implementation of a ramp metering strategy to avoid the formation of a traffic jam, as the description of the traffic evolution and its control are of course separate issues.

We construct a finite volumes scheme by adapting the finite volumes method introduced in [1] to the constrained case, similarly to what has been done in [3]. We validate our scheme and implementation for both the local and non-local constraint case by comparison with explicit solutions here computed. Our simulations show that, at least in these cases, the method converges numerically and that the implementation of a non-local point constraint allows for a more regular transition between congested and free traffic situations. Moreover, a qualitative analysis of the behavior of numerical solutions suggests a simple way to calibrate the parameters appearing in our non-local operator.

The paper is organized as follows. The next section is an introduction to the basic definitions and notations used in the paper. In Section 3 we outline the main features of the local model. In Section 4 we briefly comment on the properties of the non-local model. Section 5 is devoted to the description and validation of the adapted finite volumes numerical scheme. Section 5.3 presents a numerical comparison of local and non-local models. Section 6 contains lengthy computations and technical proofs. The last section is devoted to concluding remarks.

\section{BASIC DEFINITIONS AND NOTATIONS}

In this paper we consider a junction (crossroad) connecting two incoming and one outgoing roads. In terms of graph theory, we consider a semi-infinite star-graph with two incoming and one outgoing edges.

The incoming roads are parameterized by $x \in(-\infty, 0]$ and numbered by the index $i \in \mathrm{I}=\{1,2\}$, while the outgoing road is parameterized by $x \in[0, \infty)$ and numbered by the index 3 . In both parameterizations the junction is located at $x=0$. We denote the generic road by $\Omega_{h}, h \in \mathrm{H}=\{1,2,3\}$, and the network by $\mathcal{N}=\Pi_{h \in \mathrm{H}} \Omega_{h}$.

On each road the traffic evolution is described by the Lighthill-Whitham-Richards (LWR) model [24, 27], namely by a scalar conservation law of the form

$$
\partial_{t} \rho_{h}+\partial_{x} f_{h}\left(\rho_{h}\right)=0, \quad t>0, x \in \Omega_{h},
$$

where $\rho_{h}$ is the density of vehicles and $f_{h}$ is the flux along the $h$ th road. We augment $(2.1)$ with the initial condition

$$
\rho_{h}(0, x)=\rho_{h, 0}(x), \quad x \in \Omega_{h},
$$


where $\rho_{h, 0}$ is assumed to be in $\mathrm{L}^{1} \cap \mathrm{BV}\left(\Omega_{h} ;\left[0, \rho_{\max }\right]\right)$. We assume that the roads have a common maximal density $\rho_{\max }>0$ and the fluxes $f_{h}$ are bell-shaped (unimodal). More precisely we assume that

$f_{h}$ belongs to $\operatorname{Lip}\left(\left[0, \rho_{\max }\right] ;\left[0, f_{h}^{\max }\right]\right), f_{h}(0)=0=f_{h}\left(\rho_{\max }\right)$ and there exists $\rho_{h, c} \in\left(0, \rho_{\max }\right)$ such that $f_{h}^{\prime}(\rho)\left(\rho_{h, c}-\rho\right)>0$ for a.e. $\rho \in\left[0, \rho_{\max }\right]$.

Above $f_{h}^{\max }$ is the maximum value of $f_{h}$ in $\left[0, \rho_{\max }\right]$ and we have $f_{h}^{\max }=f_{h}\left(\rho_{h, c}\right)$. Assumption $(\mathrm{F})$ is compatible with the standard LWR traffic model, see $[8,19,20,28]$, and appears in most papers devoted to conservation laws subject to point constraints $[1,4,6,9,13]$ as it ensures the existence of strong traces of solutions on both sides of the constraint location, see Theorem 2.2 of [6] which is a reformulation of the results of $[25,29]$. The same hypothesis has also been used in [22], which is the starting point of our analysis.

Let $\bar{\rho}_{h}$ be the trace of $\rho_{h}$ at $x=0, h \in \mathrm{H}$, and $\gamma(\vec{\rho})=\left(\bar{\rho}_{1}, \bar{\rho}_{2}, \bar{\rho}_{3}\right)$ the vector of the traces of the densities at the junction. We use the following definition of weak solution on the network.

Definition 2.1. The vector function $\vec{\rho}=\left(\rho_{1}, \rho_{2}, \rho_{3}\right)$, where $\rho_{h}:(0, \infty) \times \Omega_{h} \rightarrow\left[0, \rho_{\max }\right], h \in \mathbf{H}$, is a weak solution to (2.1) and (2.2), $h \in \mathrm{H}$, in the network $\mathcal{N}$ if

$-\rho_{h} \in \mathrm{C}^{0}\left((0, \infty) ; \mathrm{L}^{1}\left(\Omega_{h} ;\left[0, \rho_{\text {max }}\right]\right)\right) \cap \mathrm{BV}_{\text {loc }}\left((0, \infty) \times \Omega_{h} ;\left[0, \rho_{\text {max }}\right]\right), h \in \mathbf{H}$;

- for $i \in \mathrm{I}, \rho_{i}$ is a weak entropy solution to $(2.1)$ and $(2.2)_{h=i}$, namely for every $c \in\left[0, \rho_{\text {max }}\right]$ and every nonnegative test function $\phi \in \mathrm{C}^{\infty}(\mathbb{R} \times(-\infty, 0) ; \mathbb{R})$ with compact support

$$
\int_{0}^{\infty} \int_{\Omega_{i}}\left(\left|\rho_{i}-c\right| \partial_{t} \phi+\operatorname{sign}\left(\rho_{i}-c\right)\left(f_{i}\left(\rho_{i}\right)-f_{i}(c)\right) \partial_{x} \phi\right) \mathrm{d} x \mathrm{~d} t+\int_{\Omega_{i}}\left|\rho_{i, 0}(x)-c\right| \phi(0, x) \mathrm{d} x \geq 0
$$

- $\rho_{3}$ is a weak entropy solution to $(2.1)$ and $(2.2)_{h=3}$, namely for every $c \in\left[0, \rho_{\max }\right]$ and every nonnegative test function $\phi \in \mathrm{C}^{\infty}(\mathbb{R} \times(0, \infty) ; \mathbb{R})$ with compact support

$$
\int_{0}^{\infty} \int_{\Omega_{3}}\left(\left|\rho_{3}-c\right| \partial_{t} \phi+\operatorname{sign}\left(\rho_{3}-c\right)\left(f_{3}\left(\rho_{3}\right)-f_{3}(c)\right) \partial_{x} \phi\right) \mathrm{d} x \mathrm{~d} t+\int_{\Omega_{3}}\left|\rho_{3,0}(x)-c\right| \phi(0, x) \mathrm{d} x \geq 0
$$

- the number of vehicles across the junction is conserved, namely

$$
f_{3}\left(\bar{\rho}_{3}(t)\right)=f_{1}\left(\bar{\rho}_{1}(t)\right)+f_{2}\left(\bar{\rho}_{2}(t)\right), \quad \text { for a.e. } t>0 .
$$

Since the above definition does not ensure uniqueness of weak solutions, we need some additional selection criteria in order to hope for well-posedness of the Cauchy problem. This is achieved by declaring which Riemann solver we adopt at the junction, see $[8,12,19-22]$. We recall the following definition of Riemann solver at the junction.

Definition 2.2. We say that

$$
\mathcal{R S}=\left(\mathcal{R S}_{1}, \mathcal{R S}_{2}, \mathcal{R S}_{3}\right): \Lambda \rightarrow \mathrm{BV}(\mathcal{N} ; \Lambda), \quad \Lambda=\left[0, \rho_{\max }\right]^{3},
$$

is a Riemann solver at the junction if for any constant initial datum $\vec{\rho}_{0}=\left(\rho_{1,0}, \rho_{2,0}, \rho_{3,0}\right) \in \Lambda$ the map

$$
(t, \vec{x}) \mapsto \mathcal{R S}\left[\vec{\rho}_{0}\right](\vec{x} / t)=\left(\mathcal{R} \mathcal{S}_{1}\left[\vec{\rho}_{0}\right]\left(x_{1} / t\right), \mathcal{R S}_{2}\left[\vec{\rho}_{0}\right]\left(x_{2} / t\right), \mathcal{R} \mathcal{S}_{3}\left[\vec{\rho}_{0}\right]\left(x_{3} / t\right)\right)
$$

is a self-similar weak solution to $(2.1)$ and $(2.2), h \in \mathrm{H}$, in the network $\mathcal{N}$.

In Definition 2.6 below we state some natural properties for Riemann solvers at the junction. In order to do so, we have to recall some notation from the literature on traffic modeling.

Definition 2.3 (Def. 2.6 in [8]). We distinguish between good and bad (initial) data as follows: 

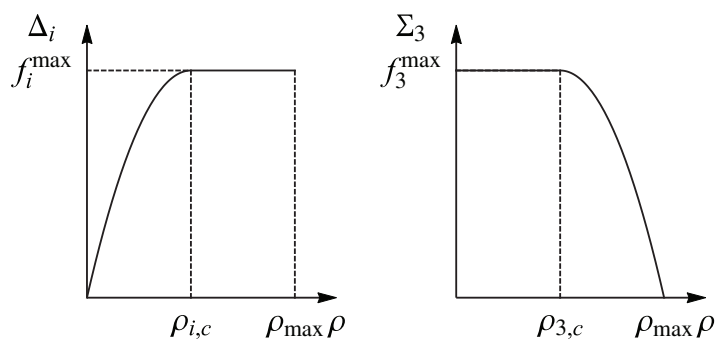

Figure 1. The equilibrium demand and supply functions.

- for $i \in \mathrm{I}, \rho_{i, 0} \in\left[0, \rho_{\max }\right]$ is a good datum if $\rho_{i, 0} \geq \rho_{i, c}$;

- $\rho_{3,0} \in\left[0, \rho_{\max }\right]$ is a good datum if $\rho_{3,0} \leq \rho_{3, c}$;

- for $h \in \mathrm{H}, \rho_{h, 0} \in\left[0, \rho_{\max }\right]$ is a bad datum if it is not a good datum.

With a slight abuse of notation, in the following we write $\rho_{h, 0} \in \mathrm{G}$ if it is a good datum, $\vec{\rho}_{0}=\left(\rho_{1,0}, \rho_{2,0}, \rho_{3,0}\right) \in$ GGG if each component of $\vec{\rho}_{0}$ is a good datum, and so on.

Definition 2.4 (Sect. 5.2.3 in [20]). For $i \in \mathrm{I}$, the equilibrium demand function (sometimes called equilibrium sending capacity) of the $i$ th incoming road $\Omega_{i}$ is the map $\Delta_{i}:\left[0, \rho_{\max }\right] \rightarrow\left[0, f_{i}^{\max }\right]$, see Figure 1 , defined by

$$
\Delta_{i}(\rho)= \begin{cases}f_{i}^{\max } & \text { if } \rho \text { is a good datum } \\ f_{i}(\rho) & \text { otherwise }\end{cases}
$$

The equilibrium supply function (sometimes called equilibrium receiving capacity) of the outgoing road $\Omega_{3}$ is the map $\Sigma_{3}:\left[0, \rho_{\max }\right] \rightarrow\left[0, f_{3}^{\max }\right]$, see Figure 1 , defined by

$$
\Sigma_{3}(\rho)= \begin{cases}f_{3}^{\max } & \text { if } \rho \text { is a good datum } \\ f_{3}(\rho) & \text { otherwise. }\end{cases}
$$

Definition 2.5 (Def. 2.5 in [8]). We say that $\vec{\rho}_{0} \in \Lambda$ is an equilibrium for a Riemann solver at the junction $\mathcal{R S}$ if $\mathcal{R S}\left[\vec{\rho}_{0}\right] \equiv \vec{\rho}_{0}$; as a consequence the constant valued function $(t, x) \mapsto \vec{\rho}_{0}$ is a stationary solution.

Definition 2.6. Let $\mathcal{R S}: \Lambda \rightarrow \mathrm{BV}(\mathcal{N} ; \Lambda)$ be a Riemann solver at the junction.

- We say that $\mathcal{R S}$ has the property (P1) (see [21], Def. 8) if $\gamma\left(\mathcal{R S}\left[\vec{\rho}_{0}\right]\right)=\gamma\left(\mathcal{R S}\left[\vec{\rho}_{0}^{*}\right]\right.$ ) for any initial data $\vec{\rho}_{0}, \vec{\rho}_{0}^{*} \in \Lambda$ such that $\rho_{h, 0}=\rho_{h, 0}^{*}$ whenever $\rho_{h, 0}$ or $\rho_{h, 0}^{*}$ is a bad datum, $h \in \mathrm{H}$.

- We say that $\mathcal{R S}$ is consistent (see [20], p. 72) if for any initial datum $\vec{\rho}_{0} \in \Lambda$ the vector of the traces $\gamma\left(\mathcal{R S}\left[\vec{\rho}_{0}\right]\right)$ is an equilibrium for $\mathcal{R S}$ in the sense of Definition 2.5.

- We say that $\mathcal{R S}$ is $\mathrm{L}_{\text {loc }}^{1}$-continuous at $\vec{\rho}_{0} \in \Lambda$ if $\mathcal{R} \mathcal{S}_{h}$ is $\mathrm{L}_{\text {loc }}^{1}$-continuous at $\rho_{h, 0}$ for all $h \in \mathrm{H}$.

In Definition 2.7 below we introduce the general form of the Riemann solvers at the junction considered in this paper, namely we introduce the concept of admissibility. We use the following notation:

- for $h \in \mathrm{H}, \mathcal{R}_{h}$ is the Lax Riemann solver [7] associated to (2.1);

- for $i \in \mathrm{I}, \hat{\rho}_{i} \in \mathrm{C}^{0}\left(\left[0, f_{i}^{\max }\right] ;\left[\rho_{i, c}, \rho_{\max }\right]\right)$ is the inverse function of $\left.f_{i}\right|_{\left[\rho_{i, c}, \rho_{\max }\right]}$;

- $\check{\rho}_{3} \in \mathrm{C}^{0}\left(\left[0, f_{3}^{\max }\right] ;\left[0, \rho_{3, c}\right]\right)$ is the inverse function of $\left.f_{3}\right|_{\left[0, \rho_{3, c}\right]}$. 


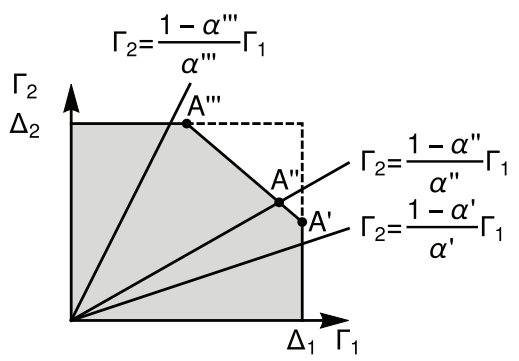

Figure 2. The geometrical meaning of (2.5) for $\alpha \in\left\{\alpha^{\prime}, \alpha^{\prime \prime}, \alpha^{\prime \prime \prime}\right\} \subset[0,1]$ such that $\alpha^{\prime \prime}, Q\left(\vec{\rho}_{0}\right)<$ $Q\left(\vec{\rho}_{0}\right)-\Delta_{2}\left(\rho_{2,0}\right)<\alpha^{\prime \prime} Q\left(\vec{\rho}_{0}\right)<\Delta_{1}\left(\rho_{1,0}\right)<\alpha^{\prime} Q\left(\vec{\rho}_{0}\right)$. The gray area corresponds to the attainable values for $\left(\Gamma_{1}, \Gamma_{2}\right)$, namely to the region $\left\{\left(\Gamma_{1}, \Gamma_{2}\right) \in\left[0, \Delta_{1}\left(\rho_{1,0}\right)\right] \times\left[0, \Delta_{2}\left(\rho_{2,0}\right)\right]: \Gamma_{1}+\Gamma_{2} \leq\right.$ $\left.Q\left(\vec{\rho}_{0}\right)\right\}$. The $\operatorname{dot} A^{\prime}$ represents the passing flow corresponding to the priority coefficient $\alpha^{\prime}$, and so on. In the figure we let $\Delta_{i}=\Delta_{i}\left(\rho_{i, 0}\right), i \in \mathrm{I}$.

Definition 2.7. For any fixed priority factor $\alpha \in[0,1]$ and receiving capacity $Q: \Lambda \rightarrow\left[0, f_{3}^{\max }\right]$ such that $Q(\vec{\rho}) \leq \Sigma_{3}\left(\rho_{3}\right)$ for any $\vec{\rho} \in \Lambda$, we say that a Riemann solver at the junction $\mathcal{R}_{j}: \Lambda \rightarrow \mathrm{BV}(\mathcal{N} ; \Lambda)$ is admissible if it has the form

$$
\vec{\rho}_{0} \mapsto\left(\mathcal{R}_{1}\left[\rho_{1,0}, \hat{\rho}_{1}\left(\Gamma_{1}\left(\vec{\rho}_{0}\right)\right)\right], \mathcal{R}_{2}\left[\rho_{2,0}, \hat{\rho}_{2}\left(\Gamma_{2}\left(\vec{\rho}_{0}\right)\right)\right], \mathcal{R}_{3}\left[\check{\rho}_{3}\left(\Gamma_{1}\left(\vec{\rho}_{0}\right)+\Gamma_{2}\left(\vec{\rho}_{0}\right)\right), \rho_{3,0}\right]\right),
$$

where $\Gamma_{i}: \Lambda \rightarrow\left[0, f_{i}^{\max }\right], i \in \mathrm{I}$, are the passing flow at the junction from the $i$ th road corresponding to the receiving capacity $Q=Q(\vec{\rho})$, and are defined by

$$
\Gamma_{1} \equiv\left\{\begin{array} { l l } 
{ \Delta _ { 1 } } & { \text { if } \Delta _ { 1 } + \Delta _ { 2 } \leq Q , } \\
{ \Delta _ { 1 } } & { \text { if } \alpha Q \geq \Delta _ { 1 } , }
\end{array} \quad \Gamma _ { 2 } \equiv \left\{\begin{array}{ll}
\Delta_{2} & \text { if } \Delta_{1}+\Delta_{2} \leq Q \\
Q-\Gamma_{1} & \text { otherwise }
\end{array}\right.\right.
$$

where $\Delta_{i}$ stands for $\Delta_{i}\left(\rho_{i, 0}\right), i \in \mathrm{I}$.

In other words, for $\vec{\rho}_{0} \in \Lambda$, we have that $\Gamma_{i}\left(\vec{\rho}_{0}\right), i \in \mathrm{I}$, are defined as follows:

- if the total sending capacity of the incoming roads $\Delta_{1}\left(\rho_{1,0}\right)+\Delta_{2}\left(\rho_{2,0}\right)$ does not exceed $Q\left(\vec{\rho}_{0}\right)$, then $\Gamma_{i}\left(\vec{\rho}_{0}\right)=$ $\Delta_{i}\left(\rho_{i, 0}\right), i \in \mathrm{I}$;

- otherwise, the passing flow at the junction $\Gamma_{1}\left(\vec{\rho}_{0}\right)+\Gamma_{2}\left(\vec{\rho}_{0}\right)$ coincides with $Q\left(\vec{\rho}_{0}\right)$ and is split between the incoming roads in accordance with the priority factor $\alpha$, see Figure 2.

We observe that $\Gamma_{1}$ defined in $(2.5)$ can be rewritten in a more compact form as follows

$$
\Gamma_{1} \equiv \begin{cases}\Delta_{1} & \text { if } \Delta_{1}+\Delta_{2} \leq Q \\ \max \left\{Q-\Delta_{2}, \min \left\{\alpha Q, \Delta_{1}\right\}\right\} & \text { if } \Delta_{1}+\Delta_{2}>Q\end{cases}
$$

Clearly, in the present setting choosing an admissible Riemann solver at the junction is equivalent to choosing a priority factor $\alpha \in[0,1]$ and a receiving capacity $Q: \Lambda \rightarrow\left[0, f_{3}^{\max }\right]$ such that $Q(\vec{\rho}) \leq \Sigma_{3}\left(\rho_{3}\right)$ for any $\vec{\rho} \in \Lambda$.

Notice that an admissible Riemann solver at the junction $\mathcal{R}_{j}$ associates to any road-wise constant initial condition $\vec{\rho}_{0} \in \Lambda$, the self-similar weak solution $\mathcal{R}_{j}\left[\vec{\rho}_{0}\right]$ in the network $\mathcal{N}$ realizing the maximum of the passing flow at the junction because

$$
\Gamma_{1}\left(\vec{\rho}_{0}\right)+\Gamma_{2}\left(\vec{\rho}_{0}\right)= \begin{cases}\Delta_{1}\left(\rho_{1,0}\right)+\Delta_{2}\left(\rho_{2,0}\right) & \text { if } \Delta_{1}\left(\rho_{1,0}\right)+\Delta_{2}\left(\rho_{2,0}\right) \leq Q\left(\vec{\rho}_{0}\right), \\ Q\left(\vec{\rho}_{0}\right), & \text { otherwise. }\end{cases}
$$


Remark 2.8. The output of an admissible Riemann solver at the junction $\mathcal{R}_{j}$ can be understood as a "collection" of solutions to three initial-boundary value problems (one for each road) coupled through their boundary conditions

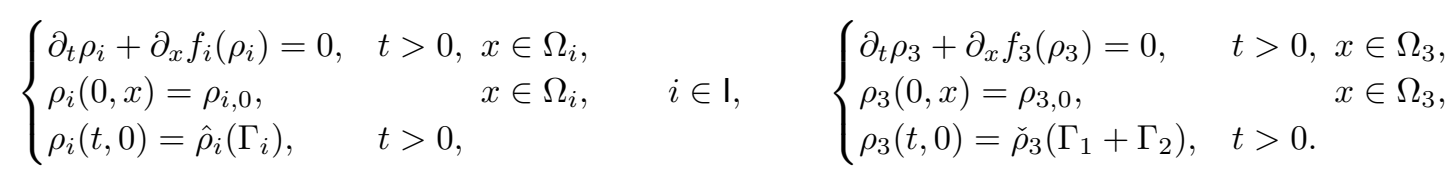

We recall that the solutions to the initial-boundary value problems (2.7) coincide with the restrictions to $\Omega_{h}$ of the Kruzhkov [23] entropy solutions, constructed via the Lax Riemann solver, to the Riemann problems

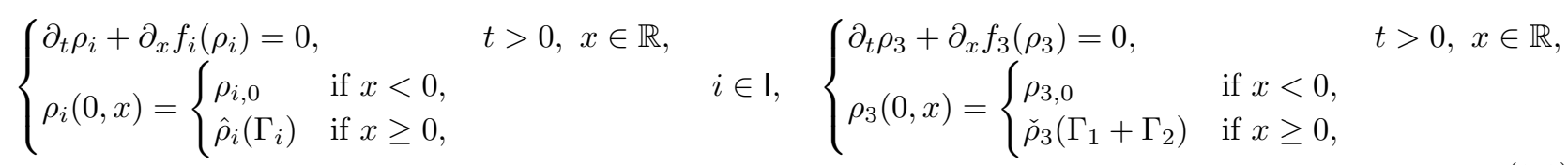

respectively. We observe that, by (2.5) and (2.6), for any $\vec{\rho}_{0} \in \Lambda$ we have

$$
\Gamma_{i}\left(\vec{\rho}_{0}\right) \leq \Delta_{i}\left(\rho_{i, 0}\right), \quad i \in \mathrm{I}, \quad \Gamma_{1}\left(\vec{\rho}_{0}\right)+\Gamma_{2}\left(\vec{\rho}_{0}\right) \leq Q\left(\vec{\rho}_{0}\right) \leq \Sigma_{3}\left(\rho_{3,0}\right) .
$$

Therefore the traces $\gamma\left(\mathcal{R}_{j}\left[\vec{\rho}_{0}\right]\right)=\left(\bar{\rho}_{1}, \bar{\rho}_{2}, \bar{\rho}_{3}\right)$ satisfy for a.e. $t>0$

$$
f_{i}\left(\bar{\rho}_{i}(t)\right)=\Gamma_{i}\left(\vec{\rho}_{0}\right), \quad i \in \mathrm{I}, \quad f_{3}\left(\bar{\rho}_{3}(t)\right)=\Gamma_{1}\left(\vec{\rho}_{0}\right)+\Gamma_{2}\left(\vec{\rho}_{0}\right),
$$

but not necessarily $\bar{\rho}_{i}(t)=\hat{\rho}_{i}\left(\Gamma_{i}\right), i \in \mathrm{I}$, or $\bar{\rho}_{3}(t)=\check{\rho}_{3}\left(\Gamma_{1}+\Gamma_{2}\right)$, see [7].

With a slight abuse of notations, we denote by $\mathcal{R}_{h}, h \in \mathrm{H}$, the Lax Riemann solvers associated to the initial-boundary value problems (2.7) or to the Riemann problems (2.8).

In Definition 2.9 below we introduce three admissible Riemann solvers at the junction. Each of them is characterized by a different receiving capacity $Q$. From now on, we assume that

$$
f_{3}^{\max }<f_{1}^{\max }+f_{2}^{\max }
$$

and we introduce the constraint function $g:\left[0, f_{1}^{\max }+f_{2}^{\max }\right] \rightarrow\left[0, f_{3}^{\max }\right]$ defined by

$$
g(s)= \begin{cases}f_{3}^{\max } & \text { if } s \leq f_{3}^{\max } \\ f_{3}^{\max }+\frac{g_{\min }-f_{3}^{\max }}{b-f_{3}^{\max }}\left(s-f_{3}^{\max }\right) & \text { if } f_{3}^{\max }<s<b \\ g_{\min } & \text { otherwise }\end{cases}
$$

where $b \in\left(f_{3}^{\max }, f_{1}^{\max }+f_{2}^{\max }\right]$ and $g_{\min } \in\left(0, f_{3}^{\max }\right)$.

Definition 2.9. Let $\alpha \in[0,1]$ be a priority factor and $g$ be a constraint function as in (2.11).

- We denote by $\mathcal{R}_{j}^{\mathrm{CGP}}: \Lambda \rightarrow \mathrm{BV}(\mathcal{N} ; \Lambda)$ the admissible Riemann solver at the junction introduced in [12] and corresponding to the receiving capacity $Q \equiv \Sigma_{3}$, where $\Sigma_{3}$ is the equilibrium receiving capacity defined by (2.3).

- We denote by $\mathcal{R}_{j}^{\mathrm{HBC}}: \Lambda \rightarrow \mathrm{BV}(\mathcal{N} ; \Lambda)$ the admissible Riemann solver at the junction introduced in [22] and corresponding to the effective receiving capacity $Q^{\mathrm{HBC}}: \Lambda \rightarrow\left[0, f_{3}^{\max }\right]$ defined by

$$
Q^{\mathrm{HBC}}\left(\vec{\rho}_{0}\right)=\min \left\{\Sigma_{3}\left(\rho_{3,0}\right), g\left(\Delta_{1}\left(\rho_{1,0}\right)+\Delta_{2}\left(\rho_{2,0}\right)\right)\right\} .
$$


- We denote by $\mathcal{R}_{j}^{l}: \Lambda \rightarrow \mathrm{BV}(\mathcal{N} ; \Lambda)$ the admissible Riemann solver at the junction corresponding to the local effective receiving capacity $Q^{l}: \Lambda \rightarrow\left[0, f_{3}^{\max }\right]$ defined by

$$
Q^{l}\left(\vec{\rho}_{0}\right)=\min \left\{Q^{\mathrm{HBC}}\left(\vec{\rho}_{0}\right), Q^{\mathrm{HBC}}\left(\mathcal{T}\left[\vec{\rho}_{0}\right]\right), Q^{\mathrm{HBC}}\left(\mathcal{T}^{2}\left[\vec{\rho}_{0}\right]\right)\right\}
$$

where $\mathcal{T}=\gamma \circ \mathcal{R}_{j}^{\mathrm{HBC}}$.

We observe that by definition $Q^{l}\left(\vec{\rho}_{0}\right) \leq Q^{\mathrm{HBC}}\left(\vec{\rho}_{0}\right) \leq \Sigma_{3}\left(\rho_{3,0}\right) \leq f_{3}^{\text {max }}$. In particular, this ensures that the Riemann solvers at the junction introduced in Definition 2.9 are admissible.

Remark 2.10. We stress that, whenever $\Delta_{1}\left(\rho_{1,0}\right)+\Delta_{2}\left(\rho_{2,0}\right)$ is smaller than $f_{3}^{\max }$, the effective receiving capacity $Q^{\mathrm{HBC}}\left(\vec{\rho}_{0}\right)$ coincides with the equilibrium receiving capacity $\Sigma_{3}\left(\rho_{3,0}\right)$ and $\mathcal{R}_{j}^{\mathrm{HBC}}\left[\vec{\rho}_{0}\right] \equiv \mathcal{R}_{j}^{\mathrm{CGP}}\left[\vec{\rho}_{0}\right]$.

The solver $\mathcal{R}_{j}^{\mathrm{CGP}}$ does not represent any capacity drop effect and for this reason Haut, Bastin and Chitour introduced $\mathcal{R}_{j}^{\mathrm{HBC}}$, see [22]. Roughly speaking, the Riemann solver at the junction $\mathcal{R}_{j}^{\mathrm{HBC}}$ accounts for the capacity drop effect by taking $Q \equiv Q^{\mathrm{HBC}}\left(\vec{\rho}_{0}\right)$ instead of $Q \equiv \Sigma_{3}\left(\rho_{3,0}\right)$ in Definition 2.7.

We list below two drawbacks of $\mathcal{R}_{j}^{\mathrm{HBC}}$.

(D.I) The main drawback (at least from the mathematical point of view) is that $\mathcal{R}_{j}^{\mathrm{HBC}}$ is not consistent, see Section 6.1 for an explicit example. Roughly speaking, let $\gamma(\vec{\rho})=\left(\bar{\rho}_{1}, \bar{\rho}_{2}, \bar{\rho}_{3}\right)$ be the vector of traces at $x=0$ of the solution $\vec{\rho}=\mathcal{R}_{j}^{\mathrm{HBC}}\left[\vec{\rho}_{0}\right]$ corresponding to an initial datum $\vec{\rho}_{0} \in \mathrm{BBG}$ such that $\Sigma_{3}\left(\rho_{3,0}\right) \leq$ $\Delta_{1}\left(\rho_{1,0}\right)+\Delta_{2}\left(\rho_{2,0}\right)<b$. By definition $Q^{\mathrm{HBC}}\left(\vec{\rho}_{0}\right)=g\left(\Delta_{1}\left(\rho_{1,0}\right)+\Delta_{2}\left(\rho_{2,0}\right)\right) \leq f_{3}^{\max }=\Sigma_{3}\left(\rho_{3,0}\right)$. It may happen that $\gamma(\vec{\rho})$ is not an equilibrium. If, for $i \in \mathrm{I}, \bar{\rho}_{i}=\hat{\rho}_{i}$, which by definition is good datum, then $g\left(\Delta_{1}\left(\bar{\rho}_{1}\right)+\Delta_{2}\left(\bar{\rho}_{2}\right)\right)<g\left(\Delta_{1}\left(\rho_{1,0}\right)+\Delta_{2}\left(\rho_{2,0}\right)\right)$ because $g$ is decreasing. Thus the constraint diminishes and $\gamma(\vec{\rho})$ does not satisfy it.

(D.II) As already observed in [22], the solution associated to $\mathcal{R}_{j}^{\mathrm{HBC}}$ may develop a traffic jam that persists forever, even if the same initial condition leads to very moderate congestion in the solution associated to $\mathcal{R}_{j}^{C G P}$ (without capacity drop representation).

We fix the drawback (D.I) by introducing $\mathcal{R}_{j}^{l}$, see Section 3.

In the setting of crowd dynamics, the model proposed in [16], featuring a point constraint depending on a point value of the density, suffers from a problem similar to (D.II). In [5] the authors showed that this issue can be overcome by considering a point constraint whose value at each time $t>0$ depends on the average value of the solution on an interval. In this paper we propose an analogous approach to obtain a more realistic representation of the transient behavior between congested and free traffic at a merge. Roughly speaking, according to the model we describe in Section 4 the effective receiving capacity of the junction depends on the average density of vehicles on the incoming roads in an upstream neighborhood of the intersection, and not merely on the traces of the density functions at $x=0$.

In the following theorem we collect the main properties of the admissible Riemann solvers at the junction introduced in Definition 2.9; the proof is deferred to Section 6.

Theorem 2.11. - The Riemann solver at the junction $\mathcal{R}_{j}^{\mathrm{CGP}}$ has the property (P1), is consistent, is $\mathrm{L}_{\text {loc }}^{1}{ }^{-}$ continuous, but does not reproduce the capacity drop at the junction.

- The Riemann solver at the junction $\mathcal{R}_{j}^{\mathrm{HBC}}$ has the property (P1), reproduces the capacity drop at the junction but is not consistent.

- The Riemann solver at the junction $\mathcal{R}_{j}^{l}$ has the property (P1), is consistent and reproduces the capacity drop at the junction but it fails to be $\mathrm{L}_{\text {loc-continuous. }}^{1}$

Remark 2.12. In the proof of Theorem 2.11 we give an explicit example to show that $\mathcal{R}_{j}^{l}$ is not $\mathrm{L}_{\text {loc }}^{1}$-continuous. We can observe that in the same situation $\mathcal{R}_{j}^{\mathrm{HBC}}$ is $\mathrm{L}_{\text {loc }}^{1}$-continuous, but we do not address the $\mathrm{L}_{\text {loc }}^{1}$-continuity of the solver $\mathcal{R}_{j}^{\mathrm{HBC}}$ in this paper. 


\section{The admissible Riemann solver at the Junction $\mathcal{R}_{j}^{l}$}

In this section we give a more explicit description of $\mathcal{R}_{j}^{l}$. As already pointed out in (D.I), we introduce $\mathcal{R}_{j}^{l}$ to overcome the non-consistency of the Riemann solver $\mathcal{R}_{j}^{\mathrm{HBC}}$. The lack of such property has consequences not only from a mathematical point of view (as it means that the solver provides non-stable solutions) but also in numerical simulations. Indeed, if we implement $\mathcal{R}_{j}^{\mathrm{HBC}}$ in a finite volumes numerical scheme we do not observe the expected solution, as it is destroyed after a few time iterations, but we observe the solution corresponding to $\mathcal{R}_{j}^{l}$, see Section 5 for the description of our scheme. Therefore, it makes sense to introduce the iterate version of $\mathcal{R}_{j}^{\mathrm{HBC}}$ and study its properties.

We recall that our analysis, which relies on a case-by-case study, heavily depends on assumptions (2.10) and (2.11). In particular, we prove that, for $\mathcal{R}_{j}^{l}$, Definition 2.9 is equivalent to say that $\mathcal{R}_{j}^{l}=\mathcal{R}_{j}^{\mathrm{HBC}} \circ \mathcal{T}^{2}$, where $\mathcal{T}$ is the composition of the Riemann solver $\mathcal{R}_{j}^{\mathrm{HBC}}$ and the trace operator $\gamma$, that is

$$
\begin{aligned}
\mathcal{T}=\gamma \circ \mathcal{R}_{j}^{\mathrm{HBC}}: & \Lambda \\
\vec{\rho}_{0} & \mapsto \gamma\left(\mathcal{R}_{j}^{\mathrm{HBC}}\left[\vec{\rho}_{0}\right]\right) .
\end{aligned}
$$

Moreover, by the same analysis we have the consistency of $\mathcal{R}_{j}^{l}$ and a more explicit definition of $\mathcal{R}_{j}^{l}$, which associates to any initial condition the corresponding solution without a direct computation of the iterations of $\mathcal{T}$, see Proposition 3.1.

If assumptions (2.10) and (2.11) are not enforced, three iterations might not be sufficient to achieve consistency, additional cases need to be discussed and, of course, Proposition 3.1 does not hold.

Whenever the following quantities make sense, we denote

$$
\begin{array}{lll}
\hat{\rho}_{1, g}=\hat{\rho}_{1}\left(g_{\min }-f_{2}^{\max }\right), & \hat{\rho}_{2, g}=\hat{\rho}_{2}\left(g_{\min }-f_{1}^{\max }\right), & \check{\rho}_{3, g}=\check{\rho}_{3}\left(g_{\min }\right), \\
\hat{\rho}_{1, \alpha}=\hat{\rho}_{1}\left(\alpha g_{\min }\right), & \hat{\rho}_{2, \alpha}=\hat{\rho}_{2}\left((1-\alpha) g_{\min }\right), & \\
\vec{\rho}_{A}=\left(\hat{\rho}_{1, g}, \rho_{2, c}, \check{\rho}_{3, g}\right), & \vec{\rho}_{B}=\left(\hat{\rho}_{1, \alpha}, \hat{\rho}_{2, \alpha}, \check{\rho}_{3, g}\right), & \vec{\rho}_{C}=\left(\rho_{1, c}, \hat{\rho}_{2, g}, \check{\rho}_{3, g}\right) .
\end{array}
$$

Notice that by (2.10) and (2.11) we have $g_{\min } \leq f_{3}^{\max }<f_{1}^{\max }+f_{2}^{\max }$, whence $g_{\min }-f_{2}^{\max }<f_{1}^{\max }$ and $g_{\min }-f_{1}^{\max }<f_{2}^{\max }$.

Proposition 3.1. The Riemann solver $\mathcal{R}_{j}^{l}: \Lambda \rightarrow \mathrm{BV}(\mathcal{N} ; \Lambda)$ behaves as follows.

(a) If $\left(\rho_{1,0}, \rho_{2,0}\right) \in \mathrm{BG}, Q^{\mathrm{HBC}}\left(\vec{\rho}_{0}\right)=g\left(f_{1}\left(\rho_{1,0}\right)+f_{2}^{\max }\right)$ and $f_{1}\left(\rho_{1,0}\right)<\alpha Q^{\mathrm{HBC}}\left(\vec{\rho}_{0}\right)$, then

$$
Q^{l}\left(\vec{\rho}_{0}\right)=Q^{\mathrm{HBC}}\left(\vec{\rho}_{0}\right)
$$

and

$$
\gamma \circ \mathcal{R}_{j}^{l}\left[\vec{\rho}_{0}\right]=\mathcal{T}\left[\vec{\rho}_{0}\right]=\left(\rho_{1,0}, \hat{\rho}_{2}\left(Q^{l}\left(\vec{\rho}_{0}\right)-f_{1}\left(\rho_{1,0}\right)\right), \check{\rho}_{3}\left(Q^{l}\left(\vec{\rho}_{0}\right)\right)\right) .
$$

(b) If $\left(\rho_{1,0}, \rho_{2,0}\right) \in \mathrm{GB}, Q^{\mathrm{HBC}}\left(\vec{\rho}_{0}\right)=g\left(f_{1}^{\max }+f_{2}\left(\rho_{2,0}\right)\right)$ and $f_{2}\left(\rho_{2,0}\right)<(1-\alpha) Q^{\mathrm{HBC}}\left(\vec{\rho}_{0}\right)$, then

$$
Q^{l}\left(\vec{\rho}_{0}\right)=Q^{\mathrm{HBC}}\left(\vec{\rho}_{0}\right)
$$

and

$$
\gamma \circ \mathcal{R}_{j}^{l}\left[\vec{\rho}_{0}\right]=\mathcal{T}\left[\vec{\rho}_{0}\right]=\left(\hat{\rho}_{1}\left(Q^{l}\left(\vec{\rho}_{0}\right)-f_{2}\left(\rho_{2,0}\right)\right), \rho_{2,0}, \check{\rho}_{3}\left(Q^{l}\left(\vec{\rho}_{0}\right)\right)\right) .
$$

(c) If $\left(\rho_{1,0}, \rho_{2,0}\right) \in \mathrm{BB}, Q^{\mathrm{HBC}}\left(\vec{\rho}_{0}\right)>g\left(f_{1}\left(\rho_{1,0}\right)+f_{2}^{\max }\right)$ and $f_{1}\left(\rho_{1,0}\right)<\alpha g\left(f_{1}\left(\rho_{1,0}\right)+f_{2}^{\max }\right)$, then

$$
Q^{l}\left(\vec{\rho}_{0}\right)=Q^{\mathrm{HBC}}\left(\mathcal{T}\left[\vec{\rho}_{0}\right]\right)=g\left(f_{1}\left(\rho_{1,0}\right)+f_{2}^{\max }\right),
$$

and

$$
\gamma \circ \mathcal{R}_{j}^{l}\left[\vec{\rho}_{0}\right]=\mathcal{T}\left[\vec{\rho}_{0}\right]=\left(\rho_{1,0}, \hat{\rho}_{2}\left(Q^{l}\left(\vec{\rho}_{0}\right)-f_{1}\left(\rho_{1,0}\right)\right), \check{\rho}_{3}\left(Q^{l}\left(\vec{\rho}_{0}\right)\right)\right)
$$


TABle 1. The correspondence between the cases of Proposition 3.1 and those of Proposition 6.2.

\begin{tabular}{ll}
\hline \hline Proposition 3.1 & Proposition 6.2 \\
\hline Case (a) & (BGG.i-1) (BGB.iii-1) \\
Case (b) & (GBG.i-3) (GBB.iii-3) \\
Case (c) & (BBG.ii-1) (BBB.iii-1) (BBB.iv-1) \\
Case (d) & (BBG.ii-4.a) (BBB.ii-4.a) (BBB.iv-6.a) \\
Case (e) & (GGB.i) (BGB.iv-1) (GBG.iv-2) (BGB.iv-4) (BBB.iv-3) (BBB.iv-4) (BGG.ii) \\
& (BBG.i) (BGB.i) (BGB.ii) (BBB.ii) (BBB.i) (GBG.ii) (GBB.i) (GBB.ii) \\
& (GBB.iv-1) (GBB.iv-2) (GBB.iv-4) (BBB.iv-6.b) \\
Case (f) & (GGG) (BGG.i-2) (BGG.i-3) (BGG.i-4) (GGB.ii) (BBG.ii-2) (BBG.ii-3) \\
& (BGB.iii-2) (BGB.iii-3) (BGB.iv-3) (BGB.iv-5) (BBB.iii-2) (BBB.iii-3) \\
& (BBB.iv-2) (BBB.iv-5) (BBG.ii-4.b) (BBB.iii-4.b) (BBB.iv-6.c) (GBG.i-2) \\
& (GBG.i-1) (GBG.i-4) (GBB.iii-1) (GBB.iii-2) (GBB.iv-3) (GBB.iv-5) \\
\hline
\end{tabular}

(d) If $\left(\rho_{1,0}, \rho_{2,0}\right) \in \mathrm{BB}, Q^{\mathrm{HBC}}\left(\vec{\rho}_{0}\right)>g\left(f_{1}^{\max }+f_{2}\left(\rho_{2,0}\right)\right)$ and $f_{2}\left(\rho_{2,0}\right)<(1-\alpha) g\left(f_{1}^{\max }+f_{2}\left(\rho_{2,0}\right)\right)$, then

$$
Q^{l}\left(\vec{\rho}_{0}\right)=Q^{\mathrm{HBC}}\left(\mathcal{T}\left[\vec{\rho}_{0}\right]\right)=g\left(f_{1}^{\max }+f_{2}\left(\rho_{2,0}\right)\right),
$$

and

$$
\gamma \circ \mathcal{R}_{j}^{l}\left[\vec{\rho}_{0}\right]=\mathcal{T}\left[\vec{\rho}_{0}\right]=\left(\hat{\rho}_{1}\left(Q^{l}\left(\vec{\rho}_{0}\right)-f_{2}\left(\rho_{2,0}\right)\right), \rho_{2,0}, \check{\rho}_{3}\left(Q^{l}\left(\vec{\rho}_{0}\right)\right)\right) .
$$

(e) If $\Sigma_{3}\left(\rho_{3,0}\right) \leq \min \left\{\Delta_{1}\left(\rho_{1,0}\right)+\Delta_{2}\left(\rho_{2,0}\right), g\left(\Delta_{1}\left(\rho_{1,0}\right)+\Delta_{2}\left(\rho_{2,0}\right)\right)\right\}$, then

$$
Q^{l}\left(\vec{\rho}_{0}\right)=Q^{\mathrm{HBC}}\left(\vec{\rho}_{0}\right)=\Sigma_{3}\left(\rho_{3,0}\right),
$$

and $\mathcal{R}_{j}^{l}\left[\vec{\rho}_{0}\right] \equiv \mathcal{R}_{j}^{\mathrm{HBC}}\left[\vec{\rho}_{0}\right] \equiv \mathcal{R}_{j}^{\mathrm{CGP}}\left[\vec{\rho}_{0}\right]$.

(f) In all other cases $Q^{l}\left(\vec{\rho}_{0}\right)=g_{\min }$ and

$$
\gamma \circ \mathcal{R}_{j}^{l}\left[\vec{\rho}_{0}\right]=\mathcal{T}\left[\vec{\rho}_{0}\right]= \begin{cases}\vec{\rho}_{A} & \text { if } \alpha g_{\min } \in\left[0, g_{\min }-f_{2}^{\max }\right], \\ \vec{\rho}_{B} & \text { if } \alpha g_{\min } \in\left(g_{\min }-f_{2}^{\max }, f_{1}^{\max }\right), \\ \vec{\rho}_{C} & \text { if } \alpha g_{\min } \in\left[f_{1}^{\max }, f_{3}^{\max }\right] .\end{cases}
$$

The proof consists of the case study deferred to Section 6.3. For the reader's convenience we summarize in Table 1 the correspondence between the case studies and the points listed in Proposition 3.1.

\subsection{An explicit admissible solution for the local model}

This section is devoted to the computation of an explicit solution by means of the local solver $\mathcal{R}_{j}^{l}$. We use such solution in Section 5.2.1 to perform a convergence analysis of our finite volumes numerical scheme subject to a local point constraint.

We consider $f(\rho) \equiv f_{h}(\rho)=\rho(1-\rho)$ as the flux for each road. As initial condition, we choose $\rho_{1,0}(x)=$ $\chi_{[-1 / 2,0]}(x), \rho_{2,0}(x)=3 / 4 \chi_{[-1 / 4,0]}(x)$ and $\rho_{3,0}(x)=0$. We fix the priority factor $\alpha=1 / 2$ and the constraint function

$$
g(s)= \begin{cases}1 / 4 & \text { if } s \leq 1 / 4 \\ \frac{3-4 s}{8} & \text { if } 1 / 4 \leq s \leq 1 / 2\end{cases}
$$

The exact solution in Figure 3 is obtained by an explicit analysis of the wave-front interactions, with computer assisted computation of front slopes and interaction times. Everywhere in the following we denote by $\sigma\left(u_{L}, u_{R}\right)$ the speed of a shock connecting the left state $u_{L}$ to the right state $u_{R}$, computed according to the RankineHugoniot condition. 


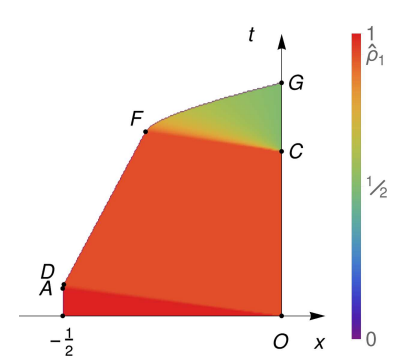

(A) The solution on $\Omega_{1}$

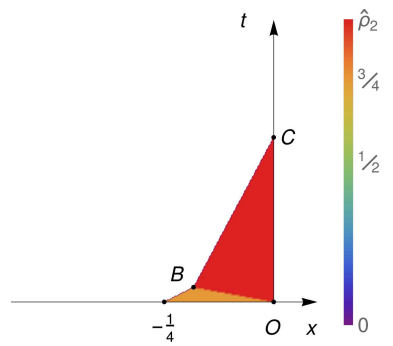

(B) The solution on $\Omega_{2}$

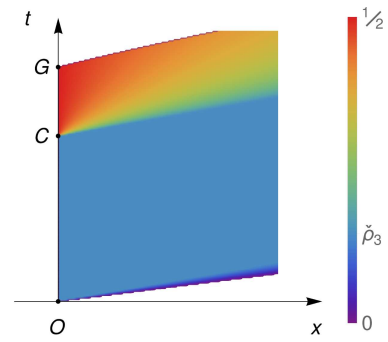

(C) The solution on $\Omega_{3}$

Figure 3. The solution in the $(x, t)$-plane obtained in Section 3.1.

At $t=0$, the local effective receiving capacity $Q^{l}$ is equal to $g_{\min }=g(1 / 2)=1 / 8$. Therefore, on $\Omega_{1}$ a rarefaction $\mathfrak{R}_{O, 1}$ starts from $O(0,0)$ and its values are given by

$$
\mathfrak{R}_{O, 1}(t, x)=\frac{1}{2}\left(1-\frac{x}{t}\right), \quad \text { for } \quad-t \leq x<-\frac{\sqrt{3}}{2} t .
$$

On $\Omega_{2}$ starts the backward shock $\mathfrak{S}_{O, 2}$ given by

$$
\mathfrak{S}_{O, 2}: \dot{x}(t)=\sigma\left(\frac{1}{2}\left(1+\frac{\sqrt{3}}{2}\right), 3 / 4\right), \quad x(0)=0 .
$$

On $\Omega_{3}$ a rarefaction starts from $O(0,0)$ and its values are given by

$$
\mathfrak{R}_{O, 3}(t, x)=\frac{1}{2}\left(1-\frac{x}{t}\right), \quad \text { for } \quad \frac{\sqrt{2}}{2} t<x \leq t .
$$

On $\Omega_{2}$, let $B\left(x_{B}, t_{B}\right)$ be the point where the shock $x(t)=-\frac{1}{4}+\frac{t}{4}$ originated from $(-1 / 4,0)$ interacts with the shock $\mathfrak{S}_{O, 2}$. As a result, from $B$ starts a shock given by

$$
\mathfrak{S}_{B, 2}: \dot{x}(t)=\sigma\left(0, \frac{1}{2}\left(1+\frac{\sqrt{3}}{2}\right)\right), \quad x\left(t_{B}\right)=x_{B},
$$

which reaches the junction in $x=0$ at time $t=t_{C}=3$ that corresponds to the time at which the second incoming road becomes empty. On $\Omega_{1}$, in $A(-1 / 2,1 / 2)$, the stationary shock originated from $(-1 / 2,0)$ interacts with the rarefaction $\mathfrak{R}_{O, 1}$. As a result, from $A$ starts a shock $\mathfrak{S}_{A, 1}$ given by

$$
\mathfrak{S}_{A, 1}: \dot{x}(t)=\sigma\left(0, \mathfrak{R}_{O, 1}(t, x(t))\right), \quad x(1 / 2)=-1 / 2 .
$$

Let $D\left(x_{D}, t_{D}\right)$ be the intersection between $\mathfrak{S}_{A, 1}$ and $x(t)=-(\sqrt{3} / 2) t$. From this point starts a forward shock

$$
\mathfrak{S}_{D, 1}: \dot{x}(t)=\sigma\left(0, \frac{1}{2}\left(1+\frac{\sqrt{3}}{2}\right)\right), \quad x\left(t_{D}\right)=x_{D} .
$$
by

At $t=t_{C}$ the local effective receiving capacity $Q^{l}$ is $g(1 / 4)=1 / 4$ and a rarefaction appears on $\Omega_{1}$. It is given

$$
\mathfrak{R}_{C, 1}(t, x)=\frac{1}{2}\left(1-\frac{x}{t-t_{C}}\right), \quad \text { for } \quad-\frac{\sqrt{3}}{2}\left(t-t_{C}\right)<x \leq 0 .
$$


Moreover, on $\Omega_{3}$ at the same time starts a rarefaction $\mathfrak{R}_{C, 3}$ given by

$$
\mathfrak{R}_{C, 3}(t, x)=\frac{1}{2}\left(1-\frac{x}{t-t_{C}}\right), \quad \text { for } \quad 0 \leq x<\frac{\sqrt{2}}{2}\left(t-t_{C}\right) .
$$

Let $F$ be the point where $\mathfrak{S}_{D, 1}$ and $\mathfrak{R}_{C, 1}$ meet together. From this point starts a forward shock $\mathfrak{S}_{F, 1}$, with left state $\rho=0$, which reaches the junction at evacuation time $t_{G} \approx 4.25$, then $\Omega_{1}$ is empty. Finally, on $\Omega_{3}$ at time $t_{G}$ starts a shock that interacts with the rarefaction $\mathfrak{R}_{C, 3}$ generating the shock

$$
\mathfrak{S}_{G, 3}: \dot{x}(t)=\sigma\left(0, \mathfrak{R}_{C, 3}(t, x(t))\right), \quad x\left(t_{G}\right)=0 .
$$

\section{A junCtion MOdel With NON-LOCAL EFFECTIVE RECEIVING CAPACity.}

The main difference between the model in this section and the ones presented above lies in the algorithm used to compute the effective receiving capacity. For any given $\vec{\rho}=\left(\rho_{1}, \rho_{2}, \rho_{3}\right)$, with $\rho_{h} \in \mathrm{L}_{\text {loc }}^{1}\left(\Omega_{h} ;\left[0, \rho_{\max }\right]\right)$ we define the non-local effective receiving capacity $Q^{\mathrm{nl}}=Q^{\mathrm{nl}}(\vec{\rho})$ by

$$
Q^{\mathrm{nl}}(\vec{\rho})=\min \left\{\Sigma_{3}\left(\bar{\rho}_{3}\right), g\left(\Delta_{1}\left(\zeta_{1}\right)+\Delta_{2}\left(\zeta_{2}\right)\right)\right\}
$$

where $\zeta_{i}$ is a weighted average of the density of vehicles on $\Omega_{i}$ in a neighborhood of the junction, namely

$$
\zeta_{i}=\int_{-\infty}^{0} w_{i}(x) \rho_{i}(x) \mathrm{d} x
$$

where $w_{i} \in \mathrm{L}^{\infty}\left(\mathbb{R}_{-} ; \mathbb{R}_{+}\right)$is an increasing function with compact support in $\left[-\ell_{i}, 0\right]$ and $\left\|w_{i}\right\|_{\mathrm{L}^{1}\left(\mathbb{R}_{-}\right)}=1, i \in \mathrm{I}$.

The concept of admissible solution introduced in the previous sections extends naturally in the following form.

Definition 4.1. Let $\alpha \in[0,1]$ be a priority factor and $\vec{\rho}=\left(\rho_{1}, \rho_{2}, \rho_{3}\right)$, with $\rho_{h} \in \mathrm{C}^{0}\left((0, \infty) ; \mathrm{L}^{1}\left(\Omega_{h} ;\left[0, \rho_{\max }\right]\right)\right) \cap$ $\operatorname{BV}_{\text {loc }}\left((0, \infty) \times \Omega_{h} ;\left[0, \rho_{\text {max }}\right]\right), h \in \mathrm{H}$, be a weak solution to (2.1) and (2.2) in the sense of Definition 2.1. We say that $\vec{\rho}$ is an admissible solution of the non-local model if the following conditions, involving the vector of traces $\gamma(\vec{\rho})=\left(\bar{\rho}_{1}, \bar{\rho}_{2}, \bar{\rho}_{3}\right)$, hold for a.e. $t$ :

$$
\begin{aligned}
& f_{1}\left(\bar{\rho}_{1}(t)\right)= \begin{cases}\Delta_{1}\left(\bar{\rho}_{1}(t)\right) & \text { if } \Delta_{1}\left(\bar{\rho}_{1}(t)\right)+\Delta_{2}\left(\bar{\rho}_{2}(t)\right) \leq \mathrm{Q}^{\mathrm{nl}}, \\
\Delta_{1}\left(\bar{\rho}_{1}(t)\right) & \text { if } \alpha \mathrm{Q}^{\mathrm{nl}} \geq \Delta_{1}\left(\bar{\rho}_{1}(t)\right), \\
\alpha Q^{\mathrm{nl}} & \text { if } \mathrm{Q}^{\mathrm{nl}}-\Delta_{2}\left(\bar{\rho}_{2}(t)\right)<\alpha \mathrm{Q}^{\mathrm{nl}}<\Delta_{1}\left(\bar{\rho}_{1}(t)\right), \quad \text { otherwise }, \\
Q^{\mathrm{nl}}-\Delta_{2}\left(\bar{\rho}_{2}(t)\right) & \text { if } \alpha \mathrm{Q}^{\mathrm{nl}} \leq \mathrm{Q}^{\mathrm{nl}}-\Delta_{2}\left(\bar{\rho}_{2}(t)\right),\end{cases} \\
& f_{2}\left(\bar{\rho}_{2}(t)\right)= \begin{cases}\Delta_{2}\left(\bar{\rho}_{2}(t)\right) & \text { if } \Delta_{1}\left(\bar{\rho}_{1}(t)\right)+\Delta_{2}\left(\bar{\rho}_{2}(t)\right) \leq \mathrm{Q}^{\mathrm{nl}}, \\
Q^{\mathrm{nl}}-f_{1}\left(\bar{\rho}_{1}(t)\right), & \text { otherwise }\end{cases}
\end{aligned}
$$

where $Q^{\mathrm{nl}}=Q^{\mathrm{nl}}(\vec{\rho}(t))$ is the non-local effective receiving capacity, computed on the profile of the solution at time $t$ and defined by (4.1).

The analytical proof of existence and stability of such admissible solutions for a general Cauchy problem is a difficult open question. In this paper we limit our attention to special situations in which the initial condition is road-wise constant or the constraint function $g$ is piecewise constant.

Conjecture 4.2. Given a constraint function $g$ as in (2.11) and fluxes $f_{h}, h \in \mathbf{H}$, satisfying (F), we can associate a unique admissible solution in $\mathrm{C}^{0}\left((0, \infty) ; \Pi_{h=1}^{3} \mathrm{~L}^{1}\left(\Omega_{h} ;\left[0, \rho_{\max }\right]\right)\right) \cap \Pi_{h=1}^{3} \mathrm{BV}_{\text {loc }}\left((0, \infty) \times \Omega_{h} ;\left[0, \rho_{\max }\right]\right)$ to any road-wise constant initial condition in $\Lambda$. We denote by $\mathcal{S}_{j}^{\text {nl }}: \Lambda \rightarrow \mathrm{C}^{0}\left([0, T] ; \Pi_{h=1}^{3} \mathrm{~L}^{1}\left(\Omega_{h} ;\left[0, \rho_{\max }\right]\right)\right) \cap$ $\Pi_{h=1}^{3} \mathrm{BV}_{\text {loc }}\left((0, \infty) \times \Omega_{h} ;\left[0, \rho_{\max }\right]\right)$ the solver operator. 
The proof of this conjecture will appear in a separate paper together with an existence result for the Cauchy problem at a merge subject to piecewise constant time dependent point constraint at the junction.

The operator $\mathcal{S}_{j}^{\mathrm{nl}}$ is not a Riemann solver as in general it does not produce self-similar solutions. In fact the effective receiving capacity might change even if no new wave hits the intersection, just because the value of the average functions $\zeta_{i}$ is not constant in time. In the next example we see that, if the initial conditions are road-wise constant and the constraint function $g$ is continuous decreasing as in (2.11), then the effective receiving capacity is a continuous function of time.

Example 4.3. Consider an initial datum $\vec{\rho}_{0} \in$ BBG such that $Q^{\mathrm{nl}}\left(\vec{\rho}_{0}\right)=g\left(\Delta_{1}\left(\zeta_{1}(0)\right)+\Delta_{2}\left(\zeta_{2}(0)\right)\right)=g\left(f_{1}\left(\rho_{1,0}\right)+\right.$ $\left.f_{2}\left(\rho_{2,0}\right)\right)$. If $\bar{\rho}_{i}=\hat{\rho}_{i}$ for at least one index $i \in \mathrm{I}$, the resulting waves are shocks with negative speed $\sigma_{i}$. In particular this means that $\hat{\rho}_{i}>\rho_{0, i}$ and the average $\zeta_{i}(t)$ is strictly increasing in time.

As the speed of propagation of any wave in the solution is finite, there exists $\delta>0$ such that if $t<\delta$, then $\zeta_{i}(t)$ are still bad data (therefore $\left.\Delta_{i}\left(\zeta_{i}(t)\right)=f_{i}\left(\zeta_{i}(t)\right)\right)$ and we have

$$
\begin{aligned}
\left|\zeta_{i}(t)-\zeta_{i}(0)\right| & =\int_{-\ell_{i}}^{0} w_{i}(x)\left(\hat{\rho}_{i} \chi_{\left[\sigma_{i} t, 0\right]}(x)+\rho_{i, 0} \chi_{\left(-\infty, \sigma_{i} t\right]}(x)-\rho_{i, 0}\right) \mathrm{d} x \\
& =\int_{-\ell_{i}}^{0} w_{i}(x)\left(\hat{\rho}_{i}-\rho_{i, 0}\right) \chi_{\left[\sigma_{i} t, 0\right]}(x) \mathrm{d} x \leq\left|f_{i}\left(\rho_{i, 0}\right)-f_{i}\left(\hat{\rho}_{i}\right)\right|\|w\|_{L^{\infty}\left(\mathbb{R}_{-}\right)} t .
\end{aligned}
$$

Hence, for any fixed $\varepsilon>0$ we get $\left|g\left(f_{1}\left(\zeta_{1}(t)\right)+f_{2}\left(\zeta_{2}(t)\right)\right)-g\left(f_{1}\left(\zeta_{1}(0)\right)+f_{2}\left(\zeta_{2}(0)\right)\right)\right|<\varepsilon$ as soon as $t \leq$ $\inf \left\{\delta, \varepsilon /\left(f_{i}^{\max }\|w\|_{L^{\infty}\left(\mathbb{R}_{-}\right)}\right)\right\}$.

Remark 4.4. Property (P1) basically states that equilibria are determined by bad data, as substituting a good initial datum with a different good datum does not change the trace of solution. For $\mathcal{S}_{j}^{\text {nl }}$ we can state an analogous property

For $t>0$ large enough $\mathcal{S}_{j}^{\mathrm{nl}}\left[\vec{\rho}_{0}\right](t, 0)=\mathcal{S}_{j}^{\mathrm{nl}}\left[\vec{\rho}_{0}^{*}\right](t, 0)$ for any initial data $\vec{\rho}_{0}, \vec{\rho}_{0}^{*} \in \Lambda$ such that $\rho_{h, 0}=\rho_{h, 0}^{*}$ whenever $\rho_{h, 0}$ or $\rho_{h, 0}^{*}$ is a bad datum, $h \in \mathrm{H}$.

This property holds because the sending capacity of an incoming road which has an initial condition in G will stay constant forever (with value $f_{i}^{\max }$ ), no matter what happens on the other roads. This means that the effective receiving capacity will only depend on the sending and receiving capacities of the other roads.

We observe that the solution produced by $\mathcal{S}_{j}^{\text {nl }}$ on the incoming roads can only contain waves with negative speed. On the incoming roads, any wave of negative speed which has a good datum on the left needs to have on the right another good datum. The average of two good data is in their convex hull, so it is again a good datum. Therefore if $\rho_{0, i} \in \mathrm{G}$ then $\zeta_{i}(t)$ will also be in $\mathrm{G}$, for all $t \geq 0$.

The fact that two different initial conditions for the outgoing road in $\mathrm{G}$ lead to the same asymptotic solution is straightforward.

If the function $g$ is piecewise constant, then the solution will be self-similar for a (possibly short) time span. In this special case $\mathcal{S}_{j}^{\text {nl }}$ can be seen as a Riemann solver locally in time, see [2]. The case in which $g$ is piecewise constant is important in view of future investigation of the Cauchy problem for this model (a combination of the operator splitting method and the wave-front tracking algorithm leads to the construction of global solutions), and it is the only case in which a solution can be computed explicitely, as we do in the next section. The explicit solution we obtain is used to validate a finite volumes numerical scheme and investigate its numerical convergence in Section 5 .

\subsection{An explicit admissible solution for the non-local model}

In this section we compute an explicit solution by means of $\mathcal{S}_{j}^{\text {nl }}$ in order to point out its properties. 


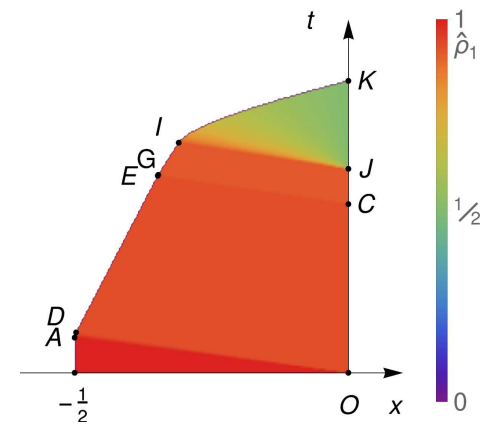

(A) The solution on $\Omega_{1}$.

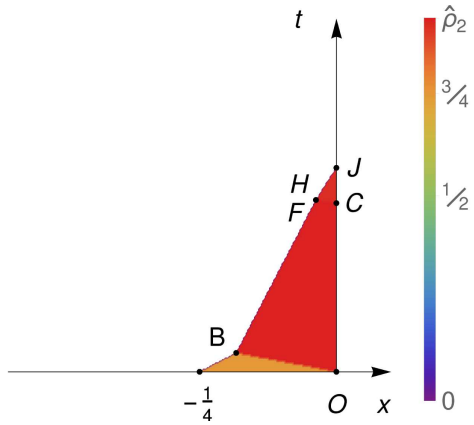

(B) The solution on $\Omega_{2}$.

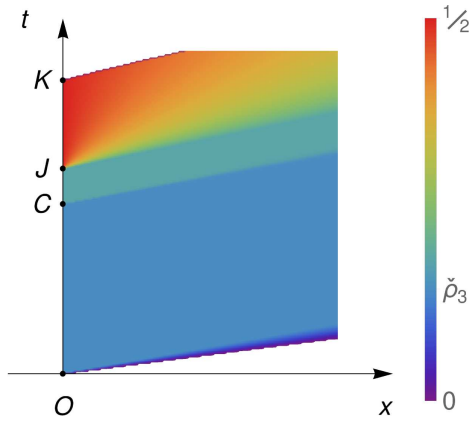

(C) The solution on $\Omega_{3}$

Figure 4. The explicit solution in the $(x, t)$-plane obtained in Section 4.1.

We consider $f(\rho) \equiv f_{h}(\rho)=\rho(1-\rho)$ as the flux for each road. As initial condition, we choose $\rho_{1,0}(x)=$ $\chi_{[-1 / 2,0]}(x), \rho_{2,0}(x)=3 / 4 \chi_{[-1 / 4,0]}(x)$ and $\rho_{3,0}(x)=0$. We fix the priority factor $\alpha=1 / 2$, the constraint function

$$
g(s)= \begin{cases}1 / 4 & \text { if } s \leq \frac{1}{4} \\ \frac{3}{20} & \text { if } \frac{1}{4}<s<\frac{9}{20} \\ \frac{1}{8} & \text { if } \frac{9}{20} \leq s \leq \frac{1}{2}\end{cases}
$$

and the weight function $w(x)=8(4 x+1) \chi_{[-1 / 4,0]}(x)$.

The exact solution is obtained by an explicit analysis of the wave-front interactions, with computer assisted computation of front slopes and interaction times presented in Figure 4.

Notice that for time smaller than the time in which the non-local effective receiving capacity $Q^{\text {nl }}$ becomes $3 / 20$, the explicit solution coincides with the solution computed in Section 3.1, therefore we omit it. With a slight abuse of notation we denote by $t=t_{C} \approx 2.40$ such time, which is obtained by solving the equation

$$
\Delta_{1}\left(\zeta_{1}(t)\right)+\Delta_{2}\left(\zeta_{2}(t)\right)=\frac{9}{20}, \quad t>t_{D}=\frac{8}{(\sqrt{3}+2)^{2}} .
$$

At this time a further rarefaction appears in each of the incoming roads. Its values are given by

$$
\mathfrak{R}_{C, i}(t, x)=\frac{1}{2}\left(1-\frac{x}{t-t_{C}}\right), \quad \text { for } \quad-\frac{\sqrt{3}}{2}\left(t-t_{C}\right)<x \leq-\frac{\sqrt{70}}{10}\left(t-t_{C}\right), \quad i \in \mathrm{I} .
$$

Moreover, on $\Omega_{3}$ at the same time starts a rarefaction $\Re_{C, 3}$ given by

$$
\mathfrak{R}_{C, 3}(t, x)=\frac{1}{2}\left(1-\frac{x}{t-t_{C}}\right), \quad \text { for } \quad \frac{\sqrt{10}}{5}\left(t-t_{C}\right)<x \leq \frac{\sqrt{2}}{2}\left(t-t_{C}\right) .
$$

On $\Omega_{1}$, let $E$ be the point where $\mathfrak{S}_{D, 1}$ and $\mathfrak{R}_{C, 1}$ meet together. From this point starts a forward shock $\mathfrak{S}_{E, 1}$, which interacts with the line $x(t)=-(\sqrt{70} / 10)\left(t-t_{C}\right)$ in $G\left(x_{G}, t_{G}\right)$ generating a forward shock

$$
\mathfrak{S}_{G, 1}: \dot{x}(t)=\sigma\left(0, \frac{1}{2}\left(1+\frac{\sqrt{70}}{10}\right)\right), \quad x\left(t_{G}\right)=x_{G} .
$$

On $\Omega_{2}$, let $F$ be the point where $\mathfrak{R}_{C, 2}$ and $\mathfrak{S}_{B, 2}$ meet. From this point starts a forward shock $\mathfrak{S}_{F, 2}$, which interacts with the line $x(t)=-(\sqrt{70} / 10)\left(t-t_{C}\right)$ in $H\left(x_{H}, t_{H}\right)$ generating a forward shock

$$
\mathfrak{S}_{H, 2}: \dot{x}(t)=\sigma\left(0, \frac{1}{2}\left(1+\frac{\sqrt{70}}{10}\right)\right), \quad x\left(t_{H}\right)=x_{H},
$$


which reaches the junction at time $t_{J} \approx 2.90$, then $\Omega_{2}$ is empty. At time $t=t_{J}$ the non-local effective receiving capacity $Q^{\text {nl }}$ attains the value $g(1 / 4)=1 / 4$ and a further rarefaction appears on $\Omega_{1}$. Its values are given by

$$
\mathfrak{R}_{J, 1}(t, x)=\frac{1}{2}\left(1-\frac{x}{t-t_{J}}\right), \quad \text { for } \quad-\frac{\sqrt{70}}{10}\left(t-t_{J}\right)<x \leq 0 .
$$

Such rarefaction interacts with $\mathfrak{S}_{G, 1}$ at $I\left(x_{I}, t_{I}\right)$ generating another shock

$$
\mathfrak{S}_{I, 1}: \dot{x}(t)=\sigma\left(0, \mathfrak{R}_{J, 1}(t, x(t))\right), \quad x\left(t_{I}\right)=x_{I},
$$

which reaches the junction at time $t=t_{K}$.

On $\Omega_{3}$, at time $t=t_{J}$ starts the rarefaction $\mathfrak{R}_{J, 3}$ given by

$$
\mathfrak{R}_{J, 3}(t, x)=\frac{1}{2}\left(1-\frac{x}{t-t_{J}}\right), \quad \text { for } \quad 0<x \leq \frac{\sqrt{10}}{5}\left(t-t_{J}\right),
$$

and finally at time $t=t_{K} \approx 4.15$ a further shock starts and interacts with $\mathfrak{R}_{J, 3}$ generating the shock

$$
\mathfrak{S}_{K, 3}: \dot{x}(t)=\sigma\left(\Re_{J, 3}(t, x(t)), 0\right), \quad x\left(t_{K}\right)=0 .
$$

\section{Finite VOlumes NUMERICAL SCHEME FOR THE CONSTRAined PROBLEM}

In this section we describe a finite volumes numerical scheme, which can be used to construct solutions for the Cauchy problem at a junction with capacity drop representation. Our scheme is developed starting from the scheme introduced in [1].

In [26] it is shown that the scheme captures the correct solution on a merge where the flux through the junction is not constrained. Then, we show that our implementation of local and non-local point constraints is correct by comparison with the explicit solution computed in Sections 3.1 and 4.1.

After that, we turn our attention to the comparison between $\mathcal{R}_{j}^{\mathrm{HBC}}, \mathcal{R}_{j}^{l}$ and $\mathcal{S}_{j}^{\mathrm{nl}}$. We reproduce the numerical simulation made in [22], then we run a simulation starting with the same initial conditions but using the nonlocal constraint at the junction. In this part we can notice that the capacity drop representation based on non-local point constraint allows to capture a more realistic behavior as the congestion disappears in finite time. Additionally, for a given constraint function $g$, we discuss the relation between the qualitative behavior of the numerical solution and the choice of the weight function $w$.

\subsection{Numerical scheme with constraint at the junction}

We fix a constant space step $\Delta x$. For $\ell \in \mathbb{Z}$ and $h \in \mathrm{H}$, we set $x_{\ell}^{h}=\ell \Delta x$. We define the cell centers $x_{\ell+\frac{1}{2}}^{h}=\left(\ell+\frac{1}{2}\right) \Delta x$ for $\ell \in \mathbb{Z}$ and consider the uniform spatial mesh on each $\Omega_{h}$

$$
\bigcup_{\ell \leq-1}\left[x_{\ell}^{i}, x_{\ell+1}^{i}\right), \quad i \in \mathrm{I}, \quad \bigcup_{\ell \geq 0}\left[x_{\ell}^{3}, x_{\ell+1}^{3}\right),
$$

so that the position of the junction $x=0$ corresponds to $x_{0}^{h}$ for each road. Then we fix a constant time step $\Delta t$ satisfying the CFL condition

$$
\Delta t \max _{h \in \mathrm{H}} L_{h} \leq \frac{\Delta x}{2},
$$

where $L_{h}$ is the Lipschitz constant of $f_{h}$. For $s \in \mathbb{N}$ we define the time discretization $t^{s}=s \Delta t$. At each time $t^{s}$, $\rho_{\ell+\frac{1}{2}}^{h, s}$ represents an approximation of the mean value of the solution on the interval $\left[x_{\ell}^{h}, x_{\ell+1}^{h}\right), \ell \in \mathbb{Z}$, along the $h$ th road. 
We initialize the scheme by discretizing the initial conditions

$$
\rho_{\ell+\frac{1}{2}}^{h, 0}=\frac{1}{\Delta x} \int_{x_{\ell}^{h}}^{x_{\ell+1}^{h}} \rho_{h, 0}(x) \mathrm{d} x,
$$

for all $h \in \mathrm{H}$ and for $\ell \leq-1$ if $h \in \mathrm{I}, \ell \geq 0$ if $h=3$.

For each $s \in \mathbb{N}$, at all cell interfaces $x_{\ell}^{h}$ with $\ell \neq 0$, we consider a monotone, consistent numerical flux $F_{h}\left(\rho_{\ell-1 / 2}^{h, s}, \rho_{\ell+1 / 2}^{h, s}\right)$ corresponding to the flux $f_{h}$. At the junction $x_{0}^{h}$ we take on each road $\Omega_{h}$ the Godunov flux $G_{h}$ corresponding to the solution of the Riemann problem at the junction computed by the appropriate solver.

Then, the finite volumes scheme can be computed by a two-step procedure:

(i) find

$$
\left(\hat{\rho}_{1}, \hat{\rho}_{2}, \check{\rho}_{3}\right) \text { such that } f_{i}\left(\hat{\rho}_{i}\right)=\Gamma_{i} \quad \text { for } i \in \mathrm{I}, \quad \text { and } \quad f_{3}\left(\check{\rho}_{3}\right)=\Gamma_{1}+\Gamma_{2},
$$

where $\Gamma_{1}$ and $\Gamma_{2}$ are defined in (2.5);

(ii) compute

$$
\rho_{\ell+\frac{1}{2}}^{h, s+1}=\rho_{\ell+\frac{1}{2}}^{h, s}-\frac{\Delta t}{\Delta x}\left(\mathcal{F}_{\ell+1}^{h, s}-\mathcal{F}_{\ell}^{h, s}\right)
$$

where

$$
\mathcal{F}_{\ell}^{h, s}= \begin{cases}F_{h}\left(\rho_{\ell-1 / 2}^{h, s}, \rho_{\ell+1 / 2}^{h, s}\right) & \text { if } h \in \mathrm{I} \text { and } \ell \leq-1 \quad \text { or } \quad h=3 \text { and } \ell \geq 1, \\ G_{h}\left(\rho_{-\frac{1}{2}}^{h, s}, \hat{\rho}_{h}\right) & \text { if } h \in \mathrm{I} \text { and } \ell=0 \\ G_{h}\left(\check{\rho}_{3}, \rho_{\frac{1}{2}}^{h, s}\right) & \text { if } h=3 \text { and } \ell=0\end{cases}
$$

and $F_{h}\left(\rho_{\ell-1 / 2}^{h, s}, \rho_{\ell+1 / 2}^{h, s}\right)$ is a monotone, consistent numerical flux, i.e. for all $h \in \mathrm{H}$

- $F_{h}$ is Lipschitz continuous from $\left[0, \rho_{\max }\right]^{2}$ to $\mathbb{R}$,

$-F_{h}(a, a)=f_{h}(a)$ for any $a \in\left[0, \rho_{\max }\right]$,

- the map $(a, b) \in\left[0, \rho_{\text {max }}\right]^{2} \mapsto F_{h}(a, b) \in \mathbb{R}$ is non-decreasing with respect to $a$ and non-increasing with respect to $b$.

In principle any monotone and consistent numerical flux might be used away from the junction, but we limit our attention to Godunov flux.

Notice that the choice $\vec{\rho}$ of the implementation of the local or non-local point constraint happens when we compute the boundary data in (5.1). In particular, when we deal with the non-local point constraint, we need to approximate the weighted average of the density $\zeta_{i}, i \in \mathrm{I}$ as follows

$$
Z_{i}^{s}=\Delta x \sum_{\ell \leq 0} w_{i}\left(x_{\ell+\frac{1}{2}}\right) \rho_{\ell+\frac{1}{2}}^{i, s}, \quad i \in \mathrm{I}
$$

Moreover, when we implement the local point constraint, we can apply Proposition 3.1 in order to find the boundary data in (5.1) corresponding to $\mathcal{R}_{j}^{l}$. However, in our simulations we implement $\mathcal{R}_{j}^{\mathrm{HBC}}$, indeed, as already observed, after few time iterations we observe the solution corresponding to $\mathcal{R}_{j}^{l}$.

\subsection{Validation of the numerical scheme}

The implementation of the scheme described in [1] for a merge without capacity drop representation has been done in [26]. 

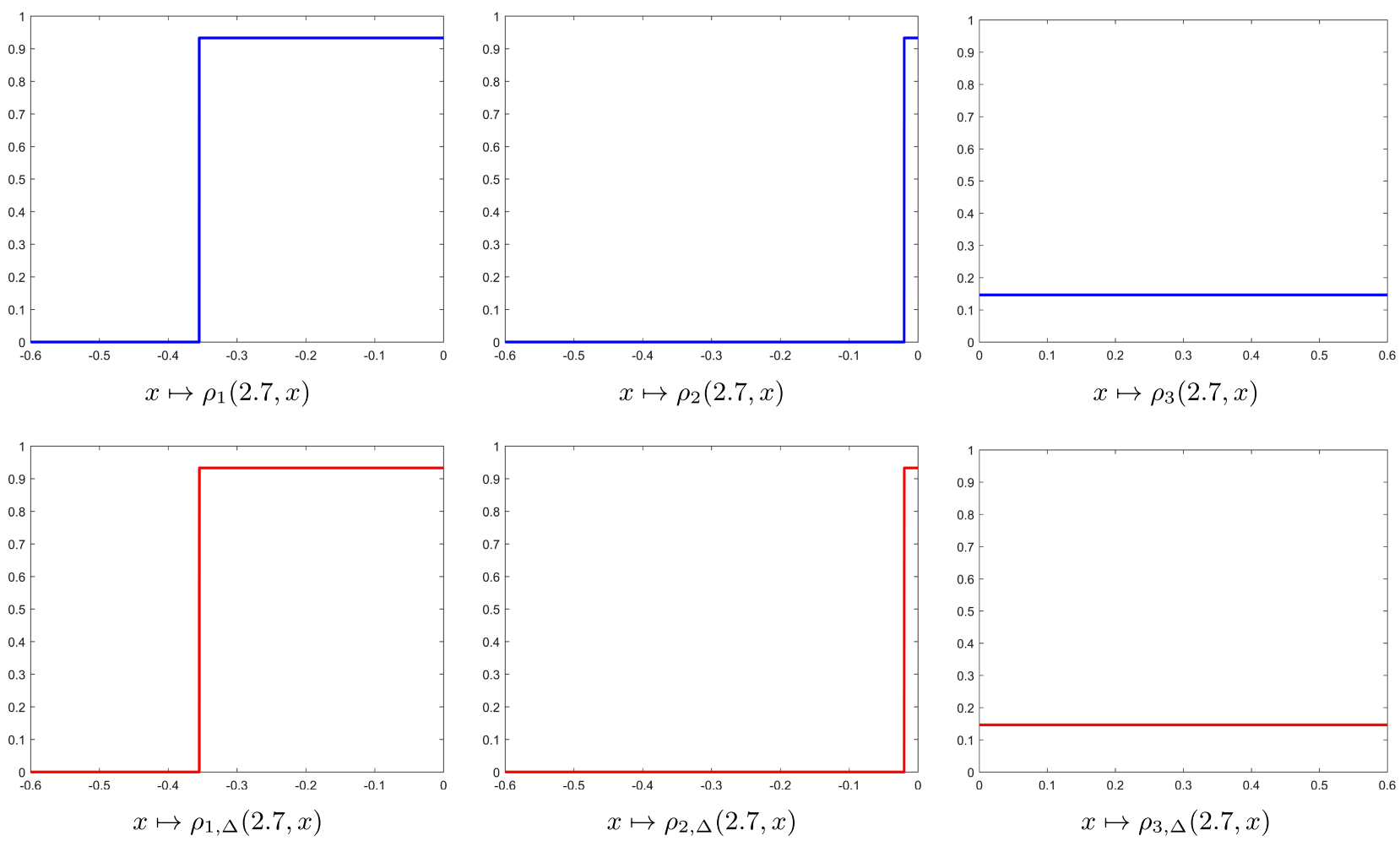

FiguRE 5. With reference to the simulation of Section 5.2.1, the comparison between the explicit solution $\vec{\rho}$ and the numerical one $\vec{\rho}_{\Delta}$ at time $t=2.7$.

\subsubsection{Validation of the implementation of the local point constraint}

In this section we validate the scheme subject to a local point constraint. In Figure 5, we can observe a good agreement between the explicit solution described in Section 3.1 and its numerical approximation at time $t=2.7$. For the simulation, we fix $[-3 / 5,0]$ as domain of computation for the incoming roads and $[0,3 / 5]$ for the outgoing one, and $\Delta x=10^{-4}, \Delta t=0.25 \times 10^{-4}$ as space step and time step, respectively.

Additionally, we perform a convergence analysis for this test. We introduce the relative $\mathrm{L}^{1}$-error respectively for the whole network, for the incoming and for the outgoing roads at a given time $t^{s}$ as follows

$$
E_{\mathrm{L}^{1}}^{s, \mathcal{N}}=\frac{\sum_{h=1}^{3} \sum_{\ell}\left|\rho_{h}\left(t^{s}, x_{\ell}\right)-\rho_{\ell}^{h, s}\right|}{\sum_{h=1}^{3} \sum_{\ell}\left|\rho_{h}\left(t^{s}, x_{\ell}\right)\right|}, \quad E_{\mathrm{L}^{1}}^{s, \mathrm{I}}=\frac{\sum_{i=1}^{2} \sum_{\ell}\left|\rho_{i}\left(t^{s}, x_{\ell}\right)-\rho_{\ell}^{i, s}\right|}{\sum_{i=1}^{2} \sum_{\ell}\left|\rho_{i}\left(t^{s}, x_{\ell}\right)\right|}, \quad E_{\mathrm{L}^{1}}^{s, 3}=\frac{\sum_{\ell}\left|\rho_{3}\left(t^{s}, x_{\ell}\right)-\rho_{\ell}^{3, s}\right|}{\sum_{\ell}\left|\rho_{3}\left(t^{s}, x_{\ell}\right)\right|} .
$$

Table 2 depicts the relative $\mathrm{L}^{1}$-error with respect to the space step at the fixed time $t=2.7$. The time step is fixed to $\Delta t=0.25 \times 10^{-4}$. We can easily observe that the rate of convergence is approximately 1 . This means that the introduction of a local point constraint does not affect the accuracy of the scheme. We stress that the rate of convergence related to $E_{\mathrm{L}^{1}}^{s, 3}$ is not significant in this case. In fact, due to the choice of the domain of computation for $\Omega_{3}$, we are computing the $\mathrm{L}^{1}$-error between two constant values, therefore the error has about the order of the machine accuracy and the rate of convergence presents some oscillations before stabilizing around 1 .

\subsubsection{Validation of the implementation of the non-local point constraint}

In order to show that the numerical scheme (5.1)-(5.3) is able to integrate in a coherent way the non-local point constraint at the junction, we validate it by comparison with the explicit solution computed in Section 4.1. 
TABLE 2. Relative $\mathrm{L}^{1}$-error at time $t=2.7$ computed in Section 5.2.1.

\begin{tabular}{llllll}
\hline \hline $\begin{array}{l}\text { Number } \\
\text { of cells } \\
\text { per road }\end{array}$ & $E_{\mathrm{L}^{1}}^{s, \mathcal{N}}$ & $\begin{array}{l}\text { Rate of } \\
\text { convergence }\end{array}$ & $E_{\mathrm{L}^{1}}^{s, \mathrm{l}}$ & $\begin{array}{l}\text { Rate of } \\
\text { convergence }\end{array}$ & $E_{\mathrm{L}^{1}}^{s, 3}$ \\
\hline 60 & $2.9607 \times 10^{-2}$ & - & $3.7143 \times 10^{-2}$ & - & $1.6320 \times 10^{-12}$ \\
120 & $1.9960 \times 10^{-2}$ & 0.5689 & $2.4973 \times 10^{-2}$ & 0.5728 & $1.6128 \times 10^{-12}$ \\
600 & $3.9689 \times 10^{-3}$ & 0.8958 & $4.9656 \times 10^{-3}$ & 0.8967 & $1.5943 \times 10^{-12}$ \\
1200 & $1.9700 \times 10^{-3}$ & 0.9268 & $2.4648 \times 10^{-3}$ & 0.9275 & $1.5728 \times 10^{-12}$ \\
6000 & $3.7094 \times 10^{-4}$ & 0.9675 & $4.6409 \times 10^{-4}$ & 0.9680 & $1.3055 \times 10^{-12}$ \\
12000 & $2.7758 \times 10^{-4}$ & 0.9307 & $3.4728 \times 10^{-4}$ & 0.9310 & $7.5801 \times 10^{-16}$ \\
\hline
\end{tabular}
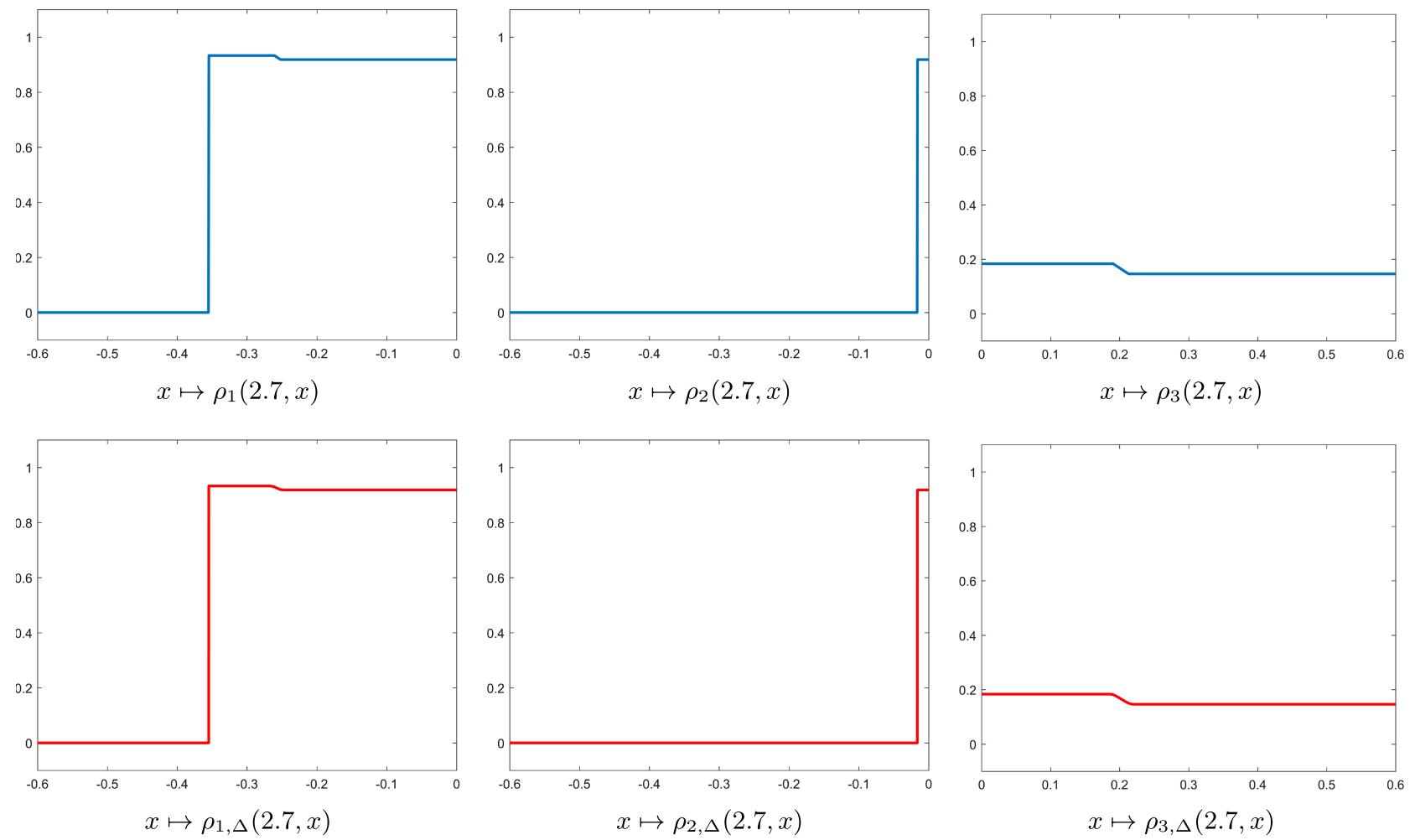

FiguRE 6. With reference to the simulation of Section 5.2.2, the comparison between the explicit solution $\vec{\rho}$ and the numerical one $\vec{\rho}_{\Delta}$ at time $t=2.7$.

For the simulation, we take the space step $\Delta x=0.5 \times 10^{-4}$ and the time step $\Delta t=0.25 \times 10^{-4}$.

In Figure 6 we compare the numerical and the explicit solution at time $t=2.7$. We observe a good agreement of these profiles. A convergence analysis is performed also for this test.

Table 3 shows the relative $\mathrm{L}^{1}$-error with respect to the space step at the fixed time $t=2.7$. The time step is fixed to $\Delta t=0.25 \times 10^{-4}$. We can easily observe that the rate of convergence is approximately 1 . This means that the introduction of a non-local point constraint does not affect the accuracy of the scheme. 
TABLE 3. Relative $\mathrm{L}^{1}$-error at time $t=2.7$ computed in Section 5.2.2.

\begin{tabular}{lllllll}
\hline \hline $\begin{array}{l}\text { Number } \\
\text { of cells } \\
\text { per road }\end{array}$ & $E_{\mathrm{L}^{1}}^{s, \mathcal{N}}$ & $\begin{array}{l}\text { Rate of } \\
\text { convergence }\end{array}$ & $E_{\mathrm{L}^{1}}^{s, l}$ & $\begin{array}{l}\text { Rate of } \\
\text { convergence }\end{array}$ & $E_{\mathrm{L}^{1}}^{s, 3}$ & $\begin{array}{l}\text { Rate of } \\
\text { convergence }\end{array}$ \\
\hline 60 & $3.0860 \times 10^{-2}$ & - & $3.0882 \times 10^{-2}$ & - & $3.0783 \times 10^{-2}$ & - \\
120 & $2.4713 \times 10^{-2}$ & 0.3205 & $2.7028 \times 10^{-2}$ & 0.1923 & $1.6441 \times 10^{-2}$ & 0.9048 \\
600 & $5.4311 \times 10^{-3}$ & 0.7874 & $5.6482 \times 10^{-3}$ & 0.7792 & $4.6523 \times 10^{-3}$ & 0.8143 \\
1200 & $2.6768 \times 10^{-3}$ & 0.8442 & $2.6246 \times 10^{-3}$ & 0.8565 & $2.8639 \times 10^{-3}$ & 0.7909 \\
6000 & $9.3434 \times 10^{-4}$ & 0.8024 & $9.4064 \times 10^{-4}$ & 0.8115 & $9.1174 \times 10^{-4}$ & 0.7612 \\
12000 & $5.8915 \times 10^{-4}$ & 0.7816 & $6.0521 \times 10^{-4}$ & 0.7867 & $5.3150 \times 10^{-4}$ & 0.7560 \\
\hline
\end{tabular}
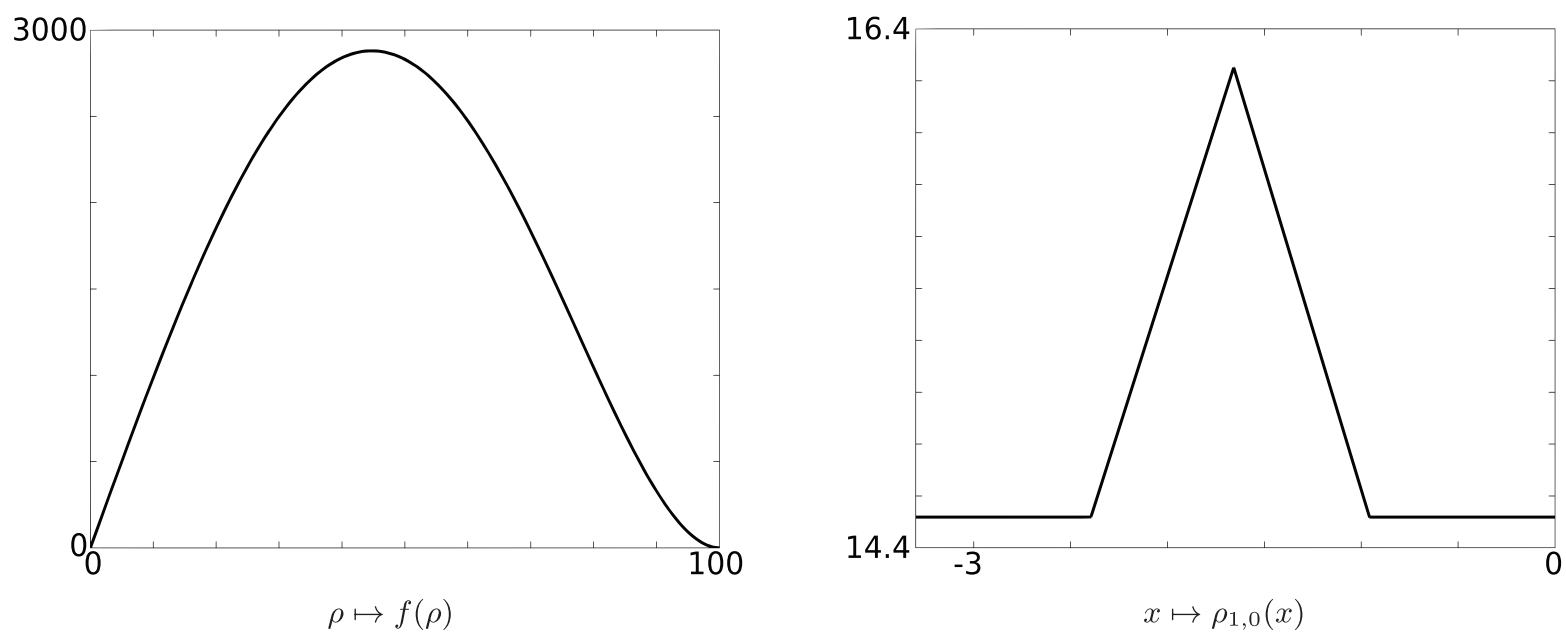

FiguRE 7. Flux and initial condition on the first incoming road considered in Section 5.3.

\subsection{Local and non-local constraints at the junction}

In this section, we perform a simulation analogous to the one made in [22]. We model the incoming roads by the segment $[-12 / 5,0]$ and the outgoing road by $[0,12 / 5]$. We take

$$
f(\rho)=\frac{9}{4 \cdot 10^{6} \sqrt{5}} \rho\left(10^{4}-\rho^{2}\right)^{2},
$$

as flux for each road, see Figure 7 on the left. As initial conditions, see Figure 7 on the right, we use

$$
\rho_{1,0}(x)=\left\{\begin{array}{lll}
p_{1} & \text { if } x \leq x_{1}, & \\
p_{1}+\frac{p_{2}-p_{1}}{x_{2}-x_{1}}\left(x-x_{1}\right) & \text { if } x_{1}<x \leq x_{2}, & \\
p_{1}-\frac{p_{2}-p_{1}}{x_{3}-x_{2}}\left(x-x_{3}\right) & \text { if } x_{2}<x \leq x_{3}, & \rho_{2,0}=p_{1},
\end{array} \quad \rho_{3,0}=p_{3},\right.
$$

where

$$
p_{1}=14.5190, \quad p_{2}=16.2511, \quad p_{3}=38.0366, \quad x_{1}=-2.3957, \quad x_{2}=-1.6588, \quad x_{3}=-0.9583 .
$$



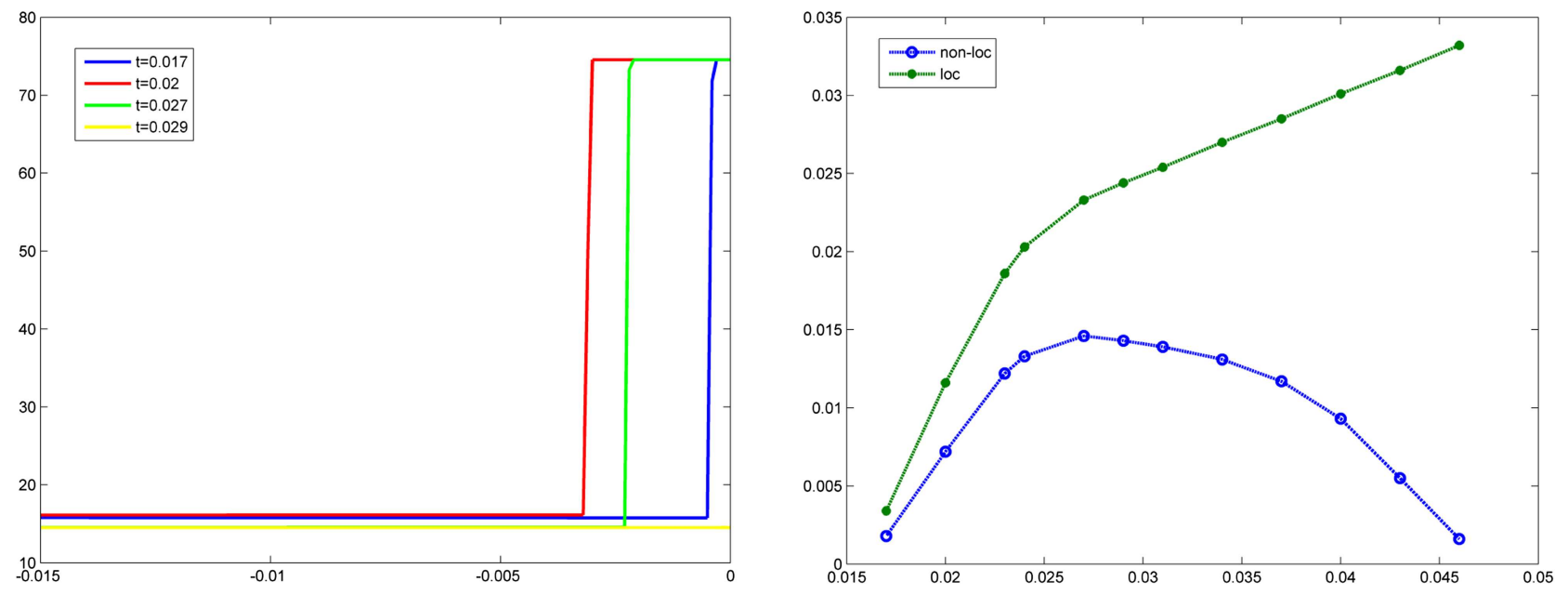

FIGURE 8. Left panel: profiles of solution on $\Omega_{1}$ corresponding to $\mathcal{R}_{j}^{\mathrm{CGP}}$. Right panel: dynamic of the shocks on $\Omega_{1}$ in the solution corresponding to the local model $\mathcal{R}_{j}^{l}$ and the non-local model $\mathcal{S}_{j}^{\text {nl }}$. On the $x$-axis we represent time and on the $y$-axis we represent the distance from the junction.

As constraint function we use

$$
g(s)= \begin{cases}2880 & \text { if } 0 \leq s \leq 2880 \\ 5760-s & \text { if } 2880<s<3024 \\ 2736 & \text { if } 3024 \leq s \leq 5760\end{cases}
$$

and we choose the weight function $w(x)=2(1+x) \chi_{[-1,0]}(x)$. We take the space step $\Delta x=10^{-4}$, the time step $\Delta t=(\sqrt{5} / 45) \times 10^{-5}$ and the priority factor $\alpha=1 / 2$. We compute and compare the approximate solutions corresponding to $\mathcal{R}_{j}^{\mathrm{CGP}}, \mathcal{R}_{j}^{l}$ and $\mathcal{S}_{j}^{\mathrm{nl}}$.

Figure 8 shows that, as observed in [22], even if the initial condition leads to a moderate congestion which quickly disappears in the solution corresponding to $\mathcal{R}_{j}^{C G P}$, the shock in the solution corresponding to $\mathcal{R}_{j}^{l}$ is never reabsorbed and the congestion keeps growing forever. On the other hand, the non-local model $\mathcal{S}_{j}^{\text {nl }}$ reproduces a more realistic behavior, at least in these cases, as the congestion is reabsorbed in finite time and at a smoothly increasing rate.

\subsubsection{Qualitative behavior of the numerical solutions of the non-local model depending on the observation interval}

A natural question on our nonlocal model concerns the role of the weight function $w$. To clarify the impact of $w$ on the qualitative behavior of the solution we run simulations using the same setting and data as in the previous section, but letting the support of $w$ vary. Even if this study does not provide a precise insight of the general case, it gives some hints on how the calibration of such parameter can be done. We observed that the qualitative behavior of the solution changes continuously depending on the value of $\min \{\operatorname{supp}(w)\}$ and that only a very small range of values gives the desired dynamics where we can observe capacity drop and capacity recovery. We performed simulations for values of $\min \{\operatorname{supp}(w)\}$ between -0.9 and -1.3 , and we noticed that

- if the support of $w$ is too small (essentially $\min \{\operatorname{supp}(w)\} \geq-0.95$ ) then the numerically computed solution is very close to the solution obtained in the locally constrained setting on all of the branches, in particular we do not observe any reduction of the congestion; 

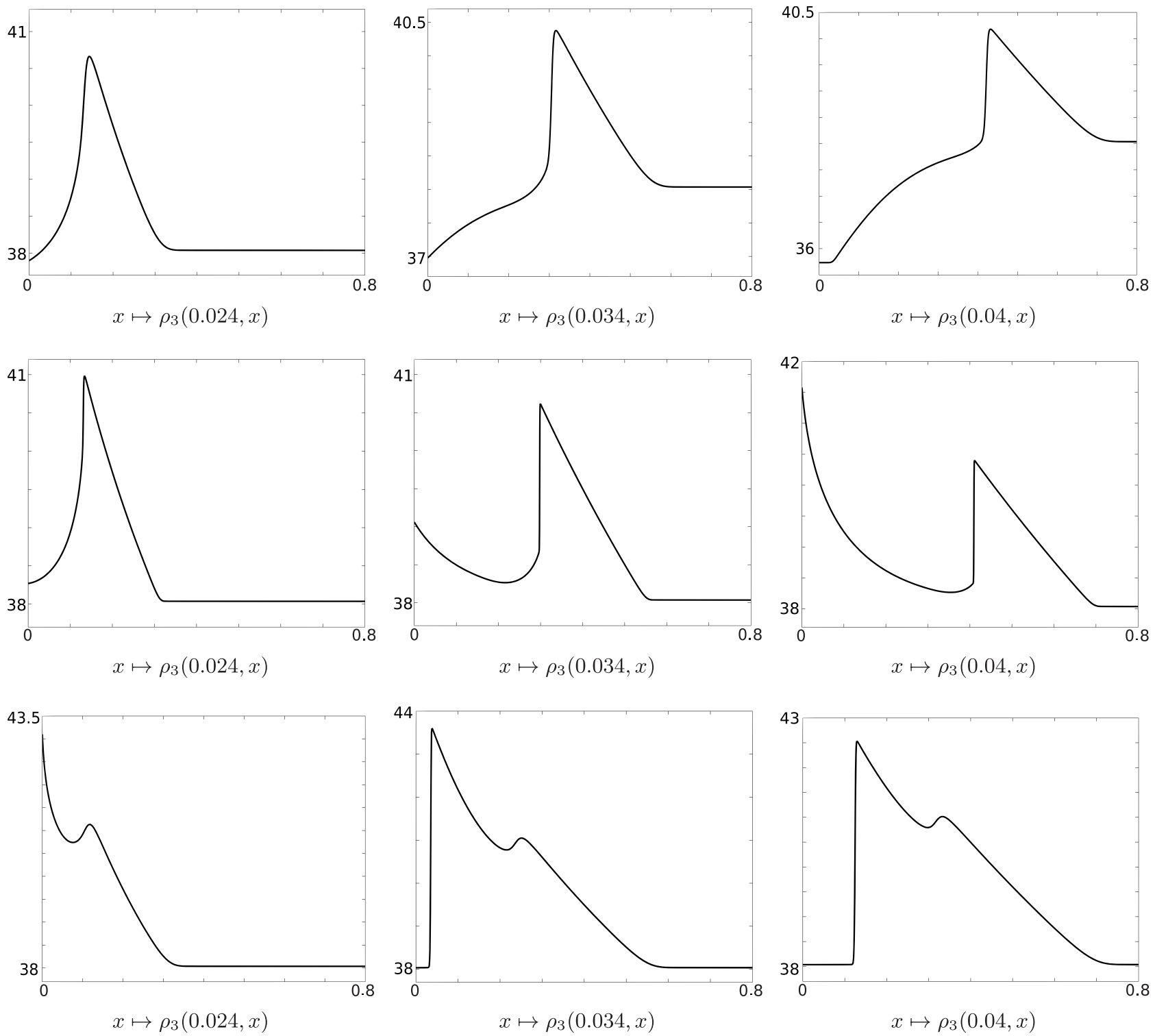

Figure 9. With reference to Section 5.3.1, the profiles of the solution on $\Omega_{3}$ with varying observation interval $\operatorname{supp}(w)$ : in the first line $\operatorname{supp}(w)=[-0.98,0]$, in the second line $\operatorname{supp}(w)=$ $[-1,0]$, in the last line $\operatorname{supp}(w)=[-1.2,0]$.

- if $\min \{\operatorname{supp}(w)\} \in[-0.98,-0.95)$, the capacity of the junction diminishes more gradually, but even in this case the congestion last forever as in the local model;

- if $\min \{\operatorname{supp}(w)\} \in[-1.2,-0.98$ ), we observe a capacity recovery (which might be interpreted as "self organization") shortly after the capacity drop, this is the most interesting situation;

- if $\min \{\operatorname{supp}(w)\}<-1.2$, the capacity drop becomes so small that it practically does not have any impact on the behavior of solutions. This is due to the fact that while the support of $w$ increases, its $\mathrm{L}^{1}$-norm is constantly equal to 1 , hence the perturbation of the data on $\Omega_{1}$ becomes negligible.

We plot in Figure 9 the profiles of the solutions on $\Omega_{3}$ corresponding to $\min \{\operatorname{supp}(w)\} \in\{-1.2,-1,-0.98\}$. 


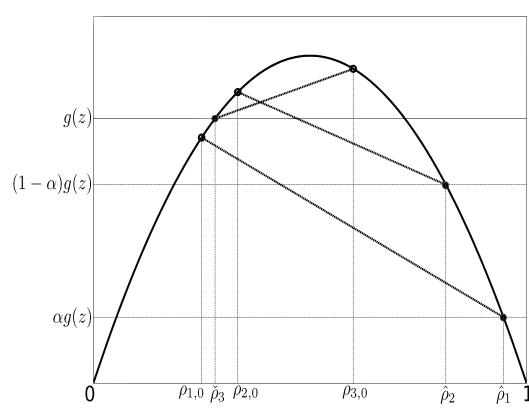

(A) The waves joining initial conditions and boundary values on each of the roads.

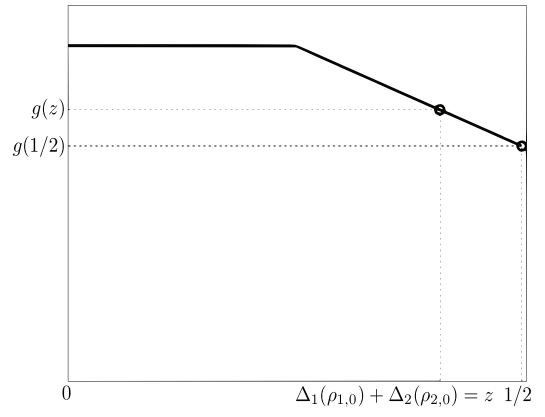

(B) Two different levels of the constraint $g$.

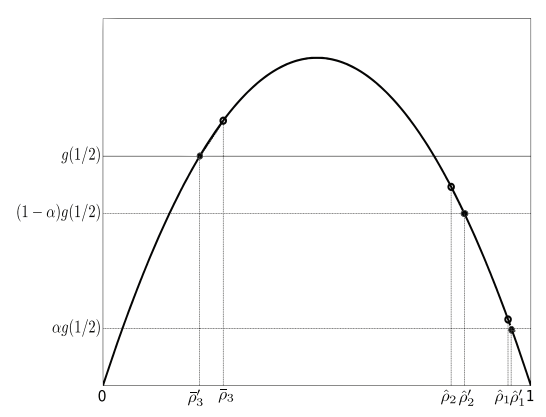

(C) The traces of the solution after a new resolution of the Riemann problem.

Figure 10. With reference to Section 6.1: The changing of the trace of solutions and the levels of the constraint $g$.

\section{TECHNICAL SECTION}

In this section we prove Theorem 2.11. We recall that the properties of $\mathcal{R}_{j}^{\text {CGP }}$ are already proved in $[18,21]$. We prove the non-consistency of $\mathcal{R}_{j}^{\mathrm{HBC}}$ in Section 6.1, the lack of $\mathrm{L}_{\text {loc }}^{1}$-continuity for $\mathcal{R}_{j}^{l}$ in Section 6.2 , and the consistency of $\mathcal{R}_{j}^{l}$ in Section 6.3. The property (P1) follows from the case studies in Section 6.3. The lack of self-similarity for $\mathcal{S}_{j}^{\text {nl }}$ is already clear from the exact solution in Section 4.1.

\subsection{Non-consistency of $\mathcal{R}_{j}^{\mathrm{HBC}}$}

In this section we give an explicit example to show that in general $\mathcal{R}_{j}^{\mathrm{HBC}}$ is not consistent. We take the priority factor $\alpha=1 / 4$, the flux $f(\rho)=\rho(1-\rho)$ on every road, that is $f_{h} \equiv f, h \in \mathrm{H}$, the constraint

$$
g(s)= \begin{cases}\frac{1}{4} & \text { if } 0 \leq s \leq \frac{1}{4}, \\ \frac{13}{40}-\frac{3}{10} s & \text { if } \frac{1}{4}<s \leq \frac{1}{2},\end{cases}
$$

and the initial condition $\vec{\rho}_{0}=(1 / 4,1 / 3,3 / 5)$, see Figure 10 .

Clearly $f_{h}^{\max }=f^{\max }=1 / 4$. In this case $\vec{\rho}_{0} \in \mathrm{BBB}$ and

$$
\begin{array}{rlrl}
\Delta_{1,0} & =\Delta_{1}\left(\rho_{1,0}\right)=f\left(\rho_{1,0}\right)<f^{\max }, & \Delta_{1,0}+\Delta_{2,0}>\Sigma_{3,0}>g\left(\Delta_{1,0}+\Delta_{2,0}\right), \\
\Delta_{2,0}=\Delta_{2}\left(\rho_{2,0}\right) & =f\left(\rho_{2,0}\right)<f^{\max }, & \alpha g\left(\Delta_{1,0}+\Delta_{2,0}\right) & <\Delta_{1,0}, \\
\Sigma_{3,0} & =\Sigma_{3}\left(\rho_{3,0}\right)=f\left(\rho_{3,0}\right)<f^{\max }, & (1-\alpha) g\left(\Delta_{1,0}+\Delta_{2,0}\right) & <\Delta_{2,0},
\end{array}
$$

hence by $(2.12)$ and $(6.1)_{2}$ we have

$$
Q^{\mathrm{HBC}}\left(\vec{\rho}_{0}\right)=g\left(\Delta_{1,0}+\Delta_{2,0}\right) \approx 0.2021<\Delta_{1,0}+\Delta_{2,0} \approx 0.4097 .
$$

As a consequence the passing flow at the junction is $Q^{\mathrm{HBC}}\left(\vec{\rho}_{0}\right)$. We determine now the passing flow coming from each of the incoming roads. By $(6.2)_{2}$ and $(6.3)_{2}$ we have

$$
Q^{\mathrm{HBC}}\left(\vec{\rho}_{0}\right)-\Delta_{2,0}<\alpha Q^{\mathrm{HBC}}\left(\vec{\rho}_{0}\right)<\Delta_{1,0} .
$$

Since $\vec{\rho} \equiv \mathcal{R}_{j}^{\mathrm{HBC}}\left[\vec{\rho}_{0}\right]$ has the form (2.4)-(2.5) with $Q \equiv Q^{\mathrm{HBC}}$, we have $\Gamma_{1}=\alpha Q^{\mathrm{HBC}}\left(\vec{\rho}_{0}\right)$ and $\Gamma_{2}=(1-$

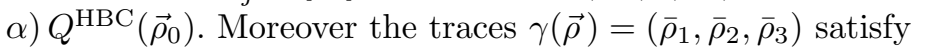

$$
\bar{\rho}_{1}=\hat{\rho}_{1}\left(\alpha Q^{\mathrm{HBC}}\left(\vec{\rho}_{0}\right)\right) \approx 0.9466, \quad \bar{\rho}_{2}=\hat{\rho}_{2}\left((1-\alpha) Q^{\mathrm{HBC}}\left(\vec{\rho}_{0}\right)\right) \approx 0.8137, \quad \bar{\rho}_{3}=\check{\rho}_{3}\left(Q^{\mathrm{HBC}}\left(\vec{\rho}_{0}\right)\right) \approx 0.2811 .
$$


We prove now that the traces $\gamma(\vec{\rho})$ are not an equilibrium for $\mathcal{R}_{j}^{\mathrm{HBC}}$ and, as a consequence, that $\mathcal{R}_{j}^{\mathrm{HBC}}$ is not consistent. By (6.4) we have $\gamma(\vec{\rho}) \in \mathrm{GGG}$ and therefore

$$
\Delta_{1}\left(\bar{\rho}_{1}\right)=\Delta_{2}\left(\bar{\rho}_{2}\right)=\Sigma_{3}\left(\bar{\rho}_{3}\right)=f^{\max }=1 / 4 .
$$

We have then

$$
Q^{\mathrm{HBC}}(\gamma(\vec{\rho}))=g\left(2 f^{\max }\right)=g_{\min }=7 / 40,
$$

because the monotonicity of $g$ and $(6.1)_{2}$ imply

$$
g_{\min }=g\left(\Delta_{1}\left(\bar{\rho}_{1}\right)+\Delta_{2}\left(\bar{\rho}_{2}\right)\right)<g\left(\Delta_{1,0}+\Delta_{2,0}\right)<\Sigma_{3,0}<f^{\max }=\Sigma_{3}\left(\bar{\rho}_{3}\right) .
$$

Clearly

$$
\begin{gathered}
Q^{\mathrm{HBC}}(\gamma(\vec{\rho}))=7 / 40<\Delta_{1}\left(\bar{\rho}_{1}\right)+\Delta_{2}\left(\bar{\rho}_{2}\right)=1 / 2, \\
Q^{\mathrm{HBC}}(\gamma(\vec{\rho}))-\Delta_{2}\left(\bar{\rho}_{2}\right)=-3 / 40<\alpha Q^{\mathrm{HBC}}(\gamma(\vec{\rho}))=7 / 160<\Delta_{1}\left(\bar{\rho}_{1}\right)=1 / 4,
\end{gathered}
$$

and by $(2.5)$ we have

$$
\Gamma_{1}^{\mathrm{HBC}}(\gamma(\vec{\rho}))=\alpha Q^{\mathrm{HBC}}(\gamma(\vec{\rho})), \quad \quad \Gamma_{2}^{\mathrm{HBC}}(\gamma(\vec{\rho}))=(1-\alpha) Q^{\mathrm{HBC}}(\gamma(\vec{\rho})) .
$$

Since $Q^{\mathrm{HBC}}(\gamma(\vec{\rho}))<Q^{\mathrm{HBC}}\left(\vec{\rho}_{0}\right)$, by the definitions of $\hat{\rho}_{i}, i \in \mathrm{I}$, we have that $\gamma(\vec{\rho})$ is not an equilibrium for $\mathcal{R}_{j}^{\mathrm{HBC}}$, namely $\mathcal{R}_{j}^{\mathrm{HBC}}[\gamma(\vec{\rho})] \not \equiv \gamma(\vec{\rho})$. Indeed, by (6.4) we have the estimates

$$
\begin{aligned}
f_{1}\left(\bar{\rho}_{1}\right) & =f_{1}\left(\hat{\rho}_{1}\left(\alpha Q^{\mathrm{HBC}}\left(\vec{\rho}_{0}\right)\right)\right)=\alpha Q^{\mathrm{HBC}}\left(\vec{\rho}_{0}\right)>\alpha Q^{\mathrm{HBC}}(\gamma(\vec{\rho}))=f_{1}\left(\hat{\rho}_{1}\left(\alpha Q^{\mathrm{HBC}}(\gamma(\vec{\rho}))\right)\right), \\
f_{2}\left(\bar{\rho}_{2}\right) & =f_{2}\left(\hat{\rho}_{2}\left((1-\alpha) Q^{\mathrm{HBC}}\left(\vec{\rho}_{0}\right)\right)\right)=(1-\alpha) Q^{\mathrm{HBC}}\left(\vec{\rho}_{0}\right)>(1-\alpha) Q^{\mathrm{HBC}}(\gamma(\vec{\rho})) \\
& =f_{2}\left(\hat{\rho}_{2}\left((1-\alpha) Q^{\mathrm{HBC}}(\gamma(\vec{\rho}))\right)\right) \\
f_{3}\left(\bar{\rho}_{3}\right) & =f_{3}\left(\check{\rho}_{3}\left(Q^{\mathrm{HBC}}\left(\vec{\rho}_{0}\right)\right)\right)=Q^{\mathrm{HBC}}\left(\vec{\rho}_{0}\right)>Q^{\mathrm{HBC}}(\gamma(\vec{\rho}))=f_{3}\left(\check{\rho}_{3}\left(Q^{\mathrm{HBC}}(\gamma(\vec{\rho}))\right)\right),
\end{aligned}
$$

which imply that $\mathcal{R}_{j}^{\mathrm{HBC}}[\gamma(\vec{\rho})]$ has one shock on each road and therefore

$$
\gamma\left(\mathcal{R}_{j}^{\mathrm{HBC}}[\gamma(\vec{\rho})]\right)=\left(\hat{\rho}_{1}\left(\alpha Q^{\mathrm{HBC}}(\gamma(\vec{\rho}))\right), \hat{\rho}_{2}\left((1-\alpha) Q^{\mathrm{HBC}}(\gamma(\vec{\rho}))\right), \check{\rho}_{3}\left(Q^{\mathrm{HBC}}(\gamma(\vec{\rho}))\right)\right) \neq \gamma(\vec{\rho}) .
$$

\subsection{Lack of $\mathrm{L}_{\text {loc }}^{1}$-continuity for $\mathcal{R}_{j}^{l}$}

In this section we give an example to show that $\mathcal{R}_{j}^{l}$ is not $\mathrm{L}_{\text {loc }}^{1}$-continuous in general. Let $M>0$, large enough, $\alpha \in[0,1], g$ a constraint function and consider $\vec{\rho}_{0} \in$ BBG such that $f_{1}\left(\rho_{1,0}\right)=\alpha f_{3}^{\max }, f_{2}\left(\rho_{2,0}\right)=(1-\alpha) f_{3}^{\max }$ and $\rho_{3,0}=0$. We have

$$
\Delta_{1}\left(\rho_{1,0}\right)+\Delta_{2}\left(\rho_{2,0}\right)=f_{1}\left(\rho_{1,0}\right)+f_{2}\left(\rho_{2,0}\right)=f_{3}^{\max },
$$

then $Q^{l}\left(\vec{\rho}_{0}\right)=f_{3}^{\max }$ and $\mathcal{T}\left[\vec{\rho}_{0}\right]=\left(\rho_{1,0}, \rho_{2,0}, \rho_{3, c}\right)$.

Fix $\varepsilon>0$ such that $\vec{\rho}_{0, \varepsilon}=\left(\rho_{1, \varepsilon}, \rho_{2, \varepsilon}, \rho_{3, \varepsilon}\right)=\left(\rho_{1,0}+\varepsilon, \rho_{2,0}+\varepsilon, 0\right) \in$ BBG. Clearly

$$
\Delta_{1}\left(\rho_{1, \varepsilon}\right)+\Delta_{2}\left(\rho_{2, \varepsilon}\right)=f_{1}\left(\rho_{1, \varepsilon}\right)+f_{2}\left(\rho_{2, \varepsilon}\right)>f_{3}^{\max }, \quad Q^{\mathrm{HBC}}\left(\vec{\rho}_{0, \varepsilon}\right)=g\left(f_{1}\left(\rho_{1, \varepsilon}\right)+f_{2}\left(\rho_{2, \varepsilon}\right)\right)=g_{\varepsilon},
$$

and

$$
f_{1}\left(\rho_{1, \varepsilon}\right)>f_{1}\left(\rho_{1,0}\right)=\alpha f_{3}^{\max }>\alpha g_{\varepsilon}>\alpha g_{\min }, \quad f_{2}\left(\rho_{2, \varepsilon}\right)>f_{2}\left(\rho_{2,0}\right)=(1-\alpha) f_{3}^{\max }>(1-\alpha) g_{\varepsilon}>(1-\alpha) g_{\min },
$$

therefore, $Q^{l}\left(\vec{\rho}_{0, \varepsilon}\right)=g_{\text {min }}$ and $\mathcal{T}^{3}\left[\vec{\rho}_{0, \varepsilon}\right]=\left(\hat{\rho}_{1, \alpha}, \hat{\rho}_{2, \alpha}, \check{\rho}_{3, g}\right)$, see Section 6.3 case (BBG.ii-2). This means that the resulting waves are shocks with negative speeds $\sigma_{i}$, on $\Omega_{i}, i \in \mathrm{I}$, and a rarefaction with positive speed on $\Omega_{3}$. 
Therefore, for $t$ sufficiently small, if we compute the $\mathrm{L}_{\text {loc }}^{1}$-norm of the difference of solutions, we obtain

$$
\begin{aligned}
& \int_{-M}^{0}\left(\left|\rho_{1, \varepsilon} \chi_{\left(-\infty, \sigma_{1} t\right]}(x)+\hat{\rho}_{1, \alpha} \chi_{\left[\sigma_{1} t, 0\right]}(x)-\rho_{1,0}\right|+\left|\rho_{2, \varepsilon} \chi_{\left(-\infty, \sigma_{2} t\right]}(x)+\hat{\rho}_{2, \alpha} \chi_{\left[\sigma_{2} t, 0\right]}(x)-\rho_{2,0}\right|\right) \mathrm{d} x \\
& +\int_{0}^{M}\left(\left|\check{\rho}_{3, g} \chi_{\left[0, f_{3}^{\prime}\left(\check{\rho}_{3, g}\right) t\right]}(x)+\left(f_{3}^{\prime}\right)^{-1}(x / t) \chi_{\left[f_{3}^{\prime}\left(\check{\rho}_{3, g}\right) t, f_{3}^{\prime}(0) t\right]}(x)-\left(f_{3}^{\prime}\right)^{-1}(x / t) \chi_{\left[0, f_{3}^{\prime}(0) t\right]}\right|(x)\right) \mathrm{d} x \\
& \geq \varepsilon\left(\left|\sigma_{1} t+M\right|+\left|\sigma_{2} t+M\right|\right)+\underbrace{\left(f_{1}\left(\rho_{1, \varepsilon}\right)-\alpha g_{\min }\right)}_{>\alpha\left(f_{3}^{\max }-g_{\min }\right)} \frac{\hat{\rho}_{1, \alpha}-\rho_{1,0}}{\left|\hat{\rho}_{1, \alpha}-\rho_{1,0}-\varepsilon\right|} t \\
& +\underbrace{\left(f_{2}\left(\rho_{2, \varepsilon}\right)-(1-\alpha) g_{\min }\right)}_{>(1-\alpha)\left(f_{3}^{\max }-g_{\min }\right)} \frac{\hat{\rho}_{2, \alpha}-\rho_{2,0}}{\left|\hat{\rho}_{2, \alpha}-\rho_{2,0}-\varepsilon\right|} t+\underbrace{\int_{0}^{M}\left(\check{\rho}_{3, g}-\left(f_{3}^{\prime}\right)^{-1}(x / t)\right) \chi_{\left[0, f_{3}^{\prime}\left(\check{\rho}_{3, g}\right) t\right]}(x) \mathrm{d} x}_{>0} .
\end{aligned}
$$

Remark 6.1. We can observe that in the same setting as above, $\mathcal{R}_{j}^{\mathrm{HBC}}$ does not show a discontinuous behavior. Indeed we have $Q^{\mathrm{HBC}}=g_{\varepsilon}=g\left(f_{1}\left(\rho_{1, \varepsilon}\right)+f_{2}\left(\rho_{2, \varepsilon}\right)\right)$ and $\mathcal{T}\left[\vec{\rho}_{0}\right]=\left(\hat{\rho}_{1}\left(\alpha g_{\varepsilon}\right), \hat{\rho}_{2}\left((1-\alpha) g_{\varepsilon}\right), \check{\rho}_{3}\left(g_{\varepsilon}\right)\right)$, so the resulting waves are shocks with negative speeds $\tilde{\sigma}_{i}$, on $\Omega_{i}, i \in \mathrm{I}$, and a rarefaction with positive speed on $\Omega_{3}$. Notice that, if we set $L=\max \left\{\operatorname{Lip}\left(f_{1}\right), \operatorname{Lip}\left(f_{2}\right)\right\}$, we have

$$
\begin{aligned}
\left|g_{\varepsilon}-f_{3}^{\max }\right| & \leq \operatorname{Lip}(g)\left(f_{1}\left(\rho_{1, \varepsilon}\right)+f_{2}\left(\rho_{2, \varepsilon}\right)-f_{1}\left(\rho_{1,0}\right)-f_{2}\left(\rho_{2,0}\right)\right) \leq 2 L \operatorname{Lip}(g) \varepsilon, \\
\left|f_{1}\left(\rho_{1, \varepsilon}\right)-\alpha g_{\varepsilon}\right| & \leq \operatorname{Lip}\left(f_{1}\right) \varepsilon+\alpha \operatorname{Lip}(g)\left(f_{1}\left(\rho_{1, \varepsilon}\right)+f_{2}\left(\rho_{2, \varepsilon}\right)-f_{1}\left(\rho_{1,0}\right)-f_{2}\left(\rho_{2,0}\right)\right) \leq \varepsilon L(1+2 \alpha \operatorname{Lip}(g)), \\
\left|f_{2}\left(\rho_{2, \varepsilon}\right)-(1-\alpha) g_{\varepsilon}\right| & \leq \operatorname{Lip}\left(f_{2}\right) \varepsilon+(1-\alpha) \operatorname{Lip}(g)\left(f_{1}\left(\rho_{1, \varepsilon}\right)+f_{2}\left(\rho_{2, \varepsilon}\right)-f_{1}\left(\rho_{1,0}\right)-f_{2}\left(\rho_{2,0}\right)\right) \\
& \leq \varepsilon L(1+2(1-\alpha) \operatorname{Lip}(g)) .
\end{aligned}
$$

Therefore, by (6.5)-(6.7) we conclude that

$$
\begin{aligned}
& \int_{-M}^{0}\left(\left|\rho_{1, \varepsilon} \chi_{\left(-\infty, \tilde{\sigma}_{1} t\right]}(x)+\hat{\rho}_{1}\left(\alpha g_{\varepsilon}\right) \chi_{\left[\tilde{\sigma}_{1} t, 0\right]}(x)-\rho_{1,0}\right|+\left|\rho_{2, \varepsilon} \chi_{\left(-\infty, \tilde{\sigma}_{2} t\right]}(x)+\hat{\rho}_{2}\left((1-\alpha) g_{\varepsilon}\right) \chi_{\left[\tilde{\sigma}_{2} t, 0\right]}(x)-\rho_{2,0}\right|\right) \mathrm{d} x \\
& +\int_{0}^{M}\left(\left|\check{\rho}_{3}\left(g_{\varepsilon}\right) \chi_{\left[0, f_{3}^{\prime}\left(\check{\rho}_{3}\left(g_{\varepsilon}\right)\right) t\right]}(x)+\left(f_{3}^{\prime}\right)^{-1}(x / t) \chi_{\left[f_{3}^{\prime}\left(\check{\rho}_{3}\left(g_{\varepsilon}\right)\right) t, f_{3}^{\prime}(0) t\right]}(x)-\left(f_{3}^{\prime}\right)^{-1}(x / t) \chi_{\left[0, f_{3}^{\prime}(0) t\right]}(x)\right|\right) \mathrm{d} x \\
& \leq \varepsilon\left(\left|\tilde{\sigma}_{1} t+M\right|+\left|\tilde{\sigma}_{2} t+M\right|\right)+\underbrace{\left(f_{1}\left(\rho_{1, \varepsilon}\right)-\alpha g_{\varepsilon}\right)}_{\rightarrow 0 \text { as } \varepsilon \rightarrow 0} \frac{\hat{\rho}_{1}\left(\alpha g_{\varepsilon}\right)-\rho_{1,0}}{\left|\hat{\rho}_{1}\left(\alpha g_{\varepsilon}\right)-\rho_{1,0}-\varepsilon\right|} t \\
& +\underbrace{\left(f_{2}\left(\rho_{2, \varepsilon}\right)-(1-\alpha) g_{\varepsilon}\right)}_{\rightarrow 0 \text { as } \varepsilon \rightarrow 0} \frac{\hat{\rho}_{2}\left((1-\alpha) g_{\varepsilon}\right)-\rho_{2,0}}{\left|\hat{\rho}_{2}\left((1-\alpha) g_{\varepsilon}\right)-\rho_{2,0}-\varepsilon\right|} t+\underbrace{\check{\rho}_{3}\left(g_{\varepsilon}\right) f_{3}^{\prime}\left(\check{\rho}_{3}\left(g_{\varepsilon}\right)\right) t}_{\rightarrow 0 \text { as } \varepsilon \rightarrow 0} .
\end{aligned}
$$

\subsection{Consistency of $\mathcal{R}_{j}^{l}$}

In this section we prove that $\mathcal{R}_{j}^{l}$ is consistent. We recall that $\mathcal{R}_{j}^{l}$ is defined by taking $Q \equiv Q^{l}$ in Definition 2.7, where $Q^{l}$ is given in (2.13). The proof consists in the following steps:

- For any $\vec{\rho}_{0} \in \Lambda$ the traces $\gamma\left(\vec{\rho}^{l}\right)=\left(\bar{\rho}_{1}^{l}, \bar{\rho}_{2}^{l}, \bar{\rho}_{3}^{l}\right)$ of $\vec{\rho}^{l}=\mathcal{R}_{j}^{l}\left[\vec{\rho}_{0}\right]$ satisfy the following equation

$$
\bar{\rho}_{h}^{l}=\tau_{h}^{3}, \quad h \in \mathrm{H},
$$

where $\left(\tau_{1}^{3}, \tau_{2}^{3}, \tau_{3}^{3}\right)=\mathcal{T}^{3}\left[\vec{\rho}_{0}\right]$.

- For any $\vec{\rho}_{0} \in \Lambda$ and $k \geq 4$ we have that $\mathcal{T}^{k}\left[\vec{\rho}_{0}\right]=\mathcal{T}^{3}\left[\vec{\rho}_{0}\right]$.

$-\mathcal{R}_{j}^{l}$ is consistent. 
The consistency of $\mathcal{R}_{j}^{l}$ immediately follows from the previous two steps, that are considered in the following proposition.

Proposition 6.2. Let $\alpha \in[0,1]$ be a priority factor and $g$ be a constraint function as in (2.11). For any $\vec{\rho}_{0} \in \Lambda$ we have that the traces $\gamma\left(\vec{\rho}^{l}\right)=\left(\bar{\rho}_{1}^{l}, \bar{\rho}_{2}^{l}, \bar{\rho}_{3}^{l}\right)$ of $\vec{\rho}^{l}=\mathcal{R}_{j}^{l}\left[\vec{\rho}_{0}\right]$ satisfy $(6.8)$, moreover $\mathcal{T}^{k}\left[\vec{\rho}_{0}\right]=\mathcal{T}^{3}\left[\vec{\rho}_{0}\right]$ for any $k \geq 4$.

Proof. By (2.9) we have

$$
\begin{array}{ll}
\left(f_{1}\left(\bar{\rho}_{1}^{l}\right), f_{2}\left(\bar{\rho}_{2}^{l}\right), f_{3}\left(\bar{\rho}_{3}^{l}\right)\right)=\left(\Delta_{1,0}, \Delta_{2,0}, \Delta_{1,0}+\Delta_{2,0}\right) & \text { if } \Delta_{1,0}+\Delta_{2,0} \leq Q^{l}, \\
\left(f_{1}\left(\bar{\rho}_{1}^{l}\right), f_{2}\left(\bar{\rho}_{2}^{l}\right), f_{3}\left(\bar{\rho}_{3}^{l}\right)\right)=\left(\Delta_{1,0}, Q^{l}-\Delta_{1,0}, Q^{l}\right) & \text { if } Q^{l}-\Delta_{2,0}<\Delta_{1,0} \leq \alpha Q^{l}, \\
\left(f_{1}\left(\bar{\rho}_{1}^{l}\right), f_{2}\left(\bar{\rho}_{2}^{l}\right), f_{3}\left(\bar{\rho}_{3}^{l}\right)\right)=\left(\alpha Q^{l},(1-\alpha) Q^{l}, Q^{l}\right) & \text { if } Q^{l}-\Delta_{2,0}<\alpha Q^{l}<\Delta_{1,0}, \\
\left(f_{1}\left(\bar{\rho}_{1}^{l}\right), f_{2}\left(\bar{\rho}_{2}^{l}\right), f_{3}\left(\bar{\rho}_{3}^{l}\right)\right)=\left(Q^{l}-\Delta_{2,0}, \Delta_{2,0}, Q^{l}\right) & \text { if } \alpha Q^{l} \leq Q^{l}-\Delta_{2,0}<\Delta_{1,0},
\end{array}
$$

where $Q^{l}=Q^{l}\left(\vec{\rho}_{0}\right)$ and $\Delta_{i, 0}=\Delta_{i}\left(\rho_{i, 0}\right), i \in \mathrm{I}$. We start with a case by case analysis. Below we omit the superscript "HBC" and write $Q$ in place of $Q^{\mathrm{HBC}}$ and so on. We also let $\left(\tau_{1}^{k}, \tau_{2}^{k}, \tau_{3}^{k}\right)=\mathcal{T}^{k}\left[\vec{\rho}_{0}\right]$ for $k \geq 2$, $\Sigma_{3,0}=\Sigma_{3}\left(\rho_{3,0}\right)$ and $f_{h, 0}=f_{h}\left(\rho_{h, 0}\right)$ for $h \in \mathbf{H}$.

(GGG) If $\vec{\rho}_{0} \in \mathrm{GGG}$, then $\Delta_{i, 0}=f_{i}^{\max }, i \in \mathrm{I}, \Sigma_{3,0}=f_{3}^{\max }$ and therefore

$$
Q\left(\vec{\rho}_{0}\right)=g_{\min }<f_{3}^{\max }<f_{1}^{\max }+f_{2}^{\max } .
$$

As a consequence

$$
\Gamma_{1}\left(\vec{\rho}_{0}\right)=\left\{\begin{array}{ll}
f_{1}^{\max } & \text { if } \alpha g_{\min } \geq f_{1}^{\max }, \\
\alpha g_{\min } & \text { if } g_{\min }-f_{2}^{\max }<\alpha g_{\min }<f_{1}^{\max }, \\
g_{\min }-f_{2}^{\max } & \text { if } \alpha g_{\min } \leq g_{\min }-f_{2}^{\max },
\end{array} \quad \Gamma_{2}\left(\vec{\rho}_{0}\right)=g_{\min }-\Gamma_{1}\left(\vec{\rho}_{0}\right),\right.
$$

and therefore

$$
\mathcal{T}\left[\vec{\rho}_{0}\right]= \begin{cases}\left(\rho_{1, c}, \hat{\rho}_{2}\left(g_{\min }-f_{1}^{\max }\right), \check{\rho}_{3}\left(g_{\min }\right)\right) \in \mathrm{GGG} & \text { if } \alpha g_{\min } \geq f_{1}^{\max } \\ \left(\hat{\rho}_{1}\left(\alpha g_{\min }\right), \hat{\rho}_{2}\left((1-\alpha) g_{\min }\right), \check{\rho}_{3}\left(g_{\min }\right)\right) \in \mathrm{GGG} & \text { if } g_{\min }-f_{2}^{\max }<\alpha g_{\min }<f_{1}^{\max } \\ \left(\hat{\rho}_{1}\left(g_{\min }-f_{2}^{\max }\right), \rho_{2, c}, \check{\rho}_{3}\left(g_{\min }\right)\right) \in \mathrm{GGG} & \text { if } \alpha g_{\min } \leq g_{\min }-f_{2}^{\max }\end{cases}
$$

Notice that $\mathcal{T}\left[\vec{\rho}_{0}\right]$ depends on the geometry of the problem and not on the actual value of $\vec{\rho}_{0}$. By applying the above procedure to $\mathcal{T}\left[\vec{\rho}_{0}\right] \in \mathrm{GGG}$, we obtain that it is a fixed point for $\mathcal{T}$. Thus we have

$$
\mathcal{T}^{k}\left[\vec{\rho}_{0}\right]= \begin{cases}\left(\rho_{1, c}, \hat{\rho}_{2}\left(g_{\min }-f_{1}^{\max }\right), \check{\rho}_{3}\left(g_{\min }\right)\right) \in \mathrm{GGG} & \text { if } \alpha g_{\min } \geq f_{1}^{\max }, \\ \left(\hat{\rho}_{1}\left(\alpha g_{\min }\right), \hat{\rho}_{2}\left((1-\alpha) g_{\min }\right), \check{\rho}_{3}\left(g_{\min }\right)\right) \in \mathrm{GGG} & \text { if } g_{\min }-f_{2}^{\max }<\alpha g_{\min }<f_{1}^{\max }, \quad k \geq 1 \\ \left(\hat{\rho}_{1}\left(g_{\min }-f_{2}^{\max }\right), \rho_{2, c}, \check{\rho}_{3}\left(g_{\min }\right)\right) \in \mathrm{GGG} & \text { if } \alpha g_{\min } \leq g_{\min }-f_{2}^{\max },\end{cases}
$$

Since $Q\left(\vec{\rho}_{0}\right)=Q\left(\mathcal{T}\left[\vec{\rho}_{0}\right]\right)=Q\left(\mathcal{T}^{2}\left[\vec{\rho}_{0}\right]\right)=g_{\text {min }}$, we have that $Q^{l}=Q\left(\vec{\rho}_{0}\right)$ and therefore $\vec{\rho}^{l}=\mathcal{R}_{j}\left[\vec{\rho}_{0}\right]$. Hence $(6.8)$ holds because $\bar{\rho}_{h}^{l}=\tau_{h}=\tau_{h}^{3}, h \in \mathrm{H}$.

(BGG) If $\vec{\rho}_{0} \in \mathrm{BGG}$, then $\Delta_{1,0}=f_{1,0}, \Delta_{2,0}=f_{2}^{\max }$ and $\Sigma_{3,0}=f_{3}^{\max }$. We distinguish the following cases: (BGG.i) If $f_{1,0}+f_{2}^{\max }>f_{3}^{\max }$, then

$$
g_{\min } \leq g\left(f_{1,0}+f_{2}^{\max }\right)=Q\left(\vec{\rho}_{0}\right)<f_{3}^{\max }<f_{1,0}+f_{2}^{\max } .
$$

As a consequence

$$
\Gamma_{1}\left(\vec{\rho}_{0}\right)=\left\{\begin{array}{ll}
f_{1,0} & \text { if } \alpha Q\left(\vec{\rho}_{0}\right) \geq f_{1,0}, \\
\alpha Q\left(\vec{\rho}_{0}\right) & \text { if } Q\left(\vec{\rho}_{0}\right)-f_{2}^{\max }<\alpha Q\left(\vec{\rho}_{0}\right)<f_{1,0}, \\
Q\left(\vec{\rho}_{0}\right)-f_{2}^{\max } & \text { if } \alpha Q\left(\vec{\rho}_{0}\right) \leq Q\left(\vec{\rho}_{0}\right)-f_{2}^{\max },
\end{array} \quad \Gamma_{2}\left(\vec{\rho}_{0}\right)=Q\left(\vec{\rho}_{0}\right)-\Gamma_{1}\left(\vec{\rho}_{0}\right),\right.
$$


and therefore

$$
\mathcal{T}\left[\vec{\rho}_{0}\right]= \begin{cases}\left(\rho_{1,0}, \hat{\rho}_{2}\left(Q\left(\vec{\rho}_{0}\right)-f_{1,0}\right), \check{\rho}_{3}\left(Q\left(\vec{\rho}_{0}\right)\right)\right) \in \mathrm{BGG} & \text { if } \alpha Q\left(\vec{\rho}_{0}\right) \geq f_{1,0}, \\ \left(\hat{\rho}_{1}\left(\alpha Q\left(\vec{\rho}_{0}\right)\right), \hat{\rho}_{2}\left((1-\alpha) Q\left(\vec{\rho}_{0}\right)\right), \check{\rho}_{3}\left(Q\left(\vec{\rho}_{0}\right)\right)\right) \in \mathrm{GGG} & \text { if } Q\left(\vec{\rho}_{0}\right)-f_{2}^{\max }<\alpha Q\left(\vec{\rho}_{0}\right)<f_{1,0} \\ \left(\hat{\rho}_{1}\left(Q\left(\vec{\rho}_{0}\right)-f_{2}^{\max }\right), \rho_{2, c}, \check{\rho}_{3}\left(Q\left(\vec{\rho}_{0}\right)\right)\right) \in \mathrm{GGG} & \text { if } \alpha Q\left(\vec{\rho}_{0}\right) \leq Q\left(\vec{\rho}_{0}\right)-f_{2}^{\max }\end{cases}
$$

Notice that $\mathcal{T}\left[\vec{\rho}_{0}\right]$ depends on the geometry of the problem and $\rho_{1,0}$ alone.

(BGG.i-1) If $\alpha Q\left(\vec{\rho}_{0}\right) \geq f_{1,0}$, then we can apply the above procedure to $\mathcal{T}\left[\vec{\rho}_{0}\right] \in$ BGG and obtain that it is a fixed point for $\mathcal{T}$ because $Q\left(\mathcal{T}\left[\vec{\rho}_{0}\right]\right)=Q\left(\vec{\rho}_{0}\right)$. Thus we have

$$
\mathcal{T}^{k}\left[\vec{\rho}_{0}\right]=\left(\rho_{1,0}, \hat{\rho}_{2}\left(Q\left(\vec{\rho}_{0}\right)-f_{1,0}\right), \check{\rho}_{3}\left(Q\left(\vec{\rho}_{0}\right)\right)\right) \in \mathrm{BGG}, \quad k \geq 1 .
$$

Since $Q\left(\vec{\rho}_{0}\right)=Q\left(\mathcal{T}\left[\vec{\rho}_{0}\right]\right)=Q\left(\mathcal{T}^{2}\left[\vec{\rho}_{0}\right]\right)$, we have that $Q^{l}=Q\left(\vec{\rho}_{0}\right)$ and therefore $\vec{\rho}^{l}=\mathcal{R}_{j}\left[\vec{\rho}_{0}\right]$. Hence (6.8) holds because $\bar{\rho}_{h}^{l}=\tau_{h}=\tau_{h}^{3}, h \in \mathrm{H}$.

(BGG.i-2) If $Q\left(\vec{\rho}_{0}\right)-f_{2}^{\max }<\alpha Q\left(\vec{\rho}_{0}\right)<f_{1,0}$, then $g_{\min }-f_{2}^{\max }<\alpha g_{\min }<f_{1,0}<f_{1}^{\max }$ and we can apply the procedure used in $(\mathrm{GGG})$ to $\mathcal{T}\left[\vec{\rho}_{0}\right] \in \mathrm{GGG}$ and obtain that

$$
\mathcal{T}^{k}\left[\vec{\rho}_{0}\right]=\left(\hat{\rho}_{1}\left(\alpha g_{\min }\right), \hat{\rho}_{2}\left((1-\alpha) g_{\min }\right), \check{\rho}_{3}\left(g_{\min }\right)\right) \in \mathrm{GGG}, \quad k \geq 2 .
$$

Since $Q\left(\vec{\rho}_{0}\right)=g\left(f_{1,0}+f_{2}^{\max }\right) \geq g_{\min }=Q\left(\mathcal{T}\left[\vec{\rho}_{0}\right]\right)=Q\left(\mathcal{T}^{2}\left[\vec{\rho}_{0}\right]\right)$, we have that $Q^{l}=g_{\min }$. Since $Q^{l}-\Delta_{2,0}<$ $\alpha Q^{l}<\Delta_{1,0}$, by $(6.9 \mathrm{c})$ we have

$$
f_{1}\left(\bar{\rho}_{1}^{l}\right)=\alpha g_{\min }, \quad f_{2}\left(\bar{\rho}_{2}^{l}\right)=(1-\alpha) g_{\min }, \quad f_{3}\left(\bar{\rho}_{3}^{l}\right)=g_{\min } .
$$

Hence (6.8) holds because $\bar{\rho}_{h}^{l}=\tau_{h}^{2}=\tau_{h}^{3}, h \in \mathrm{H}$.

(BGG.i-3) If $(1-\alpha) g_{\min } \leq f_{2}^{\max } \leq(1-\alpha) Q\left(\vec{\rho}_{0}\right)$, then $\alpha g_{\min } \leq \alpha Q\left(\vec{\rho}_{0}\right) \leq Q\left(\vec{\rho}_{0}\right)-f_{2}^{\max } \leq f_{3}^{\max }-f_{2}^{\max }<$ $f_{1,0}<f_{1}^{\max }$ and by applying the procedure used in (GGG) to $\mathcal{T}\left[\vec{\rho}_{0}\right] \in \mathrm{GGG}$ we obtain that

$$
\mathcal{T}^{k}\left[\vec{\rho}_{0}\right]=\left(\hat{\rho}_{1}\left(\alpha g_{\text {min }}\right), \hat{\rho}_{2}\left((1-\alpha) g_{\text {min }}\right), \check{\rho}_{3}\left(g_{\text {min }}\right)\right) \in \text { GGG, } \quad k \geq 2 .
$$

Since $Q\left(\vec{\rho}_{0}\right)=g\left(f_{1,0}+f_{2}^{\max }\right) \geq g_{\min }=Q\left(\mathcal{T}\left[\vec{\rho}_{0}\right]\right)=Q\left(\mathcal{T}^{2}\left[\vec{\rho}_{0}\right]\right)$, we have that $Q^{l}=g_{\text {min }}$. Since $Q^{l}-\Delta_{2,0}<$ $\alpha Q^{l}<\Delta_{1,0}$, by $(6.9 \mathrm{c})$ we have

$$
f_{1}\left(\bar{\rho}_{1}^{l}\right)=\alpha g_{\min }, \quad f_{2}\left(\bar{\rho}_{2}^{l}\right)=(1-\alpha) g_{\min }, \quad f_{3}\left(\bar{\rho}_{3}^{l}\right)=g_{\min } .
$$

Hence (6.8) holds because $\bar{\rho}_{h}^{l}=\tau_{h}^{2}=\tau_{h}^{3}, h \in \mathrm{H}$.

(BGG.i-4) If $f_{2}^{\max } \leq(1-\alpha) g_{\min } \leq(1-\alpha) Q\left(\vec{\rho}_{0}\right)$, then we can apply the procedure used in (GGG) to $\mathcal{T}\left[\vec{\rho}_{0}\right] \in \mathrm{GGG}$ and obtain that

$$
\mathcal{T}^{k}\left[\vec{\rho}_{0}\right]=\left(\hat{\rho}_{1}\left(g_{\min }-f_{2}^{\max }\right), \rho_{2, c}, \check{\rho}_{3}\left(g_{\min }\right)\right) \in \mathrm{GGG}, \quad k \geq 2 .
$$

Since $Q\left(\vec{\rho}_{0}\right)=g\left(f_{1,0}+f_{2}^{\max }\right) \geq g_{\min }=Q\left(\mathcal{T}\left[\vec{\rho}_{0}\right]\right)=Q\left(\mathcal{T}^{2}\left[\vec{\rho}_{0}\right]\right)$, we have that $Q^{l}=g_{\min }$. Since $\alpha Q^{l} \leq Q^{l}-\Delta_{2,0}<$ $f_{3}^{\max }-\Delta_{2,0}<\Delta_{1,0}$, by (6.9d) we have

$$
f_{1}\left(\bar{\rho}_{1}^{l}\right)=g_{\min }-f_{2}^{\max }, \quad f_{2}\left(\bar{\rho}_{2}^{l}\right)=f_{2}^{\max }, \quad f_{3}\left(\bar{\rho}_{3}^{l}\right)=g_{\min } .
$$

Hence (6.8) holds because $\bar{\rho}_{h}^{l}=\tau_{h}^{2}=\tau_{h}^{3}, h \in \mathrm{H}$.

(BGG.ii) If $f_{1,0}+f_{2}^{\max } \leq f_{3}^{\max }$, then

$$
Q\left(\vec{\rho}_{0}\right)=\min \left\{f_{3}^{\max }, g\left(f_{1,0}+f_{2}^{\max }\right)\right\}=f_{3}^{\max } \geq f_{1,0}+f_{2}^{\max } .
$$

As a consequence $\Gamma_{1}\left(\vec{\rho}_{0}\right)=f_{1,0}, \Gamma_{2}\left(\vec{\rho}_{0}\right)=f_{2}^{\max }$, and therefore

$$
\mathcal{T}\left[\vec{\rho}_{0}\right]=\left(\rho_{1,0}, \rho_{2, c}, \check{\rho}_{3}\left(f_{1,0}+f_{2}^{\max }\right)\right) \in \text { BGG. }
$$


Notice that $\mathcal{T}\left[\vec{\rho}_{0}\right]$ depends on the geometry of the problem and $\rho_{1,0}$ alone. By applying the above procedure to $\mathcal{T}\left[\vec{\rho}_{0}\right] \in$ BGG, we obtain that it is a fixed point for $\mathcal{T}$ because $Q\left(\mathcal{T}\left[\vec{\rho}_{0}\right]\right)=Q\left(\vec{\rho}_{0}\right)$. Thus we have

$$
\mathcal{T}^{k}\left[\vec{\rho}_{0}\right]=\left(\rho_{1,0}, \rho_{2, c}, \check{\rho}_{3}\left(f_{1,0}+f_{2}^{\max }\right)\right) \in \mathrm{BGG}, \quad k \geq 1 .
$$

Since $Q\left(\vec{\rho}_{0}\right)=Q\left(\mathcal{T}\left[\vec{\rho}_{0}\right]\right)=Q\left(\mathcal{T}^{2}\left[\vec{\rho}_{0}\right]\right)$, we have that $Q^{l}=Q\left(\vec{\rho}_{0}\right)$ and therefore $\vec{\rho}^{l}=\mathcal{R}_{j}\left[\vec{\rho}_{0}\right]$. Hence (6.8) holds because $\bar{\rho}_{h}^{l}=\tau_{h}=\tau_{h}^{3}, h \in \mathrm{H}$. Notice that this case corresponds to (6.9a).

(GBG) The case $\vec{\rho}_{0} \in \mathrm{GBG}$ is analogous to the case (BGG).

(GGB) If $\vec{\rho}_{0} \in \mathrm{GGB}$, then $\Delta_{i, 0}=f_{i}^{\max }, i \in \mathrm{I}$, and $\Sigma_{3,0}=f_{3,0}$. Moreover

$$
Q\left(\vec{\rho}_{0}\right)=\min \left\{f_{3,0}, g_{\min }\right\} \leq g_{\min }<f_{3}^{\max }<f_{1}^{\max }+f_{2}^{\max }
$$

and therefore

$$
\Gamma_{1}\left(\vec{\rho}_{0}\right)=\left\{\begin{array}{ll}
f_{1}^{\max } & \text { if } \alpha Q\left(\vec{\rho}_{0}\right) \geq f_{1}^{\max }, \\
\alpha Q\left(\vec{\rho}_{0}\right) & \text { if } Q\left(\vec{\rho}_{0}\right)-f_{2}^{\max }<\alpha Q\left(\vec{\rho}_{0}\right)<f_{1}^{\max }, \\
Q\left(\vec{\rho}_{0}\right)-f_{2}^{\max } & \text { if } \alpha Q\left(\vec{\rho}_{0}\right) \leq Q\left(\vec{\rho}_{0}\right)-f_{2}^{\max },
\end{array} \quad \Gamma_{2}\left(\vec{\rho}_{0}\right)=Q\left(\vec{\rho}_{0}\right)-\Gamma_{1}\left(\vec{\rho}_{0}\right) .\right.
$$

(GGB.i) If $f_{3,0} \leq g_{\min }$, then $Q\left(\vec{\rho}_{0}\right)=f_{3,0}$ and

$$
\mathcal{T}\left[\vec{\rho}_{0}\right]= \begin{cases}\left(\rho_{1, c}, \hat{\rho}_{2}\left(f_{3,0}-f_{1}^{\max }\right), \rho_{3,0}\right) \in \mathrm{GGB} & \text { if } \alpha f_{3,0} \geq f_{1}^{\max } \\ \left(\hat{\rho}_{1}\left(\alpha f_{3,0}\right), \hat{\rho}_{2}\left((1-\alpha) f_{3,0}\right), \rho_{3,0}\right) \in \mathrm{GGB} & \text { if } f_{3,0}-f_{2}^{\max }<\alpha f_{3,0}<f_{1}^{\max }, \\ \left(\hat{\rho}_{1}\left(f_{3,0}-f_{2}^{\max }\right), \rho_{2, c}, \rho_{3,0}\right) \in \mathrm{GGB} & \text { if } \alpha f_{3,0} \leq f_{3,0}-f_{2}^{\max }\end{cases}
$$

We can apply the above procedure to $\mathcal{T}\left[\vec{\rho}_{0}\right] \in$ GGB obtaining that it is a fixed point for $\mathcal{T}$ because $Q\left(\mathcal{T}\left[\vec{\rho}_{0}\right]\right)=$ $Q\left(\vec{\rho}_{0}\right)$ and $\mathcal{T}\left[\vec{\rho}_{0}\right]$ depends on the geometry of the problem and $\rho_{3,0}$ alone. Thus we have

$$
\mathcal{T}^{k}\left[\vec{\rho}_{0}\right]=\left\{\begin{array}{ll}
\left(\rho_{1, c}, \hat{\rho}_{2}\left(f_{3,0}-f_{1}^{\max }\right), \rho_{3,0}\right) \in \mathrm{GGB} & \text { if } \alpha f_{3,0} \geq f_{1}^{\max }, \\
\left(\hat{\rho}_{1}\left(\alpha f_{3,0}\right), \hat{\rho}_{2}\left((1-\alpha) f_{3,0}\right), \rho_{3,0}\right) \in \mathrm{GGB} & \text { if } f_{3,0}-f_{2}^{\max }<\alpha f_{3,0}<f_{1}^{\max }, \\
\left(\hat{\rho}_{1}\left(f_{3,0}-f_{2}^{\max }\right), \rho_{2, c}, \rho_{3,0}\right) \in \mathrm{GGB} & \text { if } \alpha f_{3,0} \leq f_{3,0}-f_{2}^{\max },
\end{array} \quad k \geq 1 .\right.
$$

Since $Q\left(\vec{\rho}_{0}\right)=Q\left(\mathcal{T}\left[\vec{\rho}_{0}\right]\right)=Q\left(\mathcal{T}^{2}\left[\vec{\rho}_{0}\right]\right)$, we have that $Q^{l}=Q\left(\vec{\rho}_{0}\right)$ and therefore $\vec{\rho}^{l}=\mathcal{R}_{j}\left[\vec{\rho}_{0}\right]$. Hence (6.8) holds because $\bar{\rho}_{h}^{l}=\tau_{h}=\tau_{h}^{3}, h \in \mathrm{H}$.

(GGB.ii) If $f_{3,0}>g_{\min }$, then $Q\left(\vec{\rho}_{0}\right)=g_{\min }$ and $\mathcal{T}\left[\vec{\rho}_{0}\right]$ is given by $(6.10)$, which is a fixed point for $\mathcal{T}$. Thus we have

$$
\mathcal{T}^{k}\left[\vec{\rho}_{0}\right]= \begin{cases}\left(\rho_{1, c}, \hat{\rho}_{2}\left(g_{\min }-f_{1}^{\max }\right), \check{\rho}_{3}\left(g_{\min }\right)\right) \in \mathrm{GGG} & \text { if } \alpha g_{\min } \geq f_{1}^{\max }, \\ \left(\hat{\rho}_{1}\left(\alpha g_{\min }\right), \hat{\rho}_{2}\left((1-\alpha) g_{\min }\right), \check{\rho}_{3}\left(g_{\min }\right)\right) \in \mathrm{GGG} & \text { if } g_{\min }-f_{2}^{\max }<\alpha g_{\min }<f_{1}^{\max }, \quad k \geq 1 \\ \left(\hat{\rho}_{1}\left(g_{\min }-f_{2}^{\max }\right), \rho_{2, c}, \check{\rho}_{3}\left(g_{\min }\right)\right) \in \mathrm{GGG} & \text { if } \alpha g_{\min } \leq g_{\min }-f_{2}^{\max }\end{cases}
$$

Since $Q\left(\vec{\rho}_{0}\right)=Q\left(\mathcal{T}\left[\vec{\rho}_{0}\right]\right)=Q\left(\mathcal{T}^{2}\left[\vec{\rho}_{0}\right]\right)$, we have that $Q^{l}=Q\left(\vec{\rho}_{0}\right)$ and therefore $\vec{\rho}^{l}=\mathcal{R}_{j}\left[\vec{\rho}_{0}\right]$. Hence (6.8) holds because $\bar{\rho}_{h}^{l}=\tau_{h}=\tau_{h}^{3}, h \in \mathrm{H}$.

(BBG) If $\vec{\rho}_{0} \in \mathrm{BBG}$, then $\Delta_{i, 0}=f_{i, 0}, i \in \mathrm{I}, \Sigma_{3,0}=f_{3}^{\max }$ and therefore

$$
Q\left(\vec{\rho}_{0}\right)=\min \left\{f_{3}^{\max }, g\left(f_{1,0}+f_{2,0}\right)\right\}=g\left(f_{1,0}+f_{2,0}\right) \geq g_{\min } .
$$

We distinguish the following cases:

(BBG.i) If $f_{1,0}+f_{2,0} \leq Q\left(\vec{\rho}_{0}\right)$, then by Remark 2.10 we have $Q\left(\vec{\rho}_{0}\right)=f_{3}^{\max }$. Moreover $\Gamma_{i}\left(\vec{\rho}_{0}\right)=f_{i, 0}, i \in \mathrm{I}$, and therefore

$$
\mathcal{T}\left[\vec{\rho}_{0}\right]=\left(\rho_{1,0}, \rho_{2,0}, \check{\rho}_{3}\left(f_{1,0}+f_{2,0}\right)\right) \in \mathrm{BBG}
$$


Notice that $\mathcal{T}\left[\vec{\rho}_{0}\right]$ depends on the geometry of the problem and $\left(\rho_{1,0}, \rho_{2,0}\right)$ alone. By applying the above procedure to $\mathcal{T}\left[\vec{\rho}_{0}\right] \in$ BBG, we obtain that it is a fixed point for $\mathcal{T}$ because $Q\left(\mathcal{T}\left[\vec{\rho}_{0}\right]\right)=Q\left(\vec{\rho}_{0}\right)$. Thus we have

$$
\mathcal{T}^{k}\left[\vec{\rho}_{0}\right]=\left(\rho_{1,0}, \rho_{2,0}, \check{\rho}_{3}\left(f_{1,0}+f_{2,0}\right)\right) \in \mathrm{BBG}, \quad k \geq 1 .
$$

Since $Q\left(\vec{\rho}_{0}\right)=Q\left(\mathcal{T}\left[\vec{\rho}_{0}\right]\right)=Q\left(\mathcal{T}^{2}\left[\vec{\rho}_{0}\right]\right)$, we have that $Q^{l}=Q\left(\vec{\rho}_{0}\right)$ and therefore $\vec{\rho}^{l}=\mathcal{R}_{j}\left[\vec{\rho}_{0}\right]$. Hence (6.8) holds because $\bar{\rho}_{h}^{l}=\tau_{h}=\tau_{h}^{3}, h \in \mathrm{H}$. Notice that this case corresponds to (6.9a).

(BBG.ii) If $f_{1,0}+f_{2,0}>Q\left(\vec{\rho}_{0}\right)$, then

$$
\Gamma_{1}\left(\vec{\rho}_{0}\right)=\left\{\begin{array}{ll}
f_{1,0} & \text { if } \alpha Q\left(\vec{\rho}_{0}\right) \geq f_{1,0}, \\
\alpha Q\left(\vec{\rho}_{0}\right) & \text { if } Q\left(\vec{\rho}_{0}\right)-f_{2,0}<\alpha Q\left(\vec{\rho}_{0}\right)<f_{1,0}, \\
Q\left(\vec{\rho}_{0}\right)-f_{2,0} & \text { if } \alpha Q\left(\vec{\rho}_{0}\right) \leq Q\left(\vec{\rho}_{0}\right)-f_{2,0},
\end{array} \quad \Gamma_{2}\left(\vec{\rho}_{0}\right)=Q\left(\vec{\rho}_{0}\right)-\Gamma_{1}\left(\vec{\rho}_{0}\right) ;\right.
$$

moreover

$$
\mathcal{T}\left[\vec{\rho}_{0}\right]= \begin{cases}\left(\rho_{1,0}, \hat{\rho}_{2}\left(Q\left(\vec{\rho}_{0}\right)-f_{1,0}\right), \check{\rho}_{3}\left(Q\left(\vec{\rho}_{0}\right)\right)\right) \in \mathrm{BGG} & \text { if } \alpha Q\left(\vec{\rho}_{0}\right) \geq f_{1,0}, \\ \left(\hat{\rho}_{1}\left(\alpha Q\left(\vec{\rho}_{0}\right)\right), \hat{\rho}_{2}\left((1-\alpha) Q\left(\vec{\rho}_{0}\right)\right), \check{\rho}_{3}\left(Q\left(\vec{\rho}_{0}\right)\right)\right) \in \mathrm{GGG} & \text { if } Q\left(\vec{\rho}_{0}\right)-f_{2,0}<\alpha Q\left(\vec{\rho}_{0}\right)<f_{1,0} \\ \left(\hat{\rho}_{1}\left(Q\left(\vec{\rho}_{0}\right)-f_{2,0}\right), \rho_{2,0}, \check{\rho}_{3}\left(Q\left(\vec{\rho}_{0}\right)\right)\right) \in \mathrm{GBG} & \text { if } \alpha Q\left(\vec{\rho}_{0}\right) \leq Q\left(\vec{\rho}_{0}\right)-f_{2,0}\end{cases}
$$

Notice that $Q\left(\vec{\rho}_{0}\right) \geq Q\left(\mathcal{T}\left[\vec{\rho}_{0}\right]\right) \geq g_{\min }$.

(BBG.ii-1) If $\alpha g\left(f_{1,0}+f_{2}^{\max }\right) \geq f_{1,0}$, then $\alpha Q\left(\vec{\rho}_{0}\right) \geq f_{1,0}$ and by applying the procedure used in (BGG.i-1) to $\mathcal{T}\left[\vec{\rho}_{0}\right] \in \mathrm{BGG}$ we obtain that

$$
\mathcal{T}^{k}\left[\vec{\rho}_{0}\right]=\left(\rho_{1,0}, \hat{\rho}_{2}\left(Q\left(\mathcal{T}\left[\vec{\rho}_{0}\right]\right)-f_{1,0}\right), \check{\rho}_{3}\left(Q\left(\mathcal{T}\left[\vec{\rho}_{0}\right]\right)\right)\right) \in \mathrm{BGG}, \quad k \geq 2 .
$$

Since $Q\left(\vec{\rho}_{0}\right) \geq Q\left(\mathcal{T}\left[\vec{\rho}_{0}\right]\right)=Q\left(\mathcal{T}^{2}\left[\vec{\rho}_{0}\right]\right)=g\left(f_{1,0}+f_{2}^{\max }\right)$, we have that $Q^{l}=Q\left(\mathcal{T}\left[\vec{\rho}_{0}\right]\right)$. Since $Q^{l} \leq Q\left(\vec{\rho}_{0}\right)<$ $f_{1,0}+f_{2,0}=\Delta_{1,0}+\Delta_{2,0}$ and $\alpha Q^{l}=\alpha Q\left(\mathcal{T}\left[\vec{\rho}_{0}\right]\right) \geq f_{1,0}=\Delta_{1,0}$, we have $Q^{l}-\Delta_{2,0}<\Delta_{1,0} \leq \alpha Q^{l}$ and by $(6.9 \mathrm{~b})$

$$
f_{1}\left(\bar{\rho}_{1}^{l}\right)=f_{1,0}, \quad f_{2}\left(\bar{\rho}_{2}^{l}\right)=Q^{l}-f_{1,0}, \quad f_{3}\left(\bar{\rho}_{3}^{l}\right)=Q^{l} .
$$

Hence (6.8) holds because $\bar{\rho}_{h}^{l}=\tau_{h}^{2}=\tau_{h}^{3}, h \in \mathrm{H}$.

(BBG.ii-2) If $\alpha Q\left(\vec{\rho}_{0}\right) \geq f_{1,0}>\alpha g\left(f_{1,0}+f_{2}^{\max }\right)$, then $\alpha g_{\min } \leq \alpha g\left(f_{1,0}+f_{2}^{\max }\right)=\alpha Q\left(\mathcal{T}\left[\vec{\rho}_{0}\right]\right)<f_{1,0},(1-$ $\alpha) g_{\min } \leq(1-\alpha) Q\left(\mathcal{T}\left[\vec{\rho}_{0}\right]\right) \leq(1-\alpha) Q\left(\vec{\rho}_{0}\right) \leq Q\left(\vec{\rho}_{0}\right)-f_{1,0}<f_{2,0}<f_{2}^{\max }$ and by applying the procedure used in (BGG.i-2) to $\mathcal{T}\left[\vec{\rho}_{0}\right] \in$ BGG we obtain that

$$
\begin{array}{ll}
\mathcal{T}^{2}\left[\vec{\rho}_{0}\right] & =\left(\hat{\rho}_{1}\left(\alpha Q\left(\mathcal{T}\left[\vec{\rho}_{0}\right]\right)\right), \hat{\rho}_{2}\left((1-\alpha) Q\left(\mathcal{T}\left[\vec{\rho}_{0}\right]\right)\right), \check{\rho}_{3}\left(Q\left(\mathcal{T}\left[\vec{\rho}_{0}\right]\right)\right)\right) \in \mathrm{GGG}, \\
\mathcal{T}^{k}\left[\vec{\rho}_{0}\right]=\left(\hat{\rho}_{1}\left(\alpha g_{\min }\right), \hat{\rho}_{2}\left((1-\alpha) g_{\min }\right), \check{\rho}_{3}\left(g_{\min }\right)\right) \in \mathrm{GGG}, & k \geq 3
\end{array}
$$

Since $Q\left(\vec{\rho}_{0}\right) \geq Q\left(\mathcal{T}\left[\vec{\rho}_{0}\right]\right) \geq Q\left(\mathcal{T}^{2}\left[\vec{\rho}_{0}\right]\right)=g_{\min }$, we have that $Q^{l}=g_{\min }$. Since $(1-\alpha) Q^{l}<f_{2,0}=\Delta_{2,0}$ and $\alpha Q^{l}<f_{1,0}=\Delta_{1,0}$, we have $Q^{l}-\Delta_{2,0}<\alpha Q^{l}<\Delta_{1,0}$ and by $(6.9 \mathrm{c})$

$$
f_{1}\left(\bar{\rho}_{1}^{l}\right)=\alpha g_{\min }, \quad f_{2}\left(\bar{\rho}_{2}^{l}\right)=(1-\alpha) g_{\min }, \quad f_{3}\left(\bar{\rho}_{3}^{l}\right)=g_{\min } .
$$

Hence (6.8) holds because $\bar{\rho}_{h}^{l}=\tau_{h}^{3}, h \in \mathrm{H}$.

(BBG.ii-3) If $Q\left(\vec{\rho}_{0}\right)-f_{2,0}<\alpha Q\left(\vec{\rho}_{0}\right)<f_{1,0}$, then $g_{\min }-f_{2}^{\max }<g_{\min }-f_{2,0} \leq \alpha g_{\min }<f_{1,0}<f_{1}^{\max }$, hence we can apply the procedure used in (GGG) to $\mathcal{T}\left[\vec{\rho}_{0}\right] \in$ GGG and obtain that

$$
\mathcal{T}^{k}\left[\vec{\rho}_{0}\right]=\left(\hat{\rho}_{1}\left(\alpha g_{\min }\right), \hat{\rho}_{2}\left((1-\alpha) g_{\min }\right), \check{\rho}_{3}\left(g_{\min }\right)\right) \in \mathrm{GGG}, \quad k \geq 2 .
$$

Since $Q\left(\vec{\rho}_{0}\right) \geq g_{\min }=Q\left(\mathcal{T}\left[\vec{\rho}_{0}\right]\right)=Q\left(\mathcal{T}^{2}\left[\vec{\rho}_{0}\right]\right)$, we have that $Q^{l}=g_{\min }$. Since $Q^{l}-\Delta_{2,0}<\alpha Q^{l}<\Delta_{1,0}$, by $(6.9 \mathrm{c})$

$$
f_{1}\left(\bar{\rho}_{1}^{l}\right)=\alpha g_{\min } \quad f_{2}\left(\bar{\rho}_{2}^{l}\right)=(1-\alpha) g_{\min }, \quad f_{3}\left(\bar{\rho}_{3}^{l}\right)=g_{\min } .
$$


Hence (6.8) holds because $\bar{\rho}_{h}^{l}=\tau_{h}^{2}=\tau_{h}^{3}, h \in \mathrm{H}$.

(BBG.ii-4) If $\alpha Q\left(\vec{\rho}_{0}\right) \leq Q\left(\vec{\rho}_{0}\right)-f_{2,0}$, then we can proceed as in (BBG.ii-1), and we call this case (BBG.ii-4.a), or as in (BBG.ii-2), and we call such case (BBG.ii-4.b).

(BGB) If $\vec{\rho}_{0} \in \mathrm{BGB}$, then $\Delta_{1,0}=f_{1,0}, \Delta_{2,0}=f_{2}^{\max }$ and $\Sigma_{3,0}=f_{3,0}$. Clearly

$$
Q\left(\vec{\rho}_{0}\right)=\min \left\{f_{3,0}, g\left(f_{1,0}+f_{2}^{\max }\right)\right\} \leq g\left(f_{1,0}+f_{2}^{\max }\right) .
$$

We distinguish the following cases:

(BGB.i) If $f_{3,0}=f_{1,0}+f_{2}^{\max } \leq Q\left(\vec{\rho}_{0}\right)$, then by Remark 2.10 we have that $g\left(f_{1,0}+f_{2}^{\max }\right)=f_{3}^{\max }$ and $Q\left(\vec{\rho}_{0}\right)=f_{3,0}$. Moreover $\Gamma_{1}\left(\vec{\rho}_{0}\right)=f_{1,0}, \Gamma_{2}\left(\vec{\rho}_{0}\right)=f_{2}^{\max }$, and therefore

$$
\mathcal{T}\left[\vec{\rho}_{0}\right]=\left(\rho_{1,0}, \rho_{2, c}, \rho_{3,0}\right) \in \mathrm{BGB} .
$$

Notice that $\mathcal{T}\left[\vec{\rho}_{0}\right]$ depends on the geometry of the problem and $\left(\rho_{1,0}, \rho_{3,0}\right)$ alone. By applying the above procedure to $\mathcal{T}\left[\vec{\rho}_{0}\right] \in \mathrm{BGB}$, we obtain that it is a fixed point for $\mathcal{T}$ because $Q\left(\mathcal{T}\left[\vec{\rho}_{0}\right]\right)=Q\left(\vec{\rho}_{0}\right)$. Thus we have

$$
\mathcal{T}^{k}\left[\vec{\rho}_{0}\right]=\left(\rho_{1,0}, \rho_{2, c}, \rho_{3,0}\right) \in \mathrm{BGB}, \quad k \geq 1 .
$$

Since $Q\left(\vec{\rho}_{0}\right)=Q\left(\mathcal{T}\left[\vec{\rho}_{0}\right]\right)=Q\left(\mathcal{T}^{2}\left[\vec{\rho}_{0}\right]\right)$, we have that $Q^{l}=Q\left(\vec{\rho}_{0}\right)$ and therefore $\vec{\rho}^{l}=\mathcal{R}_{j}\left[\vec{\rho}_{0}\right]$. Hence (6.8) holds because $\bar{\rho}_{h}^{l}=\tau_{h}=\tau_{h}^{3}, h \in \mathrm{H}$. Notice that this case corresponds to (6.9a).

(BGB.ii) If $f_{3,0} \neq f_{1,0}+f_{2}^{\max } \leq Q\left(\vec{\rho}_{0}\right)$, then by Remark 2.10 we have $g\left(f_{1,0}+f_{2}^{\max }\right)=f_{3}^{\max }$ and $Q\left(\vec{\rho}_{0}\right)=f_{3,0}$. Moreover $\Gamma_{1}\left(\vec{\rho}_{0}\right)=f_{1,0}, \Gamma_{2}\left(\vec{\rho}_{0}\right)=f_{2}^{\max }$, and therefore

$$
\mathcal{T}\left[\vec{\rho}_{0}\right]=\left(\rho_{1,0}, \rho_{2, c}, \check{\rho}_{3}\left(f_{1,0}+f_{2}^{\max }\right)\right) \in \text { BGG. }
$$

Notice that $f_{3}^{\max }=Q\left(\mathcal{T}\left[\vec{\rho}_{0}\right]\right) \geq Q\left(\vec{\rho}_{0}\right)=f_{3,0} \geq f_{1,0}+f_{2}^{\max }$. By applying the procedure used in (BGG.ii) to $\mathcal{T}\left[\vec{\rho}_{0}\right] \in$ BGG, we obtain that it is a fixed point for $\mathcal{T}$ and therefore

$$
\mathcal{T}^{k}\left[\vec{\rho}_{0}\right]=\left(\rho_{1,0}, \rho_{2, c}, \check{\rho}_{3}\left(f_{1,0}+f_{2}^{\max }\right)\right) \in \mathrm{BGG}, \quad k \geq 1 .
$$

Since $Q\left(\vec{\rho}_{0}\right)=f_{3,0} \leq f_{3}^{\max }=Q\left(\mathcal{T}\left[\vec{\rho}_{0}\right]\right)=Q\left(\mathcal{T}^{2}\left[\vec{\rho}_{0}\right]\right)$, we have that $Q^{l}=Q\left(\vec{\rho}_{0}\right)$ and therefore $\vec{\rho}^{l}=\mathcal{R}_{j}\left[\vec{\rho}_{0}\right]$. Hence (6.8) holds because $\bar{\rho}_{h}^{l}=\tau_{h}=\tau_{h}^{3}, h \in \mathrm{H}$. Notice that this case corresponds to (6.9a).

(BGB.iii) If $f_{1,0}+f_{2}^{\max }>Q\left(\vec{\rho}_{0}\right) \neq f_{3,0}$, then

$$
\Gamma_{1}\left(\vec{\rho}_{0}\right)=\left\{\begin{array}{ll}
f_{1,0} & \text { if } \alpha Q\left(\vec{\rho}_{0}\right) \geq f_{1,0}, \\
\alpha Q\left(\vec{\rho}_{0}\right) & \text { if } Q\left(\vec{\rho}_{0}\right)-f_{2}^{\max }<\alpha Q\left(\vec{\rho}_{0}\right)<f_{1,0}, \\
Q\left(\vec{\rho}_{0}\right)-f_{2}^{\max } & \text { if } \alpha Q\left(\vec{\rho}_{0}\right) \leq Q\left(\vec{\rho}_{0}\right)-f_{2}^{\max },
\end{array} \quad \Gamma_{2}\left(\vec{\rho}_{0}\right)=Q\left(\vec{\rho}_{0}\right)-\Gamma_{1}\left(\vec{\rho}_{0}\right) ;\right.
$$

moreover

$$
\mathcal{T}\left[\vec{\rho}_{0}\right]= \begin{cases}\left(\rho_{1,0}, \hat{\rho}_{2}\left(Q\left(\vec{\rho}_{0}\right)-f_{1,0}\right), \check{\rho}_{3}\left(Q\left(\vec{\rho}_{0}\right)\right)\right) \in \mathrm{BGG} & \text { if } \alpha Q\left(\vec{\rho}_{0}\right) \geq f_{1,0}, \\ \left(\hat{\rho}_{1}\left(\alpha Q\left(\vec{\rho}_{0}\right)\right), \hat{\rho}_{2}\left((1-\alpha) Q\left(\vec{\rho}_{0}\right)\right), \check{\rho}_{3}\left(Q\left(\vec{\rho}_{0}\right)\right)\right) \in \mathrm{GGG} & \text { if } Q\left(\vec{\rho}_{0}\right)-f_{2}^{\max }<\alpha Q\left(\vec{\rho}_{0}\right)<f_{1,0} \\ \left(\hat{\rho}_{1}\left(Q\left(\vec{\rho}_{0}\right)-f_{2}^{\max }\right), \rho_{2, c}, \check{\rho}_{3}\left(Q\left(\vec{\rho}_{0}\right)\right)\right) \in \mathrm{GGG} & \text { if } \alpha Q\left(\vec{\rho}_{0}\right) \leq Q\left(\vec{\rho}_{0}\right)-f_{2}^{\max }\end{cases}
$$

Notice that $Q\left(\vec{\rho}_{0}\right)=g\left(f_{1,0}+f_{2}^{\max }\right)<f_{3,0}<f_{3}^{\max }$, whence $f_{1,0}+f_{2}^{\max }>f_{3}^{\max }$.

(BGB.iii-1) If $\alpha Q\left(\vec{\rho}_{0}\right) \geq f_{1,0}$, then $Q\left(\mathcal{T}\left[\vec{\rho}_{0}\right]\right)=Q\left(\vec{\rho}_{0}\right)$, hence we can apply the procedure used in (BGG.i-1) to $\mathcal{T}\left[\vec{\rho}_{0}\right] \in$ BGG and obtain that it is a fixed point for $\mathcal{T}$. Thus we have

$$
\mathcal{T}^{k}\left[\vec{\rho}_{0}\right]=\left(\rho_{1,0}, \hat{\rho}_{2}\left(Q\left(\vec{\rho}_{0}\right)-f_{1,0}\right), \check{\rho}_{3}\left(Q\left(\vec{\rho}_{0}\right)\right)\right) \in \mathrm{BGG}, \quad k \geq 1 .
$$

Since $Q\left(\vec{\rho}_{0}\right)=Q\left(\mathcal{T}\left[\vec{\rho}_{0}\right]\right)=Q\left(\mathcal{T}^{2}\left[\vec{\rho}_{0}\right]\right)$, we have that $Q^{l}=Q\left(\vec{\rho}_{0}\right)$ and therefore $\vec{\rho}^{l}=\mathcal{R}_{j}\left[\vec{\rho}_{0}\right]$. Hence (6.8) holds because $\bar{\rho}_{h}^{l}=\tau_{h}=\tau_{h}^{3}, h \in \mathrm{H}$. 
(BGB.iii-2) If $Q\left(\vec{\rho}_{0}\right)-f_{2}^{\max }<\alpha Q\left(\vec{\rho}_{0}\right)<f_{1,0}$, then $Q\left(\mathcal{T}\left[\vec{\rho}_{0}\right]\right)=g_{\min }$ and $g_{\min }-f_{2}^{\max }<\alpha g_{\min }<f_{1,0}<f_{1}^{\max }$, hence we can apply the procedure used in $(\mathrm{GGG})$ to $\mathcal{T}\left[\vec{\rho}_{0}\right] \in \mathrm{GGG}$ and obtain that

$$
\mathcal{T}^{k}\left[\vec{\rho}_{0}\right]=\left(\hat{\rho}_{1}\left(\alpha g_{\min }\right), \hat{\rho}_{2}\left((1-\alpha) g_{\min }\right), \check{\rho}_{3}\left(g_{\min }\right)\right) \in \mathrm{GGG}, \quad k \geq 2 .
$$

Since $Q\left(\vec{\rho}_{0}\right) \geq Q\left(\mathcal{T}\left[\vec{\rho}_{0}\right]\right)=Q\left(\mathcal{T}^{2}\left[\vec{\rho}_{0}\right]\right)=g_{\min }$, we have that $Q^{l}=g_{\min }$. Since $Q^{l}-\Delta_{2,0}<\alpha Q^{l}<\Delta_{1,0}$, by $(6.9 \mathrm{c})$

$$
f_{1}\left(\bar{\rho}_{1}^{l}\right)=\alpha g_{\min }, \quad f_{2}\left(\bar{\rho}_{2}^{l}\right)=(1-\alpha) g_{\min }, \quad f_{3}\left(\bar{\rho}_{3}^{l}\right)=g_{\min } .
$$

Hence (6.8) holds because $\bar{\rho}_{h}^{l}=\tau_{h}^{2}=\tau_{h}^{3}, h \in \mathrm{H}$.

(BGB.iii-3) If $\alpha Q\left(\vec{\rho}_{0}\right) \leq Q\left(\vec{\rho}_{0}\right)-f_{2}^{\max }$, then $Q\left(\mathcal{T}\left[\vec{\rho}_{0}\right]\right)=g_{\min }$ and $\alpha g_{\min } \leq \alpha Q\left(\vec{\rho}_{0}\right) \leq Q\left(\vec{\rho}_{0}\right)-f_{2}^{\max }<f_{1,0}<$ $f_{1}^{\max }$, hence we can apply the procedure used in (GGG) to $\mathcal{T}\left[\vec{\rho}_{0}\right] \in$ GGG obtaining that

$$
\mathcal{T}^{k}\left[\vec{\rho}_{0}\right]=\left\{\begin{array}{ll}
\left(\hat{\rho}_{1}\left(\alpha g_{\min }\right), \hat{\rho}_{2}\left((1-\alpha) g_{\min }\right), \check{\rho}_{3}\left(g_{\min }\right)\right) \in \mathrm{GGG} & \text { if } g_{\min }-f_{2}^{\max }<\alpha g_{\min }<f_{1}^{\max }, \\
\left(\hat{\rho}_{1}\left(g_{\min }-f_{2}^{\max }\right), \rho_{2, c}, \check{\rho}_{3}\left(g_{\min }\right)\right) \in \mathrm{GGG} & \text { if } \alpha g_{\min } \leq g_{\min }-f_{2}^{\max },
\end{array} \quad k \geq 2 .\right.
$$

Since $Q\left(\vec{\rho}_{0}\right) \geq Q\left(\mathcal{T}\left[\vec{\rho}_{0}\right]\right)=Q\left(\mathcal{T}^{2}\left[\vec{\rho}_{0}\right]\right)=g_{\text {min }}$, we have that $Q^{l}=g_{\text {min }}$. Clearly $\alpha Q^{l}<\Delta_{1,0}$ and $Q^{l} \leq Q\left(\vec{\rho}_{0}\right)<$ $f_{1,0}+f_{2}^{\max }=\Delta_{1,0}+\Delta_{2,0}$, by $(6.9 \mathrm{c})$ and $(6.9 \mathrm{~d})$ we have

$$
\begin{cases}f_{1}\left(\bar{\rho}_{1}^{l}\right)=\alpha g_{\min }, \quad f_{2}\left(\bar{\rho}_{2}^{l}\right)=(1-\alpha) g_{\min }, \quad f_{3}\left(\bar{\rho}_{3}^{l}\right)=g_{\min } & \text { if } g_{\min }-\Delta_{2,0}<\alpha g_{\min }<\Delta_{1,0}, \\ f_{1}\left(\bar{\rho}_{1}^{l}\right)=g_{\min }-\Delta_{2,0}, \quad f_{2}\left(\bar{\rho}_{2}^{l}\right)=\Delta_{2,0}, \quad f_{3}\left(\bar{\rho}_{3}^{l}\right)=g_{\min } & \text { if } \alpha g_{\min } \leq g_{\min }-\Delta_{2,0}<\Delta_{1,0} .\end{cases}
$$

Hence (6.8) holds because $\bar{\rho}_{h}^{l}=\tau_{h}^{2}=\tau_{h}^{3}, h \in \mathrm{H}$.

(BGB.iv) If $f_{1,0}+f_{2}^{\max }>Q\left(\vec{\rho}_{0}\right)=f_{3,0}$, then

$$
\Gamma_{1}\left(\vec{\rho}_{0}\right)=\left\{\begin{array}{ll}
f_{1,0} & \text { if } \alpha f_{3,0} \geq f_{1,0}, \\
\alpha f_{3,0} & \text { if } f_{3,0}-f_{2}^{\max }<\alpha f_{3,0}<f_{1,0}, \\
f_{3,0}-f_{2}^{\max } & \text { if } \alpha f_{3,0} \leq f_{3,0}-f_{2}^{\max },
\end{array} \quad \Gamma_{2}\left(\vec{\rho}_{0}\right)=f_{3,0}-\Gamma_{1}\left(\vec{\rho}_{0}\right) ;\right.
$$

moreover

$$
\mathcal{T}\left[\vec{\rho}_{0}\right]= \begin{cases}\left(\rho_{1,0}, \hat{\rho}_{2}\left(f_{3,0}-f_{1,0}\right), \rho_{3,0}\right) \in \mathrm{BGB} & \text { if } \alpha f_{3,0} \geq f_{1,0}, \\ \left(\hat{\rho}_{1}\left(\alpha f_{3,0}\right), \hat{\rho}_{2}\left((1-\alpha) f_{3,0}\right), \rho_{3,0}\right) \in \mathrm{GGB} & \text { if } f_{3,0}-f_{2}^{\max }<\alpha f_{3,0}<f_{1,0}, \\ \left(\hat{\rho}_{1}\left(f_{3,0}-f_{2}^{\max }\right), \rho_{2, c}, \rho_{3,0}\right) \in \mathrm{GGB} & \text { if } \alpha f_{3,0} \leq f_{3,0}-f_{2}^{\max } .\end{cases}
$$

Notice that $\mathcal{T}\left[\vec{\rho}_{0}\right]$ depends on the geometry of the problem and $\left(\rho_{1,0}, \rho_{3,0}\right)$ alone.

(BGB.iv-1) If $\alpha f_{3,0} \geq f_{1,0}$, then we can apply the above procedure to $\mathcal{T}\left[\vec{\rho}_{0}\right] \in$ BGB and obtain that it is a fixed point for $\mathcal{T}$ because $Q\left(\mathcal{T}\left[\vec{\rho}_{0}\right]\right)=Q\left(\vec{\rho}_{0}\right)=f_{3,0}$. Thus we have

$$
\mathcal{T}^{k}\left[\vec{\rho}_{0}\right]=\left(\rho_{1,0}, \hat{\rho}_{2}\left(f_{3,0}-f_{1,0}\right), \rho_{3,0}\right) \in \mathrm{BGB}, \quad k \geq 1 .
$$

Since $Q\left(\vec{\rho}_{0}\right)=f_{3,0}=Q\left(\mathcal{T}\left[\vec{\rho}_{0}\right]\right)=Q\left(\mathcal{T}^{2}\left[\vec{\rho}_{0}\right]\right)$, we have that $Q^{l}=Q\left(\vec{\rho}_{0}\right)$ and therefore $\vec{\rho}^{l}=\mathcal{R}_{j}\left[\vec{\rho}_{0}\right]$. Hence $(6.8)$ holds because $\bar{\rho}_{h}^{l}=\tau_{h}=\tau_{h}^{3}, h \in \mathrm{H}$.

(BGB.iv-2) If $f_{3,0}-f_{2}^{\max }<\alpha f_{3,0}<f_{1,0}$ and $f_{3,0} \leq g_{\min }$, then we can apply the procedure used in (GGB.i) to $\mathcal{T}\left[\vec{\rho}_{0}\right] \in \mathrm{GGB}$ and obtain that

$$
\mathcal{T}^{k}\left[\vec{\rho}_{0}\right]=\left(\hat{\rho}_{1}\left(\alpha f_{3,0}\right), \hat{\rho}_{2}\left((1-\alpha) f_{3,0}\right), \rho_{3,0}\right) \in \mathrm{GGB}, \quad k \geq 1 .
$$

Since $Q\left(\vec{\rho}_{0}\right)=f_{3,0}=Q\left(\mathcal{T}\left[\vec{\rho}_{0}\right]\right)=Q\left(\mathcal{T}^{2}\left[\vec{\rho}_{0}\right]\right)$, we have that $Q^{l}=Q\left(\vec{\rho}_{0}\right)$ and therefore $\vec{\rho}^{l}=\mathcal{R}_{j}\left[\vec{\rho}_{0}\right]$. Hence $(6.8)$ holds because $\bar{\rho}_{h}^{l}=\tau_{h}=\tau_{h}^{3}, h \in \mathrm{H}$.

(BGB.iv-3) If $f_{3,0}-f_{2}^{\max }<\alpha f_{3,0}<f_{1,0}$ and $f_{3,0}>g_{\min }$, then $g_{\min }-f_{2}^{\max }<\alpha g_{\min }<f_{1,0}<f_{1}^{\max }$, hence we can apply the procedure used in (GGB.ii) to $\mathcal{T}\left[\vec{\rho}_{0}\right] \in \mathrm{GGB}$, obtaining that $Q\left(\mathcal{T}\left[\vec{\rho}_{0}\right]\right)=g_{\text {min }}$ and

$$
\mathcal{T}^{k}\left[\vec{\rho}_{0}\right]=\left(\hat{\rho}_{1}\left(\alpha g_{\min }\right), \hat{\rho}_{2}\left((1-\alpha) g_{\min }\right), \check{\rho}_{3}\left(g_{\min }\right)\right) \in \mathrm{GGG}, \quad k \geq 2 .
$$


Since $Q\left(\vec{\rho}_{0}\right)=f_{3,0}>g_{\text {min }}=Q\left(\mathcal{T}\left[\vec{\rho}_{0}\right]\right)=Q\left(\mathcal{T}^{2}\left[\vec{\rho}_{0}\right]\right)$, we have that $Q^{l}=g_{\text {min }}$. Since $Q^{l}-\Delta_{2,0}<\alpha Q^{l}<\Delta_{1,0}$, by $(6.9 \mathrm{c})$

$$
f_{1}\left(\bar{\rho}_{1}^{l}\right)=\alpha g_{\min }, \quad f_{2}\left(\bar{\rho}_{2}^{l}\right)=(1-\alpha) g_{\min }, \quad f_{3}\left(\bar{\rho}_{3}^{l}\right)=g_{\min } .
$$

Hence (6.8) holds because $\bar{\rho}_{h}^{l}=\tau_{h}^{2}=\tau_{h}^{3}, h \in \mathrm{H}$.

(BGB.iv-4) If $\alpha f_{3,0} \leq f_{3,0}-f_{2}^{\max }$ and $f_{3,0} \leq g_{\min }$, then we can apply the procedure used in (GGB.i) to $\mathcal{T}\left[\vec{\rho}_{0}\right] \in \mathrm{GGB}$ and obtain that

$$
\mathcal{T}^{k}\left[\vec{\rho}_{0}\right]=\left(\hat{\rho}_{1}\left(f_{3,0}-f_{2}^{\max }\right), \rho_{2, c}, \rho_{3,0}\right) \in \mathrm{GGB}, \quad k \geq 1
$$

Since $Q\left(\vec{\rho}_{0}\right)=f_{3,0}=Q\left(\mathcal{T}\left[\vec{\rho}_{0}\right]\right)=Q\left(\mathcal{T}^{2}\left[\vec{\rho}_{0}\right]\right)$, we have that $Q^{l}=Q\left(\vec{\rho}_{0}\right)$ and therefore $\vec{\rho}^{l}=\mathcal{R}_{j}\left[\vec{\rho}_{0}\right]$. Hence $(6.8)$ holds because $\bar{\rho}_{h}^{l}=\tau_{h}=\tau_{h}^{3}, h \in \mathrm{H}$.

(BGB.iv-5) If $\alpha f_{3,0} \leq f_{3,0}-f_{2}^{\max }$ and $f_{3,0}>g_{\min }$, then $\alpha g_{\min }<\alpha f_{3,0} \leq f_{3,0}-f_{2}^{\max }<f_{1,0}<f_{1}^{\max }$, hence we can apply the procedure used in (GGB.ii) to $\mathcal{T}\left[\vec{\rho}_{0}\right] \in \mathrm{GGB}$, obtaining that $Q\left(\mathcal{T}\left[\vec{\rho}_{0}\right]\right)=g_{\text {min }}$ and

$$
\mathcal{T}^{k}\left[\vec{\rho}_{0}\right]=\left\{\begin{array}{ll}
\left(\hat{\rho}_{1}\left(\alpha g_{\min }\right), \hat{\rho}_{2}\left((1-\alpha) g_{\min }\right), \check{\rho}_{3}\left(g_{\min }\right)\right) \in \mathrm{GGG} & \text { if } g_{\min }-f_{2}^{\max }<\alpha g_{\min }<f_{1}^{\max }, \\
\left(\hat{\rho}_{1}\left(g_{\min }-f_{2}^{\max }\right), \rho_{2, c}, \check{\rho}_{3}\left(g_{\min }\right)\right) \in \mathrm{GGG} & \text { if } \alpha g_{\min } \leq g_{\min }-f_{2}^{\max },
\end{array} \quad k \geq 2 .\right.
$$

Since $Q\left(\vec{\rho}_{0}\right)=f_{3,0}>g_{\text {min }}=Q\left(\mathcal{T}\left[\vec{\rho}_{0}\right]\right)=Q\left(\mathcal{T}^{2}\left[\vec{\rho}_{0}\right]\right)$, we have that $Q^{l}=g_{\text {min }}$. Since $\alpha Q^{l}<\Delta_{1,0}$ and $Q^{l} \leq$ $Q\left(\vec{\rho}_{0}\right)<f_{1,0}+f_{2}^{\max }=\Delta_{1,0}+\Delta_{2,0}$, by $(6.9 \mathrm{c})$ and $(6.9 \mathrm{~d})$ we have

$$
\begin{cases}f_{1}\left(\bar{\rho}_{1}^{l}\right)=\alpha g_{\min }, \quad f_{2}\left(\bar{\rho}_{2}^{l}\right)=(1-\alpha) g_{\min }, \quad f_{3}\left(\bar{\rho}_{3}^{l}\right)=g_{\min } & \text { if } g_{\min }-\Delta_{2,0}<\alpha g_{\min }<\Delta_{1,0}, \\ f_{1}\left(\bar{\rho}_{1}^{l}\right)=g_{\min }-\Delta_{2,0}, \quad f_{2}\left(\bar{\rho}_{2}^{l}\right)=\Delta_{2,0}, \quad f_{3}\left(\bar{\rho}_{3}^{l}\right)=g_{\min } & \text { if } \alpha g_{\min } \leq g_{\min }-\Delta_{2,0}<\Delta_{1,0} .\end{cases}
$$

Hence (6.8) holds because $\bar{\rho}_{h}^{l}=\tau_{h}^{2}=\tau_{h}^{3}, h \in \mathrm{H}$.

(GBB) The case $\vec{\rho}_{0} \in \mathrm{GBB}$ is analogous to the case (BGB).

$(\mathrm{BBB})$ If $\vec{\rho}_{0} \in \mathrm{BBB}$, then $\Delta_{i, 0}=f_{i, 0}, i \in \mathrm{I}$, and $\Sigma_{3,0}=f_{3,0}$. Clearly

$$
Q\left(\vec{\rho}_{0}\right)=\min \left\{f_{3,0}, g\left(f_{1,0}+f_{2,0}\right)\right\} \leq g\left(f_{1,0}+f_{2,0}\right) .
$$

We distinguish the following cases:

(BBB.i) If $f_{3,0}=f_{1,0}+f_{2,0} \leq Q\left(\vec{\rho}_{0}\right)$, then $f_{3,0}=f_{1,0}+f_{2,0}=Q\left(\vec{\rho}_{0}\right)$ and $\Gamma_{i}\left(\vec{\rho}_{0}\right)=f_{i, 0}, i \in \mathrm{I}$, and therefore $\mathcal{T}\left[\vec{\rho}_{0}\right]=\vec{\rho}_{0}$, whence $\vec{\rho}_{0}$ is a fixed point for $\mathcal{T}$. Thus we have

$$
\mathcal{T}^{k}\left[\vec{\rho}_{0}\right]=\vec{\rho}_{0} \in \mathrm{BBB}, \quad k \geq 1 .
$$

Since $Q\left(\vec{\rho}_{0}\right)=Q\left(\mathcal{T}\left[\vec{\rho}_{0}\right]\right)=Q\left(\mathcal{T}^{2}\left[\vec{\rho}_{0}\right]\right)$, we have that $Q^{l}=Q\left(\vec{\rho}_{0}\right)$ and therefore $\vec{\rho}^{l}=\mathcal{R}_{j}\left[\vec{\rho}_{0}\right]$. Hence (6.8) holds because $\bar{\rho}_{h}^{l}=\tau_{h}=\tau_{h}^{3}, h \in \mathrm{H}$. Notice that this case corresponds to (6.9a).

(BBB.ii) If $f_{3,0} \neq f_{1,0}+f_{2,0} \leq Q\left(\vec{\rho}_{0}\right)$, then by Remark 2.10 we have $g\left(f_{1,0}+f_{2,0}\right)=f_{3}^{\max }$ and $Q\left(\vec{\rho}_{0}\right)=f_{3,0}$. Moreover $\Gamma_{i}\left(\vec{\rho}_{0}\right)=f_{i, 0}, i \in \mathrm{I}$, and therefore

$$
\mathcal{T}\left[\vec{\rho}_{0}\right]=\left(\rho_{1,0}, \rho_{2,0}, \check{\rho}_{3}\left(f_{1,0}+f_{2,0}\right)\right) \in \mathrm{BBG} .
$$

We observe that $f_{3}^{\max }=Q\left(\mathcal{T}\left[\vec{\rho}_{0}\right]\right)>Q\left(\vec{\rho}_{0}\right)=f_{3,0}>f_{1,0}+f_{2,0}$. We can then apply the procedure used in (BBG.i) to $\mathcal{T}\left[\vec{\rho}_{0}\right] \in \mathrm{BBG}$ and obtain that

$$
\mathcal{T}^{k}\left[\vec{\rho}_{0}\right]=\left(\rho_{1,0}, \rho_{2,0}, \check{\rho}_{3}\left(f_{1,0}+f_{2,0}\right)\right) \in \mathrm{BBG}, \quad k \geq 1 .
$$

Since $Q\left(\vec{\rho}_{0}\right)=f_{3,0} \leq f_{3}^{\max }=Q\left(\mathcal{T}\left[\vec{\rho}_{0}\right]\right)=Q\left(\mathcal{T}^{2}\left[\vec{\rho}_{0}\right]\right)$, we have that $Q^{l}=Q\left(\vec{\rho}_{0}\right)$ and therefore $\vec{\rho}^{l}=\mathcal{R}_{j}\left[\vec{\rho}_{0}\right]$. Hence (6.8) holds because $\bar{\rho}_{h}^{l}=\tau_{h}=\tau_{h}^{3}, h \in \mathrm{H}$. Notice that this case corresponds to (6.9a). 
(BBB.iii) If $f_{1,0}+f_{2,0}>Q\left(\vec{\rho}_{0}\right)=g\left(f_{1,0}+f_{2,0}\right) \neq f_{3,0}$, then $f_{1,0}+f_{2,0}>f_{3}^{\max }$. Moreover

$$
\Gamma_{1}\left(\vec{\rho}_{0}\right)=\left\{\begin{array}{ll}
f_{1,0} & \text { if } \alpha Q\left(\vec{\rho}_{0}\right) \geq f_{1,0}, \\
\alpha Q\left(\vec{\rho}_{0}\right) & \text { if } Q\left(\vec{\rho}_{0}\right)-f_{2,0}<\alpha Q\left(\vec{\rho}_{0}\right)<f_{1,0}, \\
Q\left(\vec{\rho}_{0}\right)-f_{2,0} & \text { if } \alpha Q\left(\vec{\rho}_{0}\right) \leq Q\left(\vec{\rho}_{0}\right)-f_{2,0},
\end{array} \quad \Gamma_{2}\left(\vec{\rho}_{0}\right)=Q\left(\vec{\rho}_{0}\right)-\Gamma_{1}\left(\vec{\rho}_{0}\right),\right.
$$

and

$$
\mathcal{T}\left[\vec{\rho}_{0}\right]= \begin{cases}\left(\rho_{1,0}, \hat{\rho}_{2}\left(Q\left(\vec{\rho}_{0}\right)-f_{1,0}\right), \check{\rho}_{3}\left(Q\left(\vec{\rho}_{0}\right)\right)\right) \in \mathrm{BGG} & \text { if } \alpha Q\left(\vec{\rho}_{0}\right) \geq f_{1,0}, \\ \left(\hat{\rho}_{1}\left(\alpha Q\left(\vec{\rho}_{0}\right)\right), \hat{\rho}_{2}\left((1-\alpha) Q\left(\vec{\rho}_{0}\right)\right), \check{\rho}_{3}\left(Q\left(\vec{\rho}_{0}\right)\right)\right) \in \mathrm{GGG} & \text { if } Q\left(\vec{\rho}_{0}\right)-f_{2,0}<\alpha Q\left(\vec{\rho}_{0}\right)<f_{1,0}, \\ \left(\hat{\rho}_{1}\left(Q\left(\vec{\rho}_{0}\right)-f_{2,0}\right), \rho_{2,0}, \check{\rho}_{3}\left(Q\left(\vec{\rho}_{0}\right)\right)\right) \in \mathrm{GBG} & \text { if } \alpha Q\left(\vec{\rho}_{0}\right) \leq Q\left(\vec{\rho}_{0}\right)-f_{2,0} .\end{cases}
$$

Notice that $Q\left(\mathcal{T}\left[\vec{\rho}_{0}\right]\right) \leq Q\left(\vec{\rho}_{0}\right)$.

(BBB.iii-1) If $\alpha Q\left(\vec{\rho}_{0}\right) \geq \alpha g\left(f_{1,0}+f_{2}^{\max }\right) \geq f_{1,0}$, then $\alpha Q\left(\mathcal{T}\left[\vec{\rho}_{0}\right]\right)=\alpha g\left(f_{1,0}+f_{2}^{\max }\right) \geq f_{1,0}>f_{3}^{\max }-f_{2,0}>$ $Q\left(\mathcal{T}\left[\vec{\rho}_{0}\right]\right)-f_{2,0}$ and we can apply the procedure used in (BGG.i-1) to $\mathcal{T}\left[\vec{\rho}_{0}\right] \in$ BGG and obtain that

$$
\mathcal{T}^{k}\left[\vec{\rho}_{0}\right]=\left(\rho_{1,0}, \hat{\rho}_{2}\left(g\left(f_{1,0}+f_{2}^{\max }\right)-f_{1,0}\right), \check{\rho}_{3}\left(g\left(f_{1,0}+f_{2}^{\max }\right)\right)\right) \in \mathrm{BGG}, \quad k \geq 2 .
$$

Since $Q\left(\vec{\rho}_{0}\right) \geq Q\left(\mathcal{T}\left[\vec{\rho}_{0}\right]\right)=Q\left(\mathcal{T}^{2}\left[\vec{\rho}_{0}\right]\right)$, we have that $Q^{l}=Q\left(\mathcal{T}\left[\vec{\rho}_{0}\right]\right)=g\left(f_{1,0}+f_{2}^{\max }\right)$. Since $Q^{l}-\Delta_{2,0}<\Delta_{1,0} \leq$ $\alpha Q^{l}$, by $(6.9 \mathrm{~b})$

$$
f_{1}\left(\bar{\rho}_{1}^{l}\right)=f_{1,0}, \quad f_{2}\left(\bar{\rho}_{2}^{l}\right)=g\left(f_{1,0}+f_{2}^{\max }\right)-f_{1,0}, \quad f_{3}\left(\bar{\rho}_{3}^{l}\right)=g\left(f_{1,0}+f_{2}^{\max }\right) .
$$

Hence (6.8) holds because $\bar{\rho}_{h}^{l}=\tau_{h}^{2}=\tau_{h}^{3}, h \in \mathrm{H}$.

(BBB.iii-2) If $\alpha Q\left(\vec{\rho}_{0}\right) \geq f_{1,0}>\alpha g\left(f_{1,0}+f_{2}^{\max }\right)$, then $\alpha g_{\min } \leq \alpha Q\left(\mathcal{T}\left[\vec{\rho}_{0}\right]\right)=\alpha g\left(f_{1,0}+f_{2}^{\max }\right)<f_{1,0}$ and $(1-\alpha) g_{\min } \leq(1-\alpha) Q\left(\mathcal{T}\left[\vec{\rho}_{0}\right]\right) \leq(1-\alpha) Q\left(\vec{\rho}_{0}\right) \leq Q\left(\vec{\rho}_{0}\right)-f_{1,0}<f_{2,0}<f_{2}^{\max }$. Therefore we can apply the procedure used in (BGG.i-2) to $\mathcal{T}\left[\vec{\rho}_{0}\right] \in$ BGG and obtain that

$$
\begin{array}{ll}
\mathcal{T}^{2}\left[\vec{\rho}_{0}\right]=\left(\hat{\rho}_{1}\left(\alpha Q\left(\mathcal{T}\left[\vec{\rho}_{0}\right]\right)\right), \hat{\rho}_{2}\left((1-\alpha) Q\left(\mathcal{T}\left[\vec{\rho}_{0}\right]\right)\right), \check{\rho}_{3}\left(Q\left(\mathcal{T}\left[\vec{\rho}_{0}\right]\right)\right)\right) \in \mathrm{GGG}, & \\
\mathcal{T}^{k}\left[\vec{\rho}_{0}\right]=\left(\hat{\rho}_{1}\left(\alpha g_{\min }\right), \hat{\rho}_{2}\left((1-\alpha) g_{\min }\right), \check{\rho}_{3}\left(g_{\min }\right)\right) \in \mathrm{GGG}, & k \geq 3 .
\end{array}
$$

Since $Q\left(\vec{\rho}_{0}\right)=g\left(f_{1,0}+f_{2,0}\right) \geq g\left(f_{1,0}+f_{2}^{\max }\right)=Q\left(\mathcal{T}\left[\vec{\rho}_{0}\right]\right) \geq Q\left(\mathcal{T}^{2}\left[\vec{\rho}_{0}\right]\right)=g_{\min }$, we have that $Q^{l}=g_{\min }$. Since $Q^{l}-\Delta_{2,0}<\alpha Q^{l}<\Delta_{1,0}$, by $(6.9 \mathrm{c})$

$$
f_{1}\left(\bar{\rho}_{1}^{l}\right)=\alpha g_{\min }, \quad f_{2}\left(\bar{\rho}_{2}^{l}\right)=(1-\alpha) g_{\min }, \quad f_{3}\left(\bar{\rho}_{3}^{l}\right)=g_{\min } .
$$

Hence (6.8) holds because $\bar{\rho}_{h}^{l}=\tau_{h}^{3}, h \in \mathrm{H}$.

(BBB.iii-3) If $Q\left(\vec{\rho}_{0}\right)-f_{2,0}<\alpha Q\left(\vec{\rho}_{0}\right)<f_{1,0}$, then $Q\left(\mathcal{T}\left[\vec{\rho}_{0}\right]\right)=g_{\min }$ and $g_{\min }-f_{2}^{\max }<g_{\min }-f_{2,0}<\alpha g_{\min }<$ $f_{1,0}<f_{1}^{\max }$. Therefore we can apply the procedure used in (GGG) to $\mathcal{T}\left[\vec{\rho}_{0}\right] \in \mathrm{GGG}$ and obtain that

$$
\mathcal{T}^{k}\left[\vec{\rho}_{0}\right]=\left(\hat{\rho}_{1}\left(\alpha g_{\min }\right), \hat{\rho}_{2}\left((1-\alpha) g_{\min }\right), \check{\rho}_{3}\left(g_{\min }\right)\right) \in \mathrm{GGG}, \quad k \geq 2 .
$$

Since $Q\left(\vec{\rho}_{0}\right)=g\left(f_{1,0}+f_{2,0}\right) \geq Q\left(\mathcal{T}\left[\vec{\rho}_{0}\right]\right)=Q\left(\mathcal{T}^{2}\left[\vec{\rho}_{0}\right]\right)=g_{\text {min }}$, we have that $Q^{l}=g_{\text {min }}$. Since $Q^{l}-\Delta_{2,0}<\alpha Q^{l}<$ $\Delta_{1,0}$, by $(6.9 \mathrm{c})$

$$
f_{1}\left(\bar{\rho}_{1}^{l}\right)=\alpha g_{\min }, \quad f_{2}\left(\bar{\rho}_{2}^{l}\right)=(1-\alpha) g_{\min }, \quad f_{3}\left(\bar{\rho}_{3}^{l}\right)=g_{\min } .
$$

Hence (6.8) holds because $\bar{\rho}_{h}^{l}=\tau_{h}^{2}=\tau_{h}^{3}, h \in \mathrm{H}$.

(BBB.iii-4) The case $\alpha Q\left(\vec{\rho}_{0}\right) \leq Q\left(\vec{\rho}_{0}\right)-f_{2,0}$ can be treated either as in the case (BBB.iii-1), in this case we refer to (BBB.iii-4.a), or as in (BBB.iii-2), we call this case (BBB-iii-4.b). 
(BBB.iv) If $f_{1,0}+f_{2,0}>Q\left(\vec{\rho}_{0}\right)=f_{3,0}$, then

$$
\Gamma_{1}\left(\vec{\rho}_{0}\right)=\left\{\begin{array}{ll}
f_{1,0} & \text { if } \alpha f_{3,0} \geq f_{1,0}, \\
\alpha f_{3,0} & \text { if } f_{3,0}-f_{2,0}<\alpha f_{3,0}<f_{1,0}, \\
f_{3,0}-f_{2,0} & \text { if } \alpha f_{3,0} \leq f_{3,0}-f_{2,0},
\end{array} \quad \Gamma_{2}\left(\vec{\rho}_{0}\right)=f_{3,0}-\Gamma_{1}\left(\vec{\rho}_{0}\right),\right.
$$

and

$$
\mathcal{T}\left[\vec{\rho}_{0}\right]= \begin{cases}\left(\rho_{1,0}, \hat{\rho}_{2}\left(f_{3,0}-f_{1,0}\right), \rho_{3,0}\right) \in \mathrm{BGB} & \text { if } \alpha f_{3,0} \geq f_{1,0}, \\ \left(\hat{\rho}_{1}\left(\alpha f_{3,0}\right), \hat{\rho}_{2}\left((1-\alpha) f_{3,0}\right), \rho_{3,0}\right) \in \mathrm{GGB} & \text { if } f_{3,0}-f_{2,0}<\alpha f_{3,0}<f_{1,0}, \\ \left(\hat{\rho}_{1}\left(f_{3,0}-f_{2,0}\right), \rho_{2,0}, \rho_{3,0}\right) \in \mathrm{GBB} & \text { if } \alpha f_{3,0} \leq f_{3,0}-f_{2,0} .\end{cases}
$$

Notice that $Q\left(\mathcal{T}\left[\vec{\rho}_{0}\right]\right) \leq Q\left(\vec{\rho}_{0}\right)=f_{3,0}<f_{1,0}+f_{2}^{\max }$ and $f_{1,0}+f_{2,0}>Q\left(\vec{\rho}_{0}\right) \geq Q\left(\mathcal{T}\left[\vec{\rho}_{0}\right]\right)$.

(BBB.iv-1) If $\alpha f_{3,0}>\alpha g\left(f_{1,0}+f_{2}^{\max }\right) \geq f_{1,0}$, then $Q\left(\mathcal{T}\left[\vec{\rho}_{0}\right]\right) \neq f_{3,0}$ and $\alpha Q\left(\vec{\rho}_{0}\right)>\alpha Q\left(\mathcal{T}\left[\vec{\rho}_{0}\right]\right) \geq f_{1,0}$, hence by applying the procedure used in (BGB.iii-1) to $\mathcal{T}\left[\vec{\rho}_{0}\right] \in$ BGB we obtain that

$$
\mathcal{T}^{k}\left[\vec{\rho}_{0}\right]=\left(\rho_{1,0}, \hat{\rho}_{2}\left(Q\left(\mathcal{T}\left[\vec{\rho}_{0}\right]\right)-f_{1,0}\right), \check{\rho}_{3}\left(Q\left(\mathcal{T}\left[\vec{\rho}_{0}\right]\right)\right)\right) \in \mathrm{BGG}, \quad k \geq 2 .
$$

Since $Q\left(\vec{\rho}_{0}\right)=f_{3,0}>g\left(f_{1,0}+f_{2}^{\max }\right)=Q\left(\mathcal{T}\left[\vec{\rho}_{0}\right]\right)=Q\left(\mathcal{T}^{2}\left[\vec{\rho}_{0}\right]\right)$, we have that $Q^{l}=Q\left(\mathcal{T}\left[\vec{\rho}_{0}\right]\right)$. Since $\Delta_{1,0}+\Delta_{2,0}>$ $Q^{l}$ and $\alpha Q^{l} \geq \Delta_{1,0}$, by $(6.9 \mathrm{~b})$

$$
f_{1}\left(\bar{\rho}_{1}^{l}\right)=f_{1,0}, \quad f_{2}\left(\bar{\rho}_{2}^{l}\right)=Q\left(\mathcal{T}\left[\vec{\rho}_{0}\right]\right)-f_{1,0}, \quad f_{3}\left(\bar{\rho}_{3}^{l}\right)=Q\left(\mathcal{T}\left[\vec{\rho}_{0}\right]\right) .
$$

Hence (6.8) holds because $\bar{\rho}_{h}^{l}=\tau_{h}^{2}=\tau_{h}^{3}, h \in \mathrm{H}$.

(BBB.iv-2) If $\alpha f_{3,0} \geq f_{1,0}>\alpha g\left(f_{1,0}+f_{2}^{\max }\right)$, then $(1-\alpha) Q\left(\mathcal{T}\left[\vec{\rho}_{0}\right]\right)<(1-\alpha) Q\left(\vec{\rho}_{0}\right) \leq Q\left(\vec{\rho}_{0}\right)-f_{1,0}<f_{2,0}<$ $f_{2}^{\max }$ and $\alpha Q\left(\mathcal{T}\left[\vec{\rho}_{0}\right]\right)<f_{1,0}$, hence by applying the procedure used in (BGB.iii-2) to $\mathcal{T}\left[\vec{\rho}_{0}\right] \in$ BGB we obtain

$$
\begin{array}{ll}
\mathcal{T}^{2}\left[\vec{\rho}_{0}\right]=\left(\hat{\rho}_{1}\left(\alpha Q\left(\mathcal{T}\left[\vec{\rho}_{0}\right]\right)\right), \hat{\rho}_{2}\left((1-\alpha) Q\left(\mathcal{T}\left[\vec{\rho}_{0}\right]\right)\right), \check{\rho}_{3}\left(Q\left(\mathcal{T}\left[\vec{\rho}_{0}\right]\right)\right)\right) \in \mathrm{GGG}, & \\
\mathcal{T}^{k}\left[\vec{\rho}_{0}\right]=\left(\hat{\rho}_{1}\left(\alpha g_{\min }\right), \hat{\rho}_{2}\left((1-\alpha) g_{\min }\right), \check{\rho}_{3}\left(g_{\min }\right)\right) \in \mathrm{GGG}, & k \geq 3 .
\end{array}
$$

Since $Q\left(\vec{\rho}_{0}\right)=f_{3,0}>Q\left(\mathcal{T}\left[\vec{\rho}_{0}\right]\right)=g\left(f_{1,0}+f_{2}^{\max }\right) \geq Q\left(\mathcal{T}^{2}\left[\vec{\rho}_{0}\right]\right)=g_{\text {min }}$, we have that $Q^{l}=g_{\text {min }}$. Since $Q^{l}-\Delta_{2,0}<$ $\alpha Q^{l}<\Delta_{1,0}$, by $(6.9 \mathrm{c})$

$$
f_{1}\left(\bar{\rho}_{1}^{l}\right)=\alpha g_{\min }, \quad f_{2}\left(\bar{\rho}_{2}^{l}\right)=(1-\alpha) g_{\min }, \quad f_{3}\left(\bar{\rho}_{3}^{l}\right)=g_{\min } .
$$

Hence (6.8) holds because $\bar{\rho}_{h}^{l}=\tau_{h}^{3}, h \in \mathrm{H}$.

(BBB.iv-3) If $\alpha g\left(f_{1,0}+f_{2}^{\max }\right) \geq \alpha f_{3,0} \geq f_{1,0}$, then $\alpha Q\left(\vec{\rho}_{0}\right)=\alpha Q\left(\mathcal{T}\left[\vec{\rho}_{0}\right]\right)=\alpha f_{3,0} \geq f_{1,0}$ and we can apply the procedure used in (BGB.iv-1) to $\mathcal{T}\left[\vec{\rho}_{0}\right] \in \mathrm{BGB}$ and obtain that

$$
\mathcal{T}^{k}\left[\vec{\rho}_{0}\right]=\left(\rho_{1,0}, \hat{\rho}_{2}\left(f_{3,0}-f_{1,0}\right), \rho_{3,0}\right) \in \mathrm{BGB}, \quad k \geq 1 .
$$

Since $Q\left(\vec{\rho}_{0}\right)=Q\left(\mathcal{T}\left[\vec{\rho}_{0}\right]\right)=Q\left(\mathcal{T}^{2}\left[\vec{\rho}_{0}\right]\right)$, we have that $Q^{l}=Q\left(\vec{\rho}_{0}\right)$ and therefore $\vec{\rho}^{l}=\mathcal{R}_{j}\left[\vec{\rho}_{0}\right]$. Hence (6.8) holds because $\bar{\rho}_{h}^{l}=\tau_{h}=\tau_{h}^{3}, h \in \mathrm{H}$.

(BBB.iv-4) If $Q\left(\vec{\rho}_{0}\right)-f_{2,0}<\alpha Q\left(\vec{\rho}_{0}\right)<f_{1,0}$ and $f_{3,0} \leq g_{\min }$, then $f_{3,0}-f_{2}^{\max }<\alpha f_{3,0}<f_{1}^{\max }$, therefore we can apply the procedure used in (GGB.i) to $\mathcal{T}\left[\vec{\rho}_{0}\right] \in$ GGB and obtain that

$$
\mathcal{T}^{k}\left[\vec{\rho}_{0}\right]=\left(\hat{\rho}_{1}\left(\alpha f_{3,0}\right), \hat{\rho}_{2}\left((1-\alpha) f_{3,0}\right), \rho_{3,0}\right) \in \mathrm{GGB}, \quad k \geq 1 .
$$

Since $Q\left(\vec{\rho}_{0}\right)=Q\left(\mathcal{T}\left[\vec{\rho}_{0}\right]\right)=Q\left(\mathcal{T}^{2}\left[\vec{\rho}_{0}\right]\right)=f_{3,0}$, we have that $Q^{l}=Q\left(\vec{\rho}_{0}\right)$ and therefore $\vec{\rho}^{l}=\mathcal{R}_{j}\left[\vec{\rho}_{0}\right]$. Hence $(6.8)$ holds because $\bar{\rho}_{h}^{l}=\tau_{h}=\tau_{h}^{3}, h \in \mathrm{H}$.

(BBB.iv-5) If $Q\left(\vec{\rho}_{0}\right)-f_{2,0}<\alpha Q\left(\vec{\rho}_{0}\right)<f_{1,0}$ and $f_{3,0}>g_{\min }$, then $g_{\min }-f_{2}^{\max }<g_{\min }-f_{2,0}<\alpha g_{\min }<f_{1,0}<$ $f_{1}^{\max }$, therefore we can apply the procedure used in (GGB.ii) to $\mathcal{T}\left[\vec{\rho}_{0}\right] \in \mathrm{GGB}$ and obtain that

$$
\mathcal{T}^{k}\left[\vec{\rho}_{0}\right]=\left(\hat{\rho}_{1}\left(\alpha g_{\min }\right), \hat{\rho}_{2}\left((1-\alpha) g_{\min }\right), \check{\rho}_{3}\left(g_{\min }\right)\right) \in \mathrm{GGG}, \quad k \geq 2 .
$$


Since $Q\left(\vec{\rho}_{0}\right) \geq Q\left(\mathcal{T}\left[\vec{\rho}_{0}\right]\right)=Q\left(\mathcal{T}^{2}\left[\vec{\rho}_{0}\right]\right)=g_{\text {min }}$, we have that $Q^{l}=g_{\text {min }}$. Since $Q^{l}-\Delta_{2,0}<\alpha Q^{l}<\Delta_{1,0}$, by $(6.9 \mathrm{c})$

$$
f_{1}\left(\bar{\rho}_{1}^{l}\right)=\alpha g_{\min }, \quad f_{2}\left(\bar{\rho}_{2}^{l}\right)=(1-\alpha) g_{\min }, \quad f_{3}\left(\bar{\rho}_{3}^{l}\right)=g_{\min } .
$$

Hence (6.8) holds because $\bar{\rho}_{h}^{l}=\tau_{h}^{2}=\tau_{h}^{3}, h \in \mathrm{H}$.

(BBB.iv-6) The case $\alpha f_{3,0} \leq f_{3,0}-f_{2,0}$ can be treated either as in case (BBB.iv-1) or (BBB.iv-2) or (BBB.iv-3). We refer to these cases as (BBB.iv-6.a), (BBB.iv-6.b) and (BBB.iv-6.c), respectively.

Finally, the above case by case analysis proves also that for any $\vec{\rho}_{0} \in \Lambda$ we have $\mathcal{T}^{k}\left[\vec{\rho}_{0}\right]=\mathcal{T}^{3}\left[\vec{\rho}_{0}\right]$ for any $k \geq 4$.

Finally, Proposition 3.1 follows from the above case by case analysis.

\section{Conclusions And Perspectives}

The numerical simulations in this paper show that the first order traffic model subject to a non-local point constraint at the junction reproduces in a realistic way the capacity drop phenomenon at a road merge. Moreover, our finite volumes numerical scheme converges numerically to the exact solution. In order to further our investigation to the study of well-posedness of the general Cauchy problem associated to the model and to obtain a mathematical convergence proof of the numerical scheme, we need to complete the analysis of the Cauchy problem at a merge subject to a constant constraint on the receiving capacity at the junction. This work is in preparation.

Acknowledgements. The first three authors acknowledge the support of the Région Bourgogne Franche-Comté, projet OPE-2017-0067. "Analyse mathématique et simulation numérique d'EDP issus de problèmes de contrôle et du trafic routier" and of the Université de Bourgogne Franche-Comté, projet Chrysalide 2017 "Contrôle, analyse numérique et applications d'équations hyperboliques sur un réseau". The present research was supported by the European Commission under the project STREVCOMS PIRSES-2013-612669, Università degli Studi di Ferrara Project 2017 "FIR: Modelli macroscopici per il traffico veicolare o pedonale" and by the National Science Centre, Poland, Project "Mathematics of multi-scale approaches in life and social sciences" No. 2017/25/B/ST1/00051. EDS, SFP and MDR are members of the Gruppo Nazionale per l'Analisi Matematica, la Probabilità e le loro Applicazioni (GNAMPA) of the Istituto Nazionale di Alta Matematica (INdAM).

\section{REFERENCES}

[1] B. Andreianov, G.M. Coclite and C. Donadello, Well-posedness for vanishing viscosity solutions of scalar conservation laws on a network. Discrete Contin. Dyn. Syst. A 37 (2017) 5913-5942.

[2] B. Andreianov, C. Donadello, U. Razafison and M.D. Rosini, Riemann problems with non-local point constraints and capacity drop. Math. Biosci. Eng. 12 (2015) 259-278.

[3] B. Andreianov, C. Donadello, U. Razafison and M.D. Rosini, Qualitative behaviour and numerical approximation of solutions to conservation laws with non-local point constraints on the flux and modeling of crowd dynamics at the bottlenecks. ESAIM: M2AN 50 (2016) 1269-1287.

[4] B. Andreianov, C. Donadello, U. Razafison and M.D. Rosini, Analysis and approximation of one-dimensional scalar conservation laws with general point constraints on the flux. J. Math. Pures App. 116 (2018) 309-346.

[5] B. Andreianov, C. Donadello and M.D. Rosini, Crowd dynamics and conservation laws with nonlocal constraints and capacity drop. Math. Models Methods Appl. Sci. 24 (2014) 2685-2722.

[6] B. Andreianov, P. Goatin and N. Seguin, Finite volume schemes for locally constrained conservation laws. Numer. Math. 115 (2010) 609-645.

[7] C. Bardos, A.Y. Leroux and J.C. Nedelec, First order quasilinear equations with boundary conditions. Commun. Partial Differ. Equ. 4 (1979) 1017-1034.

[8] A. Bressan, S. Čanić, M. Garavello, M. Herty and B. Piccoli, Flows on networks: recent results and perspectives. EMS Surv. Math. Sci. 1 (2014) 47-111.

[9] C. Cancès and N. Seguin, Error estimate for Godunov approximation of locally constrained conservation laws. SIAM J. Numer. Anal. 50 (2012) 3036-3060.

[10] C. Chalons, Numerical approximation of a macroscopic model of pedestrian flows. SIAM J. Sci. Comput. 29 (2007) 539-555.

[11] C. Chalons, P. Goatin and N. Seguin, General constrained conservation laws. Application to pedestrian flow modeling. Networks Heterogen. Media 8 (2013) 433-463. 
[12] G.M. Coclite, M. Garavello and B. Piccoli, Traffic flow on a road network. SIAM J. Math. Anal. 36 (2005) $1862-1886$.

[13] R. Colombo and P. Goatin, A well posed conservation law with a variable unilateral constraint. J. Differ. Equ. 234 (2007) 654-675.

[14] R.M. Colombo, P. Goatin and M.D. Rosini, A macroscopic model for pedestrian flows in panic situations. In: Current Advances in Nonlinear Analysis and Related Topics. GAKUTO Internat. Ser. Math. Sci. Appl. Gakkōtosho, Tokyo 32 (2010) $255-272$.

[15] R.M. Colombo, P. Goatin and M.D. Rosini, On the modelling and management of traffic. ESAIM: M2AN 45 (2011) 853-872.

[16] R.M. Colombo and M.D. Rosini, Pedestrian flows and non-classical shocks. Math. Methods Appl. Sci. 28 (2005) $1553-1567$.

[17] R.M. Colombo and M.D. Rosini, Existence of nonclassical solutions in a pedestrian flow model. Nonlinear Anal.: Real World App. 10 (2009) 2716-2728.

[18] C. D'Apice, R. Manzo and B. Piccoli, Packet flow on telecommunication networks. SIAM J. Math. Anal. 38 (2006) 717-740.

[19] M. Garavello, K. Han and B. Piccoli, Models for vehicular traffic on networks. Conservation laws models. AIMS Series on Applied Mathematics. American Institute of Mathematical Sciences (AIMS), Springfield, MO 9 (2016).

[20] M. Garavello and B. Piccoli, Traffic flow on networks. Conservation laws models. AIMS Series on Applied Mathematics. American Institute of Mathematical Sciences (AIMS), Springfield, MO 1 (2006).

[21] M. Garavello and B. Piccoli, Conservation laws on complex networks. Ann. Inst. Henri Poincaré (C) Non Lin. Anal. 26 (2009) $1925-1951$.

[22] B. Haut, G. Bastin and Y. Chitour, A macroscopic traffic model for road networks with a representation of the capacity drop phenomenon at the junctions. In Vol. 226 of Proceedings 16th IFAC World Congress, Prague, Czech Republic (2005) $\mathrm{Tu}-\mathrm{M} 01-\mathrm{TP} / 3$.

[23] S.N. Kruzhkov, First order quasilinear equations with several independent variables. Mat. Sb. (N.S.) 81 (1970) $228-255$.

[24] M.J. Lighthill and G.B. Whitham On kinematic waves. II. A theory of traffic flow on long crowded roads. Proc. Roy. Soc. London. Ser. A. 229 (1955) 317-345.

[25] E.Y. Panov, Existence of strong traces for quasi-solutions of multidimensional conservation laws. J. Hyperbolic Differ. Equ. 4 (2007) 729-770.

[26] S.F. Pellegrino, On the implementation of a finite volumes scheme with monotone transmission conditions for scalar conservation laws on a star-shaped network. Preprint arXiv:1902.02395 (2019).

[27] P.I. Richards, Shock waves on the highway. Oper. Res. 4 (1956) 42-51.

[28] M.D. Rosini, Macroscopic Models for Vehicular Flows and Crowd Dynamics: Theory and Applications. Springer, Heidelberg (2013).

[29] A. Vasseur, Strong traces for solutions of multidimensional scalar conservation laws. Arch. Ration. Mech. Anal. 160 (2001) 181-193. 